Centro

deInformática

Pós-Graduação em Ciência da Computação

"Associating Genotype Sequence Properties to Haplotype Inference Errors"

by

Rogério dos Santos Rosa

Ph.D. Thesis

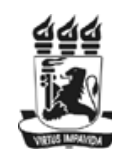

Universidade Federal de Pernambuco

posgraduacao@cin.ufpe.br

www.cin.ufpe.br/ posgraduacao

RECIFE/2015 


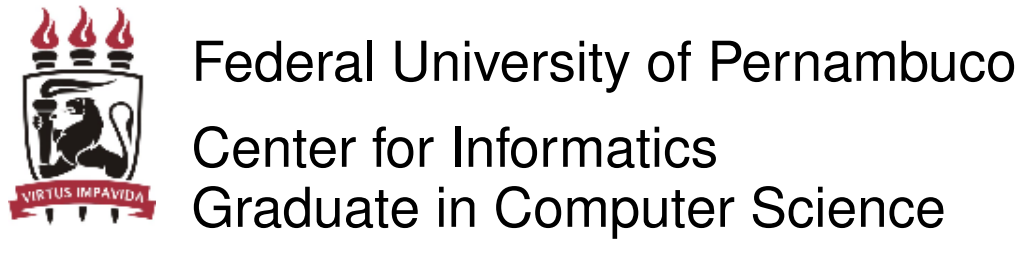

Rogério dos Santos Rosa

\section{ASSOCIATING GENOTYPE SEQUENCE PROPERTIES TO HAPLOTYPE INFERENCE ERRORS}

A Ph.D. Thesis presented to the Center for Informatics of Federal University of Pernambuco in partial fulfillment of the requirements for the degree of Philosophy Doctor in Computer Science. 
Catalogação na fonte

Bibliotecária Jane Souto Maior, CRB4-571

\section{R788a Rosa, Rogério dos Santos}

Associating genotype sequence properties to haplotype inference errors / Rogério dos Santos Rosa. - Recife: O Autor, 2015.

108 f.: il. fig., tab.

Orientador: Katia Silva Guimarães.

Tese (Doutorado) - Universidade Federal de Pernambuco. Cln, Ciência da computação, 2015.

Inclui referências.

1. Ciência da computação. 2. Programas estatísticos. I. Guimarães, Katia Silva (orientador). II. Título.

$004 \quad$ CDD (23. ed.) UFPE- MEI 2015-144 
Tese de Doutorado apresentada por Rogério dos Santos Rosa à Pós-Graduação em Ciência da Computação do Centro de Informática da Universidade Federal de Pernambuco, sob o título “Associating Genotype Sequence Properties to Haplotype Inference Errors", orientada pela Profa. Katia Silva Guimarães e aprovada pela Banca Examinadora formada pelos professores:

Prof. Silvio de Barros Melo

Centro de Informática / UFPE

Profa. Renata Cardoso Rodrigues de Souza

Centro de Informática / UFPE

Prof. George Darmiton da Cunha Cavalcanti

Centro de Informática / UFPE

Profa. Ana Maria Benko Iseppon

Departamento de Genética / UFPE

Prof. Alberto Martín Rivera Dávila

Laboratório de Biologia Computacional e Sistemas /

FIOCRUZ

Prof. Leandro Chaves Rêgo

Departamento de Estatística / UFPE

Visto e permitida a impressão.

Recife, 12 de março de 2015.

\section{Profa. Edna Natividade da Silva Barros}

Coordenador da Pós-Graduação em Ciência da Computação do

Centro de Informática da Universidade Federal de Pernambuco. 
I dedicate this thesis to all my family, friends and professors who gave me the necessary support to get here. 


\section{Acknowledgements}

I would like to thank all my teachers from the Instituto Estadual de Educação Guilherme Clemente Koehler (Ijuí/RS) for the excellent public education that I received there, and for having awakened in me the scientific curiosity and critical thinking. These were crucial for me to get here. Special thanks to teacher Astrid Schmidt (in memoriam) for the great teachings and life example.

For my teachers from Colégio Sagrado Coração de Jesus (Ijuí/RS), where I had my first contact with Informatics in the technical course, my special thanks. First as masters, and after as colleagues and friends, the teachers Julio Cesar Beal Junior and Bruno Batista Boniati were my examples and inspiration. I would like to thank my colleagues that worked with me in the Coordenadoria de Informática (UNIJUÍ), that was a very special moment in my life, and I learned a lot with them.

My sincere thanks to all professors of the Informatics Center (UFPE), specially Liliane Salgado and Renata Souza, who on numerous occasions heard me and advised me in difficult moments.

My thanks and my affectionate tribute to Katia Silva Guimarães. Besides being my professor and advisor in the Masters and Doctoral programs, she was a very special friend. Thank you for your direct responsibility and cumplicity in the construction of this thesis, and for numerous advices and teachings that will take for the rest of my life.

Finally, I thank the financial support received from FACEPE for the development of this thesis (process no. PBPG-0070-1.03/10). 
When I was five years old, my mother always told me that happiness was the key to life. When I went to school, they asked me what I wanted to be when I grew up. I wrote down 'happy'. They told me I didn't understand the assignment, and I told them they didn't understand life. 


\section{Resumo}

Haplótipos têm um papel central na compreensão e diagnóstico de determinadas doenças e também para estudos de evolução. Este tipo de informação é difícil de obter diretamente, diante disto, métodos computacionais para inferir haplótipos a partir de dados genotípicos têm recebido grande atenção da comunidade de biologia computacional. Infelizmente, a Inferência de Halótipos é um problema difícil e os métodos existentes só podem predizer parcialmente soluções corretas. Foram desenvolvidos modelos de redes neurais que utilizam diferentes propriedades dos dados para prever quando um método é mais propenso a cometer erros. Foram calibrados modelos para três abordagens de Inferência de Haplótipos diferentes e os resultados validados estatisticamente. Os resultados dos experimentos oferecem informações valiosas sobre o desempenho e comportamento desses métodos, gerando condições para o desenvolvimento de estratégias de combinação de diferentes soluções ou melhoria das abordagens individuais. Foi demonstrado que Desequilíbrio de Ligação (LD) e heterozigosidade são fortes indicadores de tendência de erro, desta forma foram delineados cenários com base em medidas de LD, que revelam quando um método tem maior ou menor propensão de cometer erros. Foi identificado que utilizando janelas de 10 SNPs (polimorfismo de um único nucleotídeo), imediatamente a montante, e eliminando os SNPs não informativos pelo Teste de Fisher leva-se a uma correlação mais adequada entre LD e a ocorrência de erros. Por fim, foi aplicada análise de Regressão Linear para explorar a relevância de várias propriedades biologicamente significativas das sequências de genótipos para a precisão dos resultados de Inferência de Haplótipos, estimou-se modelos para duas bases de dados (considerando apenas humanos) utilizando duas métricas de erro. A precisão dos resultados e a estabilidade dos modelos propostos foram validadas por testes estatísticos.

Palavras-chave: Regressão Linear. Análises Estatística. SNPs. Haplótipos. Dados Genotípicos. Inferência de Haplótipos. 


\section{Abstract}

Haplotype information has a central role in the understanding and diagnosis of certain illnesses, and also for evolution studies. Since that type of information is hard to obtain directly, computational methods to infer haplotype from genotype data have received great attention from the computational biology community. Unfortunately, haplotype inference is a very hard computational biology problem and the existing methods can only partially identify correct solutions. I present neural network models that use different properties of the data to predict when a method is more prone to make errors. I construct models for three different Haplotype Inference approaches and I show that our models are accurate and statistically relevant. The results of our experiments offer valuable insights on the performance of those methods, opening opportunity for a combination of strategies or improvement of individual approaches. I formally demonstrate that Linkage Disequilibrium (LD) and heterozygosity are very strong indicators of Switch Error tendency for four methods studied, and I delineate scenarios based on LD measures, that reveal a higher or smaller propension of the HI methods to present inference errors, so the correlation between LD and the occurrence of errors varies among regions along the genotypes. I present evidence that considering windows of length 10 , immediately to the left of a SNP (upstream region), and eliminating the non-informative SNPs through Fisher's Test leads to a more suitable correlation between LD and Inference Errors. I apply Multiple Linear Regression to explore the relevance of several biologically meaningful properties of the genotype sequences for the accuracy of the haplotype inference results, developing models for two databases (considering only Humans) and using two error metrics. The accuracy of our results and the stability of our proposed models are supported by statistical evidence.

Keywords: Linear Regression. Statistical Analysis. SNPs, Haplotypes. Genotype Data. Haplotype Inference. 


\section{List of Figures}

1.1 Single nucleotide polymorphisms(SNPs) $\ldots \ldots \ldots \ldots \ldots$

1.2 Meiosis Overview . . . . . . . . . . . . . . . . . . . . . . 19

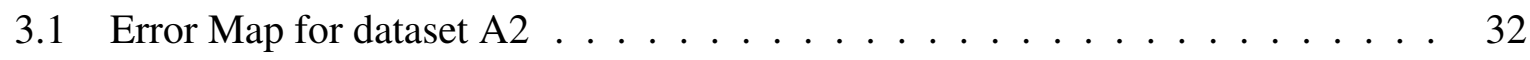

3.2 Relationship between LD and error abundance in dataset A2 . . . . . . . . 33

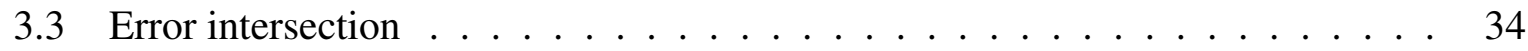

3.4 Venn diagram for the errors loci $\ldots \ldots \ldots$. . . . . . . . . . . . . . 37

3.5 Comparison of the Switch Error scores of the three best methods analyzed . . . 37

4.1 HybHap Tree . . . . . . . . . . . . . . . . . . . . . . . . 42

4.2 Example of Algorithm Execution . . . . . . . . . . . . . . . . . . . . 43

4.3 Computational Time (in seconds) and Error Rate attained in each dataset class . 47

5.1 Outline of the procedure to create the Meta-Data Base . . . . . . . . . . . 53

5.2 Correlation of predicting variable $H T Z$ with response variable Error Rate . . . 57

6.1 Boxplot for the average of ratios . . . . . . . . . . . . . . . 70

6.2 Evaluation of the MLR models proposed for the Error Rate and Switch Error . 78

6.3 Decision Tree Models . . . . . . . . . . . . . . . . . . . . . . . 80

7.1 Correlation between viral load and CD4+ cells count . . . . . . . . . . . 87

7.2 ROC curves considering results of 50 runs of three Models. . . . . . . . . . . 93

7.3 Venn Diagram of patient's consensus response predicted incorrectly . . . . . . 94

7.4 Decision Tree fitted considering CD4+ count at start of treatment. . . . . . . 95

7.5 Decision Tree fitted considering Viral Load at start of treatment. . . . . . . . . 95

7.6 Decision Trees estimated considering all codons of RT and PR . . . . . . . 95

7.7 Decision Trees estimated considering all codons of RT and PR for each model . 96 


\section{List of Tables}

3.1 Distinct Haplotype Fragments Found . . . . . . . . . . . . . . . . . . . 28

3.2 Comparison Results . . . . . . . . . . . . . . . . . . . 30

3.3 Switch Error Results. . . . . . . . . . . . . . . . . . . . . 31

3.4 Error Rate Measures for ten Datasets with 1000 random SNPs each . . . . . . . 35

3.5 Switch Error Measures for ten Datasets with 1000 random SNPs each . . . . . 35

4.1 Comparison Results . . . . . . . . . . . . . . . . . . . . 47

5.1 Correlation Found Using the Individuals Properties Base. . . . . . . . . . . 56

5.2 Correlation Found Using the SNPs Properties Base. . . . . . . . . . . . . . 56

5.3 Error Rate T-test's p-values of Nets with Single Input - Individuals Base . . . . 59

5.4 Switch Error T-test's p-values of Nets with Single Input - Individuals Base . . . 60

5.5 Comparison of the Best Single and Double-input Models - Individuals Base . . 60

5.6 Error Rate T-test's p-values of Nets with Single Input - SNPs Base . . . . . . . 61

5.7 Switch Distance T-test's p-values of Nets with Single Input - SNPs Base . . . . 62

5.8 Comparison of the Best Single and Double-input Models - SNPs Base . . . . . 62

6.1 Average ratios obtained by each LD mosaic for Switch Error . . . . . . . . . . 69

6.2 Correlation between HTZ, LD and Inference Error considering a specific LD

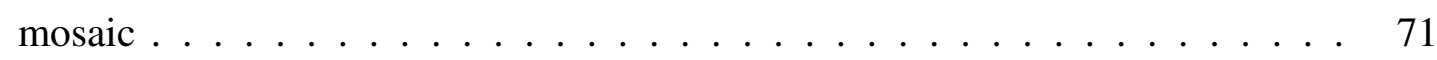

6.3 MLR models estimated for Individuals Base using the Step-Wise Method. . . . 73

6.4 MLR models estimated for the SNPs Base considering different LD levels . . . 74

6.5 Hypothesis test's p-values . . . . . . . . . . . . . . 76

6.6 Comparison of Relative Error ( $e$ ) obtained in MLR models, segmented in inter-

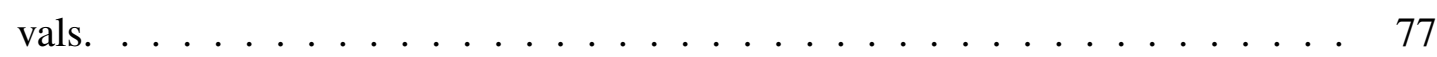

6.7 Accuracy of the decision trees constructed . . . . . . . . . . . . 80

7.1 Performance of Models for each class . . . . . . . . . . . . . . . . 92 
7.2 The average(standard deviation) of Viral Load and CD4+ for EASY and HARD

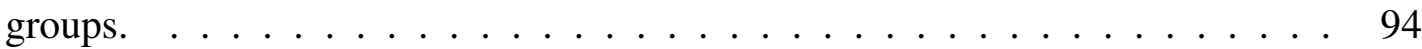

7.3 The distribution of the classes in EASY and HARD group. . . . . . . . . . 95

7.4 Relative Frequency of Patients by Class, CD4+ Count and Viral Load. . . . . . 99 


\section{List of Acronyms}

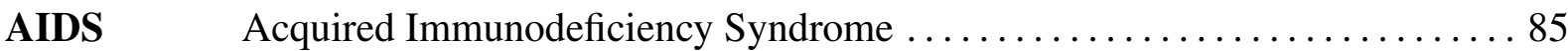

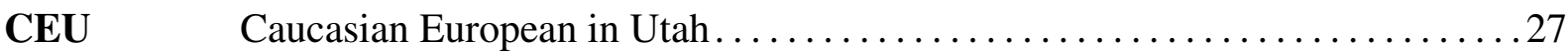

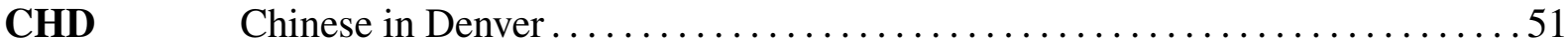

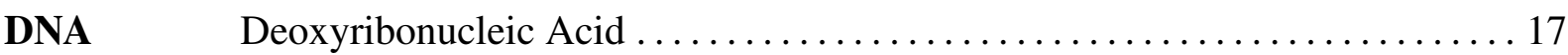

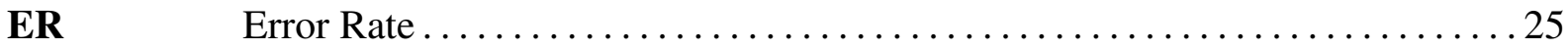

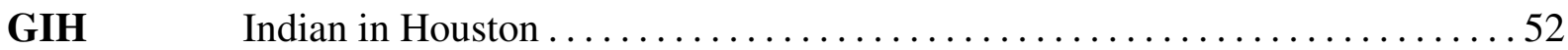

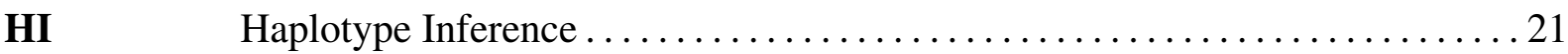

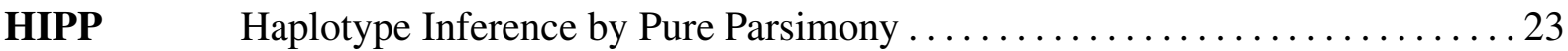

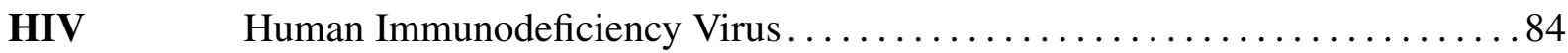

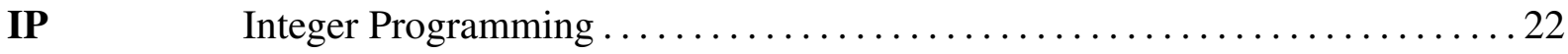

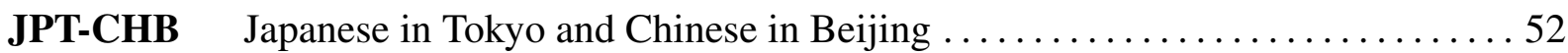

LD Linkage Disequilibrium $\ldots \ldots \ldots \ldots \ldots \ldots \ldots \ldots \ldots \ldots \ldots \ldots \ldots \ldots \ldots \ldots \ldots \ldots \ldots \ldots \ldots \ldots$

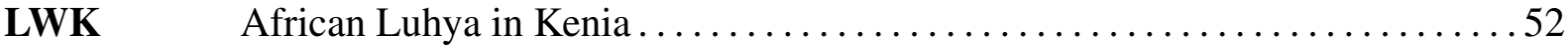

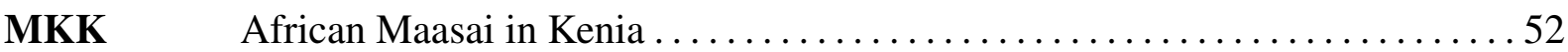

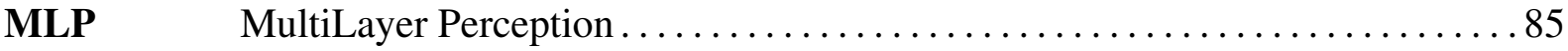

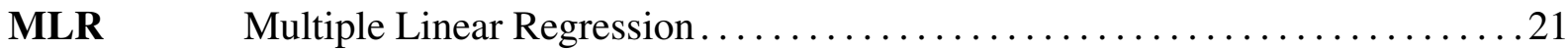

NIH National Institutes of Health................................ 20

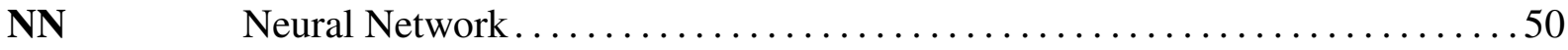

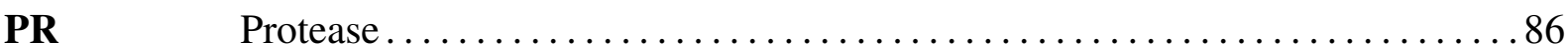

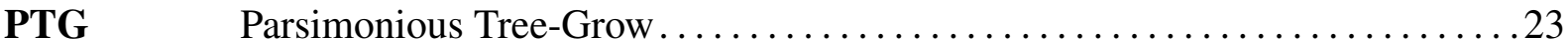

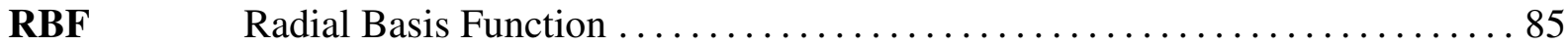

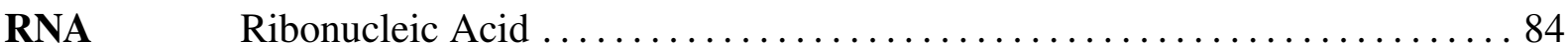


SMOTE $\quad$ Synthetic Minority Over-sampling Technique $\ldots \ldots \ldots \ldots \ldots \ldots \ldots \ldots \ldots$

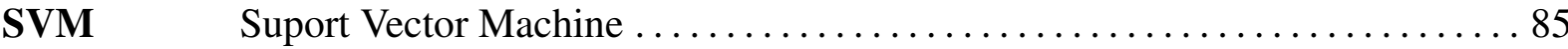

TSI

Italians in Toscana ................................... 52

VL

Viral Load. 86 


\section{Contents}

1 Introduction 17

1.1 Biological Background . . . . . . . . . . . . . . . . 18

1.2 Structure of the thesis . . . . . . . . . . . . . . . 21

2 Haplotype Inference Problem 22

2.1 Haplotype Inference Methods . . . . . . . . . . . . . . . . . . 22

2.2 Error measures for Haplotype Infence . . . . . . . . . . . . . . . . 25

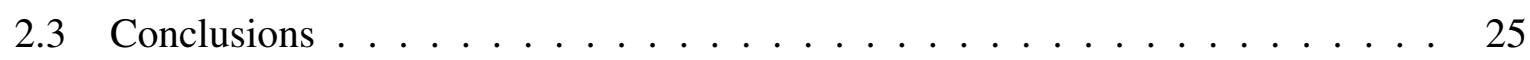

3 Evaluation of Haplotype Inference Methods 26

3.1 Experiments Design . . . . . . . . . . . . . . . . 26

3.2 Experiments Results . . . . . . . . . . . . . . . . . . . . 29

3.2.1 Comparison using Metrics . . . . . . . . . . . . . . . . . 29

3.2 .2 Considering Error Loci . . . . . . . . . . . . . . . . . . 30

3.3 Accuracy Assessment in General . . . . . . . . . . . . . . . . . 34

3.3.1 Initial Error Measure Analysis . . . . . . . . . . . . . . . . . 34

3.3.2 Error Locus Analysis for the Best Performing Methods . . . . . . . . . 36

3.4 Discussion and Conclusion $\ldots \ldots \ldots \ldots \ldots$

4 A Proposed Haplotype Inference Method 39

4.1 Computing The Markov Chain . . . . . . . . . . . . . . 40

4.2 The HybHap Method $\ldots \ldots \ldots$. . . . . . . . . . . . . . 41

4.3 Experiments Design . . . . . . . . . . . . . . . . . 44

4.4 Experiments Results . . . . . . . . . . . . . . . . . 47

4.5 Discussion and Conclusions . . . . . . . . . . . . . . . 48

5 Prediction of Error in Haplotype Inference Methods through Neural Networks 50 
5.1 Haplotype Inference Methods Considered . . . . . . . . . . . . . . . . 51

5.2 Experiments Design . . . . . . . . . . . . . . . . 51

5.2.1 Genotype Data Base . . . . . . . . . . . . . . 51

5.2.2 Genotype Meta-Data Bases . . . . . . . . . . . . . . . 52

5.2.3 Selecting and Validating the Neural Network Models . . . . . . . . . . 54

5.2.4 Hypothesis Tests on the Results . . . . . . . . . . . . . . 55

5.2.4.1 Single Input Networks . . . . . . . . . . . . . . . 55

5.2.4.2 Double Input Networks . . . . . . . . . . . . . 55

5.3 Results and Discussion . . . . . . . . . . . . . . . . 56

5.3.1 Correlations between Properties and Error Measures . . . . . . . . . 56

5.3.2 Assessment of the Neural Network Models . . . . . . . . . . . . 58

5.4 Conclusions ............................... 61

\section{Predicting Error in Haplotype Inference}

$\begin{array}{ll}\text { through MLR } & 64\end{array}$

6.1 Linkage Disequilibrium versus Heterozygosity . . . . . . . . . . . . 65

6.1 .1 Selecting the more informative LD window . . . . . . . . . 66

6.1.2 Correlation between inference error and HTZ/LD . . . . . . . . . . . . 70

6.2 Multiple Linear Regression Models . . . . . . . . . . . . . . . . 71

6.2.1 Data Bases and Their Properties . . . . . . . . . . . . . 71

6.2 .2 MLR Models . . . . . . . . . . . . . . . . . . 72

6.2.3 MLR Models with Selected Regressors . . . . . . . . . . . . . 73

6.2 .4 MLR Models Accuracy . . . . . . . . . . . . . . . . 75

6.3 Decision Trees to Identify Scenarios $\ldots \ldots \ldots \ldots$

6.3.1 Design of Decision Tree Models . . . . . . . . . . . . . 79

6.3.2 Decision Tree Models Results . . . . . . . . . . . . . . . . 79

6.4 Discussion and Conclusions $\ldots \ldots \ldots \ldots$. . . . . . . . . . . . 81

$\begin{array}{lll}7 & \text { A practical application } & 84\end{array}$

7.1 HIV and AIDS treatment $\ldots \ldots \ldots \ldots$ 
7.2 Experiments Design . . . . . . . . . . . . . . . . . . . 86

7.2.1 Data preparation ........................ 86

7.2.2 Classifiers considered . . . . . . . . . . . . . . . . 89

7.3 Results . . . . . . . . . . . . . . . . . . . . . 91

7.3.1 Statistical Performance of the Methods _ . . . . . . . . . . . . . 91

7.3.2 Qualitative analysis of results . . . . . . . . . . . . 92

7.4 Discussion and Conclusions $\ldots \ldots \ldots$. . . . . . . . . . . . . . 97

8 Conclusions $\quad 102$

$\begin{array}{ll}\text { References } & 104\end{array}$ 


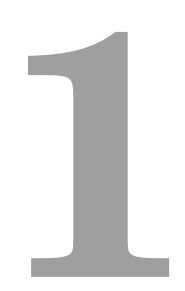

\section{Introduction}

And in the end, it's not the years in your life that count. It's the life in your years.

-ABRAHAM LINCOLN

An important challenge in biology is correlating differences in phenotypes with variations in the Deoxyribonucleic Acid (DNA). It is known that the human genome is highly similar for different individuals of the same population, and that some regions of the genomic DNA sequences are conserved along generations, with emphasis on coding regions. In these conserved regions, changes can occur in some specific alleles called Single Nucleotide Polymorphism (SNP). Besides a strong relation with phenotypes, SNPs can also be related with genetic diseases, and for that reason it is desirable to map them. Identifying these specific changes is not an easy task because it requires haplotype information. Diploid organisms have two copies of each chromosome, each copy of a given region is called an haplotype and a collection of these homologue chromosomes is called a genotype (Figure 1.1). Due to technological constraints, only the latter is available in large scale. Getting haplotype information directly is costly, therefore computational methods to infer haplotypes from genotype data at populational level are highly desirable. 


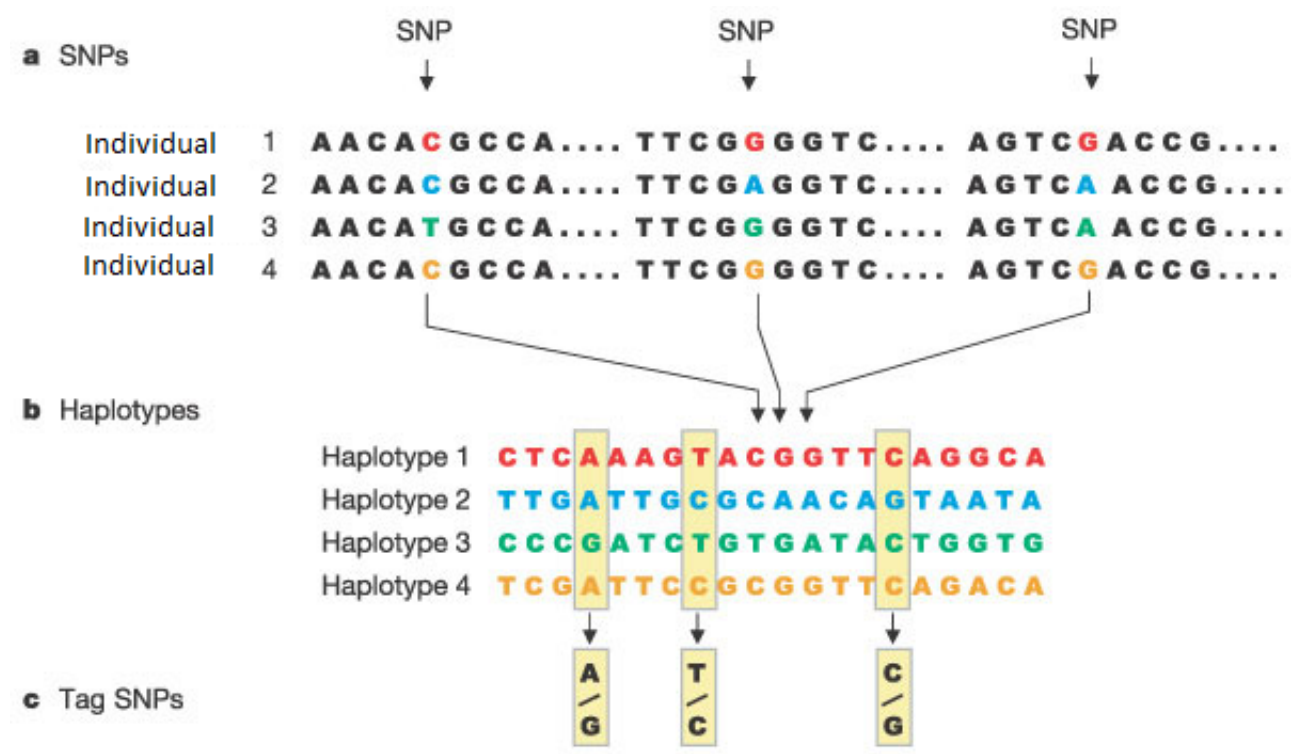

Figure 1.1: SNPs: (a) Single nucleotide polymorphisms(SNPs) are identified in DNA samples from multiple individuals. (b)Adjacent SNPs that are inherited together are compiled into haplotypes; (c) Tag SNPs within haplotypes are identified that uniquely identify those haplotypes. By genotyping the three tag SNPs shown in this figure, researchers can identify which of the four haplotypes shown here are present in each individual (Figure and description taken from The International HapMap Consortium (2003)).

Several computational methods have been proposed for inferring haplotypes from genotype data. Currently, the challenge is to infer haplotypes from large scale genotype, due to the high computational costs of these approaches and the high demand from actual applications. Though there are some comparative studies of haplotype inference methods, they are not actual and do not include more recent approaches or methods that are considered as good techniques for inferring haplotypes in large scale (ADKINS, 2004; XU et al., 2004).

\subsection{Biological Background}

Deoxyribonucleic acid (DNA), often found in nature in the form of a double-stranded chain, contains the specification of genetic instructions for the cellular development of all life forms and many viruses. Loci are locations (alleles) in the DNA sequence that can present one of four possible nucleotide bases: adenine (A), cytosine (C), guanine (G) and thymine (T). Among the nucleotides, $\mathrm{A}$ and $\mathrm{C}$ are from the family of components called Purines, while $\mathrm{G}$ and $\mathrm{T}$ are Pyrimidines. The DNA is usually packaged in one or more macromolecules called chromosomes. In this thesis, all data used are from humans, a diploid organism (meaning that 


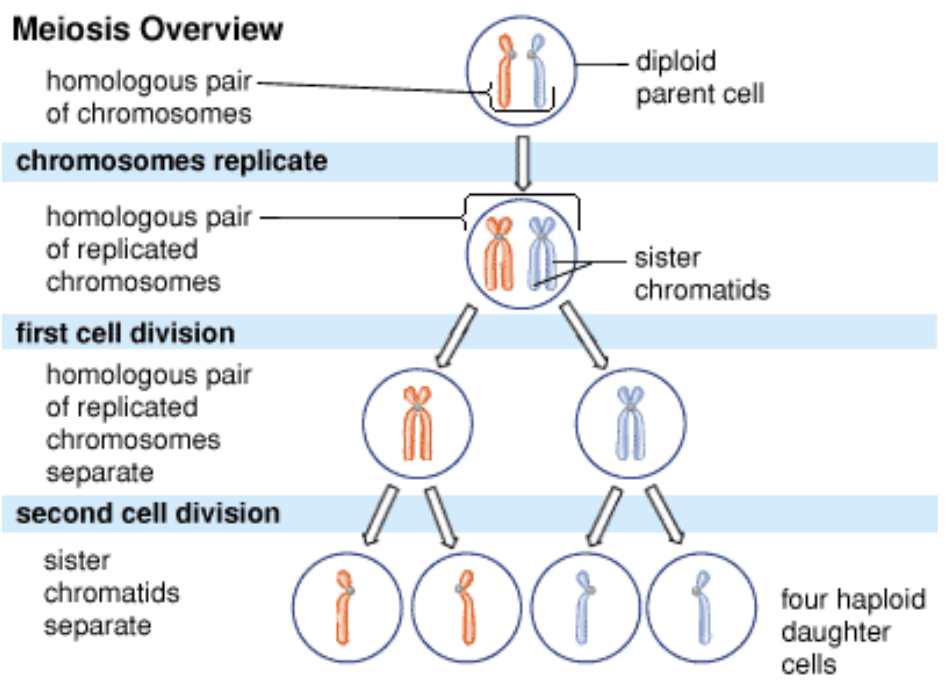

Figure 1.2: Meiosis Overview, Figure taken from Encyclopedia Britannica.

their chromosomes are organized in homologous pairs).

Two homologue chromosomes have very similar sequences. Loci that have the same nitrogenous bases in both molecules are called homozygous, while the others are called heterozygous (thus, for each trait or function there may be one or two alleles (one in each homologue chromosome). A healthy cell contains 23 pairs of chromosomes, each pair consisting of a chromosome inherited from the father and another from the individual's mother.

Mutations commonly occur in the genetic material, and generate variations in the population. Unfavorable (or deleterious) mutations can be reduced in the population through natural selection, while favorable mutations (beneficial or advantageous) can be accumulated, resulting in adaptive evolutionary changes. Mutations can occur in small (point mutations) or large scale (chromosomal mutations), and may be composed of the exchange of only one nucleotide for another, or even the elimination of a complete gene, or still a gene cluster.

The genetic inheritance that the individual receives is not an identical transmission of chromosomes through generations. In addition to mutations, the DNA sequences are target of recombination. In the process of formation of gametes, known as meiosis, chromosome pairs line up and exchange sequences between them, the result of this is a new cell with 23 new chromosomes, this process is called recombination. For example, if a diploid individual has in its cells a particular chromosome with alleles $(A, B, C, D)$ paired to $(a, b, c, d)$, the result of the recombination process may be $(\mathrm{a}, \mathrm{b}, \mathrm{C}, \mathrm{D})$ or $(\mathrm{a}, \mathrm{B}, \mathrm{C}, \mathrm{D})$, among other possibilities. 
Gametes that are generated by meiosis are called haploids (Figure 1.2), and they have half of the genetic material of diploid cells. Thus, an haplotype can be defined as a DNA sequence that is inherited from each parent of an individual. The union of gametes genetic material generates a novel and unique genotype. However, it is observed that many stretches of DNA remain unchanged through generations. These conserved regions are very often responsible for basic functions associated with the maintenance of life, and perpetuate the specific traits of the species. Abnormalities in these regions may be responsible for specific genetic diseases, such as in genes associated with the development of some types of cancers. Unfortunately, these mutations can also be transmitted by heritage. Changes in isolated alleles, whether or not related to the development of certain diseases, are considered Single Nucleotide Polymorphism (SNP) only if they occur in at least $1 \%$ of the population. The other cases are usually considered isolated mutations or sequencing errors.

Findings that genes can be inherited as groups from generation to generation had an impact in the scientific community, since the results of these genes made it possible to identify which ones are connected to the onset of certain diseases. The National Institutes of Health (NIH) initiated, in 2002, an international project, called HapMap (The International HapMap Consortium, 2003), which aims to compare the genomes of various individuals to identify exactly which regions of chromosomes are shared among them.

Unfortunately, mapping these shared areas is a major challenge because the methods applied for sequencing, in large-scale and low cost, fail for the identification of heterozygous (ambiguous) loci. The most commonly employed method for DNA amplification, PCR, as well as the techniques used for automatic sequencing, have limitations when it comes to regions with heterozygous loci. PCR synthesizes in little time a large amount of a DNA fragment. The main idea is to separate the two strands of DNA, using high temperatures, and to apply each of them as a template for synthesis of a complementary stand. This process is performed recursively, resulting in an exponential number of copies of the original DNA fragment. However, when this process is applied to regions heterozygous (in diploid organisms) amplification occurs in both sequences causing ambiguity in the identification of nucleotides (CLARK, 1990). That ambiguity could only be solved by having information about the original haplotypes. 
In this thesis we present insights on the problem of computational haplotype inference for large genotype datasets. Our observations are drawn from an extensive comparison of methods for haplotype inference using several datasets taken from HapMap (The International HapMap Consortium, 2003). We present neural network models that use different properties of the data to predict when a Haplotype Inference (HI) method is more prone to make errors. We construct models for three different $\mathrm{HI}$ approaches and we show that our models are statistically relevant. We formally demonstrate that Linkage Disequilibrium (LD) and heterozygosity are very strong indicators of Switch Error tendency for four methods studied. We also delineate scenarios based on LD measures, that reveal a higher or smaller propension of the HI methods to present inference errors, so the correlation between LD and the occurrence of errors varies among regions along the genotypes. We apply Multiple Linear Regression (MLR) to explore the relevance of several biologically meaningful properties of the genotype sequences for the accuracy of the haplotype inference results.

\subsection{Structure of the thesis}

This thesis is organized as follows. Chapter 2 gives a formal definition of the Haplotype Inference Problem, and presents related work, briefly introducing the best known Haplotype Inference methods. In Chapter 3, a preliminary evaluation of Haplotype Inference methods is done. In Chapter 4, a new method for Haplotype Inference is introduced. In Chapter 5, Neural Network models for estimating errors in Haplotype Inference methods are presented. In Chapter 6, an efficient approach based on Multiple Linear Regression for estimating errors is described. In Chapter 7, we used the framework analysis developed to explore insights on prediction of patients' response to anti-HIV therapies through machine learning. Finally, in Chapter 8, the concluding remarks and plans for future works are presented. 


\section{Haplotype Inference Problem}

A genotype (haplotype) can be computationally represented by a vector based on alphabet $\{0,1,2\}(\{0,1\})$, where a symbol 2 represents an ambiguous locus. Two haplotype vectors $h_{1}$ and $h_{2}$ are said to explain a genotype vector $g$, denoted $h_{1} \otimes h_{2}=g$, if each one of them has $n$ loci and, for each locus $i, 1 \leq i \leq n, h_{1}(i), h_{2}(i) \in\{0,1\}$ and follow the rule given by: (A) $h_{1}(i)=h_{2}(i)=g(i)$, if $g(i) \in\{0,1\}$; and (B) $h_{1}(i)=1-h_{2}(i)$, if $g(i)=2$.

The Haplotype Inference Problem basically consists of finding for each genotype $g$ two haplotypes $h_{1}$ and $h_{2}$ such that $h_{1} \otimes h_{2}=g$, i.e. $h_{1}$ and $h_{2}$ explain $g$ in a biologically plausible way. For instance, if $g=(0,1,2,2,1,2)$, possible solutions are $h_{1}=(0,1,0,0,1,0)$ and $h_{2}=(0,1,1,1,1,1)$, or still $h_{1}=(0,1,0,1,1,1)$ and $h_{2}=(0,1,1,0,1,0)$, among other possibilities. It is easy to see that there are $2^{k-1}$ candidate haplotype pairs to explain $g$, where $k$ is the number of ambiguous loci in $g$. Obviously, there are many solutions for each input $g$, so a biological model criterion is needed to define a good solution, such as the Pure Parsimony model.

\subsection{Haplotype Inference Methods}

Many combinatorial and statistical methods have been proposed for haplotype inference, such as the Clark Method (CLARK, 1990), Integer Programming (IP) formulations based on the parsimony principle (GUSFIELD, 2001, 2003; LANCIA; PINOTTI; RIZZI, 2004; HALLDóRSSON et al., 2004; BROWN; HARROWER, 2006), phylogeny based (GUSFIELD, 2002; DING; 
FILKOV; GUSFIELD, 2005), and Bayesian methods (STEPHENS; SMITH; DONNELLY, 2001; NIU et al., 2002). More recently, other methods were projected, such as methods based on Markov Chain Models (SUN; GREENWOOD; NEAL, 2007; WU; ZANG; CHAN, 2008) and haplotyping via Genetic algorithms (CHE; TANG; SONG, 2009).

There are two main biological models used to infer haplotypes: Pure parsimony and Perfect phylogeny. The parsimony-based methods search the solution space for the minimum distinct set of haplotypes that explain the genotype data. Unfortunately, the problem of inferring haplotypes by pure parsimony was considered NP-hard by (GUSFIELD, 2001). The perfect phylogeny-based methods build a tree structure and the haplotypes inferred should form a perfect phylogeny. Inferring haplotypes using this principle is a polynomial-time problem, but the assumption that the DNA sequences were not subject to recombination events is not realistic (DING; FILKOV; GUSFIELD, 2005).

Haplotype Inference by Pure Parsimony (HIPP) has been used by many approaches because of its innate simplicity and biological soundness. Initially, IP was applied to solve HIPP. There are three main problem formulations using IP. The first one, called RTIP (GUSFIELD, 2003), has an exponential number of constraints in the worst case, but it has been shown (BROWN; HARROWER, 2006) that when this formulation can resolve the input dataset, it may be faster than more recent IP formulations. Two independent groups (LANCIA; PINOTTI; RIZZI, 2004; HALLDóRSSON et al., 2004) have introduced polynomial-size IP formulations for HIPP, one of them known as PolyIP, however, this formulation is very slow for large datasets, according to (BROWN; HARROWER, 2006), who proposed a hybrid formulation with a polynomial number of constraints but faster than PolyIP, called HybridIP.

Other methods for HIPP were published. Among them, Parsimonious Tree-Grow (PTG) offers a good compromise of high accuracy at a relatively low computational complexity. The PTG method explains a set of $m$ genotypes of length $n$ in time $O\left(m^{2} n\right)$. The accuracy of PTG is demonstrated theoretically and also experimentally (LI et al., 2005), using comparisons involving HAPAR, HAPLOTYPER, HAPINFERX and PHASE. However, many operations in PTG are random, so it is necessary to run the method many times, and select the best solution using some metric, in order to have reliable results. The metric applied for quality is the number of distinct 
haplotypes inferred.

Methods based on Markov Chain Model have been proposed successfully. The Markov Chain was applied to the HI problem originally by Eronen and colleagues (ERONEN; GEERTS; TOIVONEN, 2004). Later works improved this approach (SUN; GREENWOOD; NEAL, 2007; WU; ZANG; CHAN, 2008; ZHANG et al., 2008; ERONEN; GEERTS; TOIVONEN, 2006). These methods basically build a Markov chain where each state is a possible symbol (0 or 1$)$ and the transition probabilities are calculated from the input data. It is possible to estimate the probability that each candidate haplotype fragment will be part of the optimal solution. The number of haplotype candidates and probability combinations (as the original problem) is clearly exponential. For this reason, heuristics based on Dynamic Programming and Expectation Maximization algorithms have been proposed (SUN; GREENWOOD; NEAL, 2007; ZHANG et al., 2008). These heuristics search the solution space for a good explanation of the input data. The goal is to maximize the probability that a candidate haplotype will be part of resolution of several genotypes. Thus a haplotype vector is able to resolve a bigger number of genotypes and would be more plausible biologically. Based on this approach the software called Haplorec (ERONEN; GEERTS; TOIVONEN, 2006), available on the web, was developed; version Haplorec 2.3 is an improved version that solves very large datasets.

Although not as recent as Haplorec, PHASE (STEPHENS; SMITH; DONNELLY, 2001; STEPHENS; DONNELLY, 2003) and fastPHASE (SCHEET; STEPHENS, 2006) are considered good classical approaches for the HI Problem. These methods use maximum likelihood to estimate haplotype frequencies. The objective is determining the maximum value of this likelihood function. Such methods are stochastic and each execution of the program may result in a different solution, since the derivations are dependent on the initial configuration which is randomly selected. Basically, fastPHASE is a variation of PHASE for resolving large data sets.

Due to their computational performance, PTG, Haplorec and fastPHASE are good candidates for resolving genotypes in large scale. Some works in the literature compare haplotype inference methods (ADKINS, 2004; XU et al., 2004), but Haplorec and PTG are not included in those analyses, because they were released later. The techniques have different behavior and accuracy on distinct datasets. We can assume that the quality of the solution is closely related 
to the configuration and properties of the input genotypes. Our goal in next Chapter is to look closer into that connection.

\subsection{Error measures for Haplotype Infence}

The error metrics more frequently used for the HI Problem are Error Rate (ER) (NIU et al., 2002) and Switch Error (SE) (LIN et al., 2002). For the Error Rate, the correct haplotypes are aligned to the inferred ones, the number of mismatches is computed, and the Error Rate is given by the ratio between the number of mismatches and the total number of loci in the dataset. The Swith Error is calculated as $(N-1-S D) /(N-1)$, where $N$ denotes the number of heterozigous loci, and Switch Distance (SD) denotes the minimum number of block exchanges required between the two inferred haplotypes, in order to make them identical to the original ones (for more details see TINININI et al. (2010)).

\subsection{Conclusions}

Computational and statistical approaches are required for HI problem. Usually, more expansive algorithms have the best solutions (such as PHASE). The high computational costs of these methods is a problem for resolving large datasets. Therefore, new strategies for improving the results of the most efficient methods (algorithms with low computational costs) are required. 


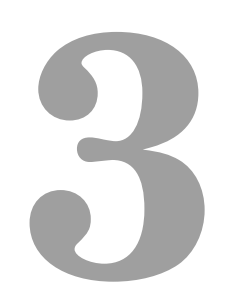

\section{Evaluation of Haplotype Inference Methods}

In this Chapter we developed an extensive analysis involving methods fastPHASE (SCHEET; STEPHENS, 2006), Haplorec (ERONEN; GEERTS; TOIVONEN, 2006), and PTG (LI et al., 2005), each of them is based on a different computational technique. These approaches were selected because they are deemed to be among the best algorithms in the literature, known to resolve very large datasets. For this benchmark, datasets collected from HapMap project were used. We evaluated the capacity of each method to rebuild the correct haplotype data set, and also the time required for finding a good solution. We also analyzed what type of characteristics of the dataset, such as conservation level, for instance, made a method more effective.

The rest of the Chapter is organized as follows. In Section 3.1, the design of the experiments and the data used are described. In Section 3.2, the results of our comparative analysis are shown. A discussion and concluding remarks are presented in Section 3.4. The results of this Chapter were presented in ROSA; GUIMARÃES (2010).

\subsection{Experiments Design}

The HapMap project (The International HapMap Consortium, 2003) is an important source of information about haplotypes shared among individuals from the same population. This international project was started by NIH (National Institute of Health) in 2002, with the goal of mapping these shared sequences. For experiments in this work, haplotypes were collected 
from HapMap Phase III, of chromosome 20 from a population of Trios Utah residents with Northern and Western European ancestry; this is 1 of 11 populations in HapMap Phase III, called Caucasian European in Utah (CEU). We selected dataset CEU because of the large number of individuals phased in it (88 individuals).

Chromosome 20 spans about 62 million DNA base pairs building blocks and represents approximately $2 \%$ of the total DNA in humans cells. This chromosome has between 700 and 800 genes. Many genetic diseases can be related with it. Changes in chromosome 20 have been identified in several types of cancer such as leukemia and lymphoma. Deletions or duplications of genetic material from chromosome 20 can have a variety of effects, including intellectual disability, delayed development, dysmorphic characters, skeletal abnormalities, and heart defects. The raw data presents 36258 SNPs and 88 individuals (two sequences for each individual). We mapped all haplotype fragments of length $100,200,400,800$, and 1600 to datasets in classes A, B, C, D, and E, respectively. For each class, the fragments with the smallest, the average, and the biggest number of distinct original haplotypes were selected to form three distinct datasets, called X1, X2, and X3, respectively, in which X can be A, B, C, or D. Since all the sequences in class E had virtually the same number of distinct haplotypes, this class has only one dataset, called E1. All together, 13 datasets were generated.

Each measure presented many candidate sets, for instance, there were 18 sets with 200 SNPs, 88 individuals and 13 distinct haplotypes (13 is the smallest number of distinct haplotypes found for a matrix 200x88 in CEU), for this reason, a random choice for selecting a representative set was needed. In the case of the set with 1600 SNPs, the mapping was discarded because the smallest number of distinct haplotypes found was 175 while the largest was 176 (really similar). Due to that, a subset was randomly selected in CEU with 1600 SNPs.

The purpose of mapping the occurrences of distinct haplotype fragments is to identify the abundance of easy and difficult datasets for resolution in a specific population. Table 3.1 shows the amount of distinct haplotypes found in CEU with the specific number of SNPs, and their respective number of representatives in CEU. With the integral data of the map, it is possible to identify the regions of chromosome 20 in CEU in which there is a concentration of haplotype fragments with similar properties, such as conserved sequences. According to the map, an 
increase in the SNPs number implies an increase in the number of distinct haplotype fragments. This is crucial for the analysis of the methods because different methods have distinct behaviors when resolving genotypes originated from more conserved or from more recombined sequences.

Table 3.1: Distinct Haplotype Fragments Found: Number of Distinct Haplotypes (DH) in Caucasian European in Uta (CEU), and their respective number of representatives.

\begin{tabular}{cccc}
\hline $\mathbf{N}^{\mathbf{o}}$ SNPs & Least DH & Larger DH & Average DH \\
\hline 100 & $13 / 7$ & $175 / 16$ & $101 / 370$ \\
200 & $38 / 18$ & $175 / 671$ & $145 / 330$ \\
400 & $102 / 2$ & $176 / 3187$ & $168 / 919$ \\
800 & $151 / 57$ & $176 / 15923$ & $175 / 12002$ \\
\hline
\end{tabular}

The metrics used in the benchmark were Error Rate (NIU et al., 2002), Switch Error (LIN et al., 2002), computational time, and number of distinct haplotypes inferred. The error metrics are based on known haplotype sets, and tell us about the capacity that a method has to correctly infer a haplotype set from a genotype set. The computational time is an empiric metric used for estimating computational cost. Although in general it is not the best technique for that, in this case, theoretical analysis cannot be applied to all methods. The number of distinct haplotypes is an important metric because the parsimony principle is being considered, and methods that find a minimal solution set are required. Time and number of distinct haplotypes are quantitative metrics.

Error Rate considers the rate of haplotype loci inferred incorrectly in all haplotype loci previously known. Switch Error is the proportion between the number of genotype ambiguous loci and the number of fragments changes needed between two specific haplotype vectors used to explain a specific genotype. These are considered quality metrics. Basically, Error Rate measures locus to locus individually, while Switch Error considers the neighbor loci. The goal here is to minimize the Error Rate and maximize the Switch Error (maximum value is 1).

For the comparison experiments, PTG was implemented in MATLAB 2008, for method Haplorec (haplotype inference based on Markov chains) the software version 2.3 was used, and for fastPHASE the version 1.2.3 for windows was used. The experiments run individually in a computer with an Intel Quad Core $2.33 \mathrm{GHz}$ processor, with $3 \mathrm{~GB}$ of RAM. Although there is a version of PTG available on the author's webpage, we have chosen to develop our own version of 
the method, because, since PTG is based on random operations, in order to find reliable results, PTG needs to be executed many times (in our case, we chose 30 runs), taking the best output. In our experiments, the smaller number of distinct haplotypes was used as metric for selecting the best solution.

\subsection{Experiments Results}

The measures used in the comparison were Error Rate, number of distinct haplotypes inferred, and computational time used to solve each instance. Algorithms Haplorec and fastPHASE were taken from their respective loci in the web.

\subsubsection{Comparison using Metrics}

Considering the qualitative measures, Haplorec and fastPHASE had similar performances. In datasets A and B, fastPHASE had lower Error Rate, this demonstrates the capacity of this method to deal well with conserved sequences. Haplorec was the best in datasets C and D (except for D2), but failed to resolve dataset E1. While fastPHASE took 54 minutes to find a solution with Error Rate 9,25\%, Haplorec needed only 2 minutes to find a solution with Error Rate 9,23\%. These two methods had similar Error Rate in all tests; the difference between them was not superior to $2 \%$ in each instance, however fastPHASE was more expensive computationally: while Haplorec took only seconds or a few minutes to resolve an instance, fastPHASE would take several minutes or hours to do the same task. Nonetheless, Haplorec could not handle the more difficult dataset, E1.

The results are shown in Tables 3.2 and 3.3. For each experiment it is given the execution time, the number of distinct haplotypes inferred, the error rate and switch error attained. Method PHASE was not included in the experiments because it failed to resolve even the smallest dataset (A1) in reasonable time. The Haplorec method failed to resolve dataset E1, returning several errors for this instance of the problem.

The experiments show the superior accuracy of fastPHASE and Haplorec considering Error Rate, but they both require a considerable computational time. Although the PTG method 
Table 3.2: Comparison Results: Error Rate (ER), Distinct Haplotypes found (DH), Time in seconds (s), minutes $(\mathrm{m})$ or hours (h).

\begin{tabular}{l|ccc|ccc|ccc}
\hline Set & \multicolumn{3}{c}{ PTG } & \multicolumn{3}{c}{ Haplorec } & \multicolumn{3}{c}{ fastPHASE } \\
& ER & DH & Time & ER & DH & Time & ER & DH & Time \\
\hline A1 & $0,35 \%$ & 12 & $21,24 \mathrm{~s}$ & $0,01 \%$ & 12 & $3 \mathrm{~s}$ & $0,00 \%$ & 13 & $13 \mathrm{~m}$ \\
A2 & $61 \%$ & 118 & $40,48 \mathrm{~s}$ & $3,26 \%$ & 98 & $5 \mathrm{~s}$ & $1,96 \%$ & 96 & $11 \mathrm{~m}$ \\
A3 & $12,98 \%$ & 166 & $53,88 \mathrm{~s}$ & $8,97 \%$ & 176 & $9 \mathrm{~s}$ & $8,97 \%$ & 176 & $5 \mathrm{~m}$ \\
B1 & $3,17 \%$ & 46 & $1,16 \mathrm{~m}$ & $0,70 \%$ & 64 & $10 \mathrm{~s}$ & $0,10 \%$ & 44 & $10 \mathrm{~m}$ \\
B2 & $9,54 \%$ & 155 & $1,81 \mathrm{~m}$ & $4,65 \%$ & 161 & $19 \mathrm{~s}$ & $4,21 \%$ & 158 & $25 \mathrm{~m}$ \\
B3 & $10,01 \%$ & 168 & $1,62 \mathrm{~m}$ & $6,05 \%$ & 176 & $20 \mathrm{~s}$ & $5,78 \%$ & 176 & $10 \mathrm{~m}$ \\
C1 & $3,65 \%$ & 112 & $2,63 \mathrm{~m}$ & $0,75 \%$ & 120 & $21 \mathrm{~s}$ & $1,09 \%$ & 115 & $54 \mathrm{~m}$ \\
C2 & $13,87 \%$ & 169 & $3,75 \mathrm{~m}$ & $6,63 \%$ & 174 & $49 \mathrm{~s}$ & $7,65 \%$ & 176 & $52 \mathrm{~m}$ \\
C3 & $12,53 \%$ & 174 & $3,73 \mathrm{~m}$ & $8,78 \%$ & 176 & $49 \mathrm{~s}$ & $9,49 \%$ & 176 & $25 \mathrm{~m}$ \\
D1 & $13,27 \%$ & 176 & $7,89 \mathrm{~m}$ & $10,48 \%$ & 176 & $3,5 \mathrm{~m}$ & $10,61 \%$ & 176 & $41 \mathrm{~m}$ \\
D2 & $11,47 \%$ & 173 & $7,34 \mathrm{~m}$ & $7,26 \%$ & 176 & $1,5 \mathrm{~m}$ & $7,07 \%$ & 176 & $1 \mathrm{~h} 40 \mathrm{~m}$ \\
D3 & $12,34 \%$ & 175 & $7,67 \mathrm{~m}$ & $9,23 \%$ & 176 & $2 \mathrm{~m}$ & $9,25 \%$ & 176 & $54 \mathrm{~m}$ \\
E1 & $14,19 \%$ & 174 & $16,98 \mathrm{~m}$ & - & - & - & $11,34 \%$ & 176 & $3 \mathrm{~h}$ \\
\hline
\end{tabular}

had worst accuracy in all tests, it was never by a large difference. On the other hand, the computational costs were much lower than the more accurate method fastPHASE. It is important to highlight that, for the hardest dataset, E1, while fastPHASE took 3 hours and Haplorec failed to deliver a result, PTG finished in 17 minutes with a result only 3 percentage points worse than that of fastPHASE.

The results considering Switch Error (Table 3.3) show that Haplorec and fastPHASE have a similar performance in this measure too. The highest difference among these methods was 0,006 (dataset A2), while the performance of PTG was really poor considering this metric. The standard deviation of Error Rate was also very close for Haplorec and fastPHASE, 0,023 and 0,024, respectively. Although fastPHASE and Haplorec had close performances, these numbers do not tell if the errors in the haplotypes inferred occur in the same sequence positions or not; we investigated further on the aspect of superposition of the loci wrongly resolved.

\subsubsection{Considering Error Loci}

The localization of errors in each method is very important for suggesting the type of sequences that are prone to to errors in a given method. So we mapped by individual and by SNP the positions where each method went wrong, as considered in Error Rate. We built an error map for the results of each dataset with each method. We verified that all PTG error maps 
follow a very scattered pattern over all datasets, which is expected, since it does not consider any type of neighboring or statistical information. On the other hand, fastPHASE and Haplorec both consider statistical factors (although different ones), based on biological principals and they seem to be sensitive to surrounding signals. We plotted the error maps superposed for each dataset for these two methods, and we observed that the different statistics used lead to errors mostly in different positions.

Table 3.3: Switch Error Results.

\begin{tabular}{cccc}
\hline Set & PTG & Haplorec & fastPHASE \\
\hline A1 & 0,994 & 0,999 & 1 \\
A2 & 0,856 & 0,980 & 0,986 \\
A3 & 0,623 & 0,918 & 0,917 \\
B1 & 0,938 & 0,991 & 0,996 \\
B2 & 0,714 & 0,980 & 0,982 \\
B3 & 0,770 & 0,956 & 0,960 \\
C1 & 0,856 & 0,983 & 0,987 \\
C2 & 0,575 & 0,973 & 0,979 \\
C3 & 0,623 & 0,952 & 0,953 \\
D1 & 0,521 & 0,937 & 0,938 \\
D2 & 0,599 & 0,977 & 0,982 \\
D3 & 0,558 & 0,958 & 0,961 \\
E1 & 0,535 & - & 0,959 \\
\hline
\end{tabular}

In Figure 3.1 we present the superposed error maps of fastPHASE and Haplorec for Dataset A2. It is possible to define two main areas of different error concentration. The bigger area, which we will call Area 1, contains the first 70 SNPs, and the smaller area, which we will call Area 2, contains the final 30 SNPs. For both methods, Area 1 contains a very low population of error, whereas Area 2 presents errors much more frequently.

We used Linkage Disequilibrium (LD) to compute how conserved each one of these two regions is. When the sequence is highly conserved, the value of measure LD tends to 1 (maximum value), while it tends to 0 when the individuals' samples do not share sequence among them. Still considering dataset A2, Area 1 presents LD = 0,2649 and Area 2 has LD = 0,1352. In general, for the more conserved datasets, in the more conserved regions fastPHASE and Haplorec both had superior performance. That same type of behavior was observed in the error maps of all the other datasets.

There is an inverse relationship between Error Rate and LD, which is depicted in Figure 
3.2. In SNPs with low LD (least conserved), fastPHASE and Haplorec did more inference mistakes. Regions with high LD are prone to the occurrence of homozygous loci, so a hypothetical explanation for this behavior would be the absence of heterozygous loci, however, Areas 1 and 2 had the same abundance of ambiguous loci: $30 \%$ of total loci in each area. A careful study of the curves in Figure 3.2 leads to the conclusion that LD is not the only factor involved in the Error Rate, although it is certainly a relevant one.

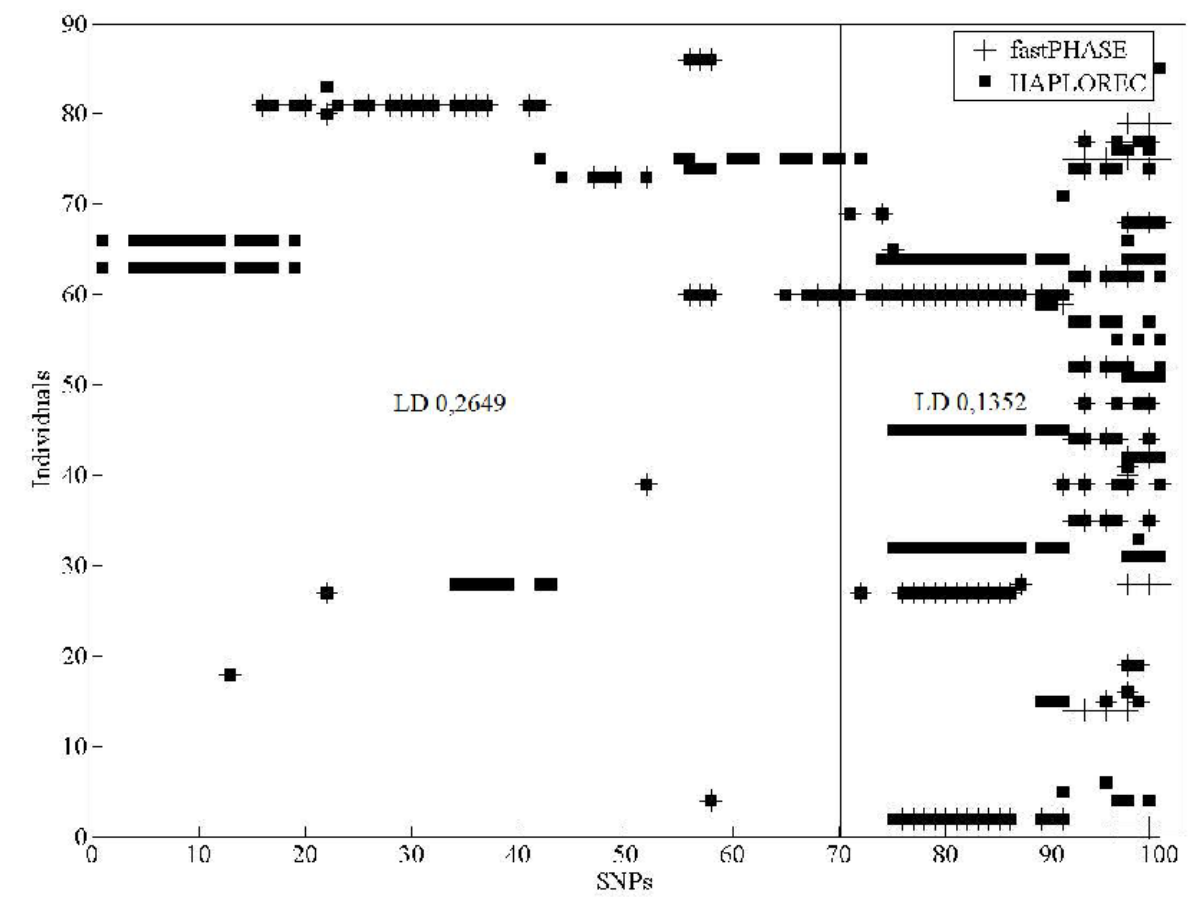

Figure 3.1: Error Map for dataset A2: axis $X$ presents 100 SNPs and in axis $Y$ the 88 individual of this sample.

Differently, for the PTG method we could not identify specific regions where the method made more errors; the errors occurred in a more scattered fashion along the sequences. However, we observed that genotype fragments that have original haplotype patterns as a sequence of one repeated symbol (such as $h_{1}=0^{k}$ and $h_{2}=1^{k}$, for some value $k$, for instance), are responsible for most errors observed in PTG. Basically, the errors in method PTG can be explained by its random behavior, and in most cases, there is no relationship with data distribution (genotypes).

The sequence positions where all methods failed to solve are somewhat difficult to explain, because they represent exceptions to the biological principles used to guide the methods. 


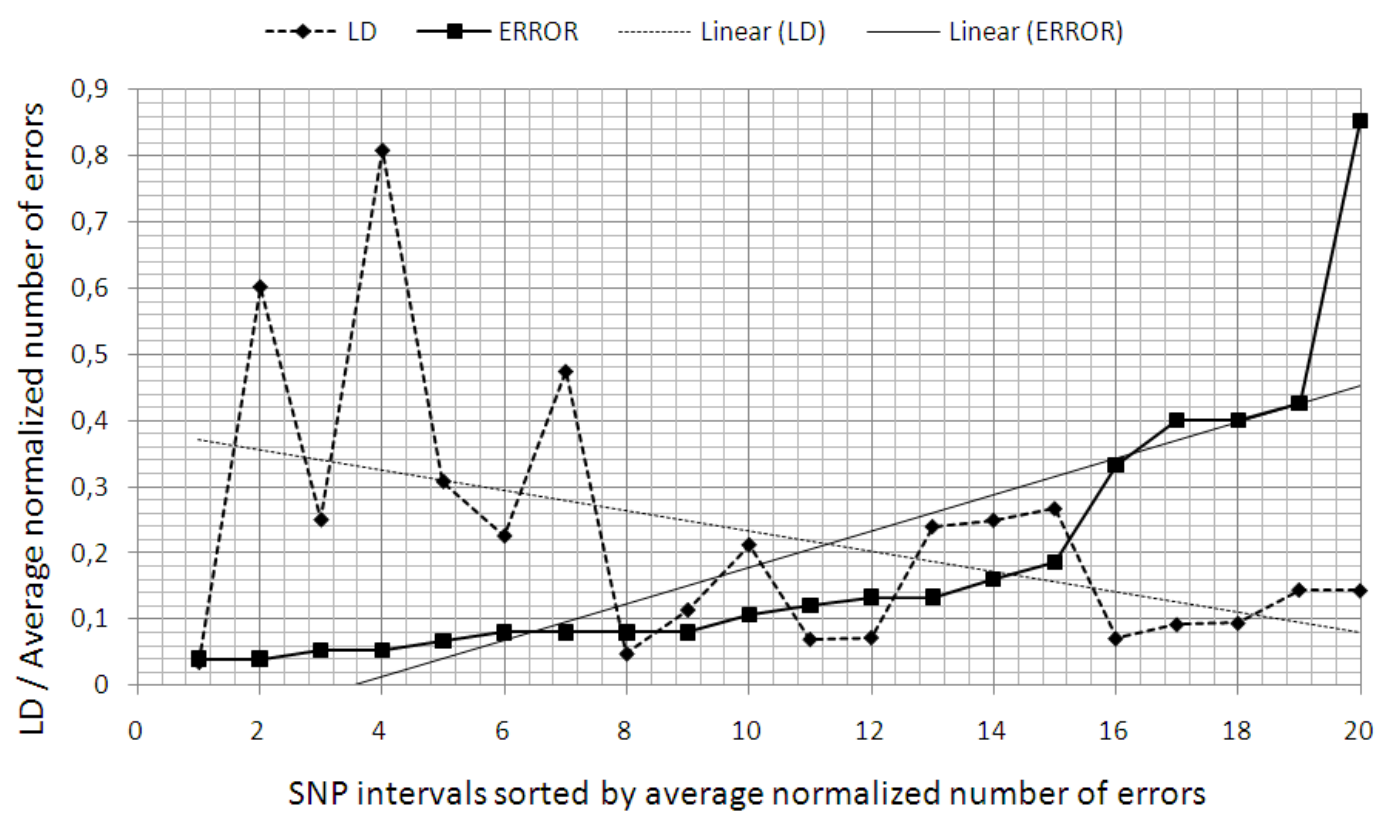

Figure 3.2: Relationship between $L D$ and error abundance in dataset $A 2$ : In axis $X$ the SNPs are grouped in subsets of 5 neighbors; in axis $\mathrm{Y}$ the averages of LD and \#errors, where the error abundance was normalized to the interval $[0,1]$ and then averaged.

No method in the literature seems to be able to foresee or deal with those cases based only on genotype data. Fortunately, we observed that those loci are relatively few. In our experiments, most of the loci have been correctly explained by at least one of the three methods. In Figure 3.3, we show four diagrams, each one with the average number of genotype loci inferred incorrectly in datasets A, B, C and D. As can be seen in Figure 3.3, the average number of errors common to all three methods when dealing with dataset $\mathrm{A}$ is given by (fastPHASE(A) $\cap$ Haplorec(A) $\cap$ PTG $(A))=77$, when the total number of loci in A was 962, that is, only $8 \%$. In a similar way, the ratio of errors common to all three methods in datasets $\mathrm{B}, \mathrm{C}$, and $\mathrm{D}$, were $5 \%, 6 \%$, and $8 \%$, respectively.

Another interesting observation is that, from the point of view of the algorithms, as the length of the sequences grows, the number of errors of each algorithm also grows, but not at the same rate. 
(A)

(B)
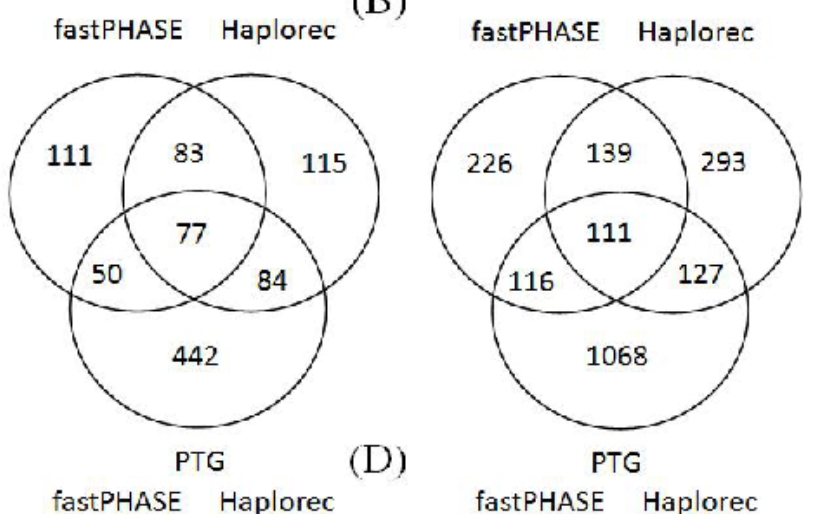

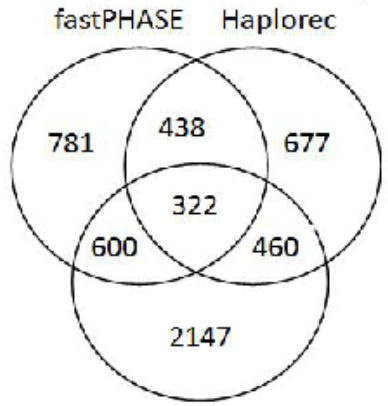

PTG

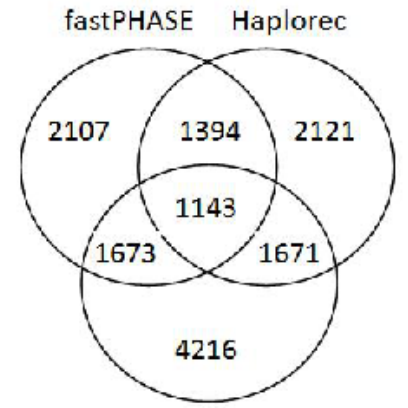

PTG

Figure 3.3: Error intersection: error average quantity in loci identified in the of datasets $\mathrm{A}, \mathrm{B}, \mathrm{C}$, and D.

\subsection{Accuracy Assessment in General}

\subsubsection{Initial Error Measure Analysis}

We considered the Error Rate and Switch Error measures of six of the best known haplotype inference methods, Beagle (BROWNING; BROWNING, 2009), CollHaps (TINININI et al., 2010), Haplorec, PTG, fastPHASE, and HMC (ZHANG et al., 2008). For that, we used ten datasets also taken from HapMap (The International HapMap Consortium, 2003), with 1000 random SNPs each. Tables 3.4 and 3.5 present the average Error Rate and the Switch Error, respectively, obtained in general and by specific datasets. Interestingly, we note that all six methods had better performance with set \#2, considering both metrics. On the other hand, the worst performances of the methods were scored with three different datasets $(6,9$ and 10) for Error Rate, and with two other datasets ( 3 and 8 ) for Switch Error; hence we assume that there is no dataset that is clearly the most difficult.

Considering both the Error Rate and the Switch Error metrics, the order of average 
Table 3.4: Error Rate Measures for ten Datasets with 1000 random SNPs each. Values in bold indicate the worst performance of each method.

\begin{tabular}{ccccccc}
\hline Set & Beagle & CollHaps & Haplorec & PTG & fastPHASE & HMC \\
\hline 1 & $7.53 \%$ & $9.59 \%$ & $8.24 \%$ & $11.54 \%$ & $7.48 \%$ & $8.48 \%$ \\
2 & $5.74 \%$ & $8.20 \%$ & $6.63 \%$ & $10.21 \%$ & $5.25 \%$ & $6.68 \%$ \\
3 & $8.08 \%$ & $9.09 \%$ & $8.22 \%$ & $11.10 \%$ & $8.02 \%$ & $8.05 \%$ \\
4 & $8.53 \%$ & $10.25 \%$ & $8.75 \%$ & $12.25 \%$ & $8.26 \%$ & $8.88 \%$ \\
5 & $8.18 \%$ & $10.47 \%$ & $8.35 \%$ & $12.89 \%$ & $8.03 \%$ & $8.20 \%$ \\
6 & $10.70 \%$ & $\mathbf{1 2 . 1 8 \%}$ & $\mathbf{1 0 . 2 3 \%}$ & $13.58 \%$ & $9.65 \%$ & $10.74 \%$ \\
7 & $10.22 \%$ & $11.84 \%$ & $9.87 \%$ & $13.77 \%$ & $9.43 \%$ & $10.11 \%$ \\
8 & $9.72 \%$ & $11.39 \%$ & $9.29 \%$ & $13.62 \%$ & $8.53 \%$ & $10.41 \%$ \\
9 & $\mathbf{1 0 . 8 7 \%}$ & $10.11 \%$ & $9.67 \%$ & $\mathbf{1 4 . 0 7 \%}$ & $8.96 \%$ & $\mathbf{1 1 . 3 1 \%}$ \\
10 & $9.80 \%$ & $11.49 \%$ & $9.58 \%$ & $13.45 \%$ & $\mathbf{9 . 7 7 \%}$ & $10.41 \%$ \\
\hline Avg. & $8.94 \%$ & $10.46 \%$ & $8.88 \%$ & $12.65 \%$ & $8.34 \%$ & $9.33 \%$ \\
\hline
\end{tabular}

Table 3.5: Switch Error Measures for ten Datasets with 1000 random SNPs each. Values in bold indicate the worst performance of each method.

\begin{tabular}{ccccccc}
\hline Set & Beagle & CollHaps & Haplorec & PTG & fastPHASE & HMC \\
\hline 1 & 0.9752 & 0.7929 & 0.9717 & 0.5314 & 0.9801 & 0.9719 \\
2 & 0.9808 & 0.8695 & 0.9798 & 0.5805 & 0.9854 & 0.9786 \\
3 & $\mathbf{0 . 9 4 4 5}$ & 0.8386 & $\mathbf{0 . 9 5 1 5}$ & 0.5110 & $\mathbf{0 . 9 5 8 2}$ & $\mathbf{0 . 9 4 4 1}$ \\
4 & 0.9580 & 0.7581 & 0.9600 & 0.5068 & 0.9674 & 0.9501 \\
5 & 0.9699 & 0.7764 & 0.9727 & 0.5167 & 0.9772 & 0.9709 \\
6 & 0.9545 & 0.7281 & 0.9611 & 0.5139 & 0.9690 & 0.9477 \\
7 & 0.9710 & 0.7439 & 0.9743 & 0.5232 & 0.9777 & 0.9713 \\
8 & 0.9535 & $\mathbf{0 . 7 0 3 3}$ & 0.9599 & $\mathbf{0 . 4 9 9 9}$ & 0.9694 & 0.9390 \\
9 & 0.9531 & 0.7381 & 0.9588 & 0.5129 & 0.9700 & 0.9444 \\
10 & 0.9648 & 0.7627 & 0.9672 & 0.5228 & 0.9719 & 0.9622 \\
\hline Avg. & 0.9625 & 0.7711 & 0.9657 & 0.5219 & 0.9726 & 0.9580 \\
\hline
\end{tabular}

accuracy measures attained by the six methods is the same, with fastPHASE being the best method, followed by Haplorec and Beagle; these three have Error Rate between $8 \%$ and 9\%, and Switch Error between 0.96 and 0.98 (recall that the Switch Error values can be between 0 and 1, where 1 represents a perfect score). Since the PTG method presented the worst averages, with virtually random Switch Error scores, it is excluded from the more detailed analysis in this section. We include PTG later in the regression analysis, to try to build some insight on possible causes for its poor performance. Methods CollHaps and HMC could not deal with experiments with larger datasets, so they are excluded from all reports that follow. Figure 3.5 shows the results of Switch Error of the three best HI methods, considering datasets with up to 10,000 SNPs. 


\subsubsection{Error Locus Analysis for the Best Performing Methods}

The specific positions where the errors occurred in the ten datasets of 1000 SNPs used in the experiments above were annotated for the three best performing methods, fastPHASE, Haplorec, and Beagle. Those loci were then compared among themselves, in order to analyze how many of them would be common between two or even all three methods. Figure 3.4 contains the Venn Diagrams representing the superposition of error loci among the three methods; Figure 3.4-A shows intersections of positions where Switch Error occurred, while Figure 3.4-B shows the intersections of positions where Error Rate positions.

As depicted in Figure 3.4-A, considering the Switch Error metric, the number of error positions exclusive of fastPHASE $(1,646)$ is roughly half of the number of error positions exclusive of Haplorec $(3,093)$, which in turn is about $75 \%$ of the number of error positions exclusive of Beagle $(4,131)$. Percentagewise, with respect to the total number of error positions for each method, those numbers represent $25 \%, 38 \%$, and $46 \%$ of the total number of error positions for each respective method. In general, the results showed that $58 \%(8,870)$ of all $(15,249)$ Switch Error positions are exclusive of one of the methods.

The Venn Diagram in Figure 3.4-B shows that 59\% $(90,186)$ of all $(153,455)$ Error Rate positions are exclusive of one of the methods. Nonetheless, differently from the Switch Error metric, the error loci considering Error Rate are more balanced among the three methods. The three methods present nearly $40 \%$ exclusive error loci, nearly $22 \%$ of errors common to one other method, and about $15 \%$ of errors in common with the other two methods.

\subsection{Discussion and Conclusion}

We have done an extensive analysis of the performance of methods PTG, Haplorec, and fastPHASE when applied to 13 large genotype datasets, with different lengths and conservation levels. The results of our analysis offer valuable insights on the behavior of those methods.

In general, we found that fastPHASE and Haplorec have somewhat similar accuracy, as indicated by Error Rate, both being more accurate than PTG, although not by a large margin. Interestingly, although fastPHASE is computationally more expensive than Haplorec, the latter 
(A) Switch Error

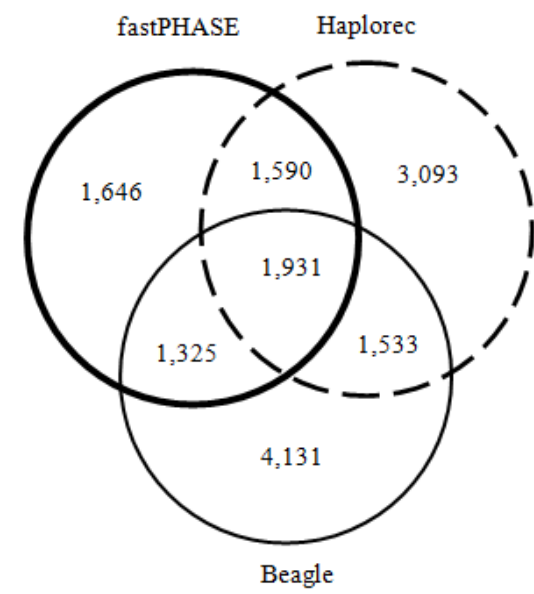

(B) Error Rate

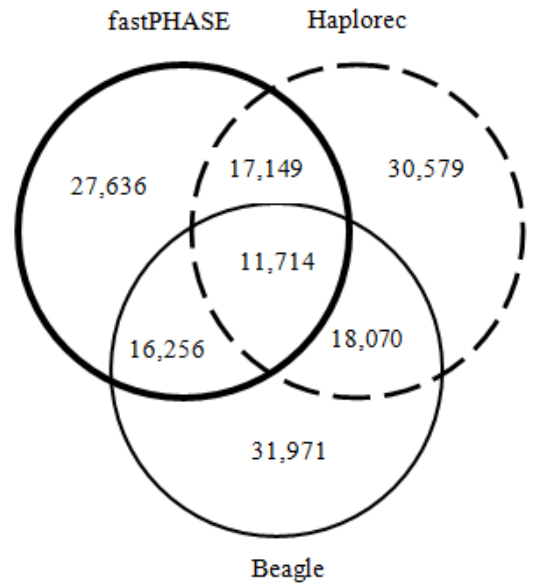

Figure 3.4: Venn diagram for the errors loci for fastPHASE, Haplorec, and Beagle, associated with Switch Error loci (A) and with Error Rate loci (B).

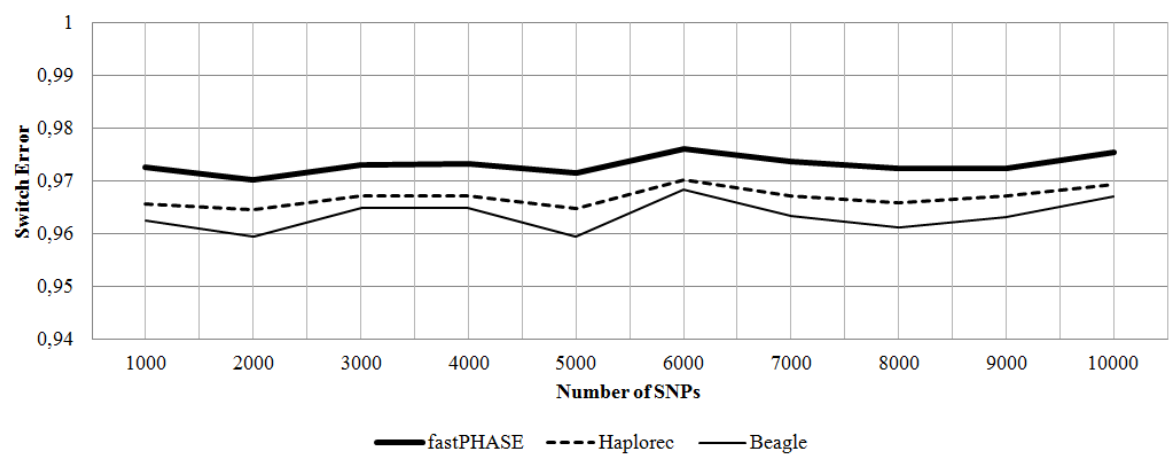

Figure 3.5: Comparison of the Switch Error scores of the three best methods analyzed, considering 100 datasets of variable number of SNPs. In the $X$ axis, the number of SNPs in the datasets; in the $Y$ axis, the average Switch Error score in 10 datasets with $X$ SNPs.

may fail to resolve long sequences. While Haplorec did not resolve our longest set of sequences (1,600 SNPs), PTG finished within 17 minutes, with Error Rate 14.2\%, and fastPHASE finished in three hours, with Error Rate $11.3 \%$. Haplorec failed to solve many genotype datasets with sequences of length larger than 1000 (experiments not shown).

When the Switch Error measure is considered, Haplorec and fastPHASE both achieve almost perfect scores (all above 0.9), while PTG reaches values below 0.7 in about half of the datasets. That can be explained by the fact that the errors made by the first two methods tend to occur together, while the errors made by PTG tend to be scattered along the sequences.

Naturally, all three methods did better with smaller and more conserved sequences, but conservation seems to particularly favor fastPHASE. We plotted a map of superposed error 
positions, and we observed that there is a relationship between LD and Error Rate. In general, for the more conserved datasets, in the more conserved regions fastPHASE and Haplorec both had superior performance, while PTG seems not to be so sensitive to sequence conservation.

We analyzed the specific sequence positions where all the methods failed, and we observed that they are relatively few, roughly $7.2 \%$ altogether, with very little deviation for each specific dataset. The sequence positions where all methods failed to solve are somewhat difficult to explain, because they represent exceptions to the biological principles used to guide the methods. No method in the literature seems to be able to foresee or deal with those cases based only on genotype data.

Finally, we also observed a strong inverse relationship between Error Rate and LD of the SNPs, although LD is not the only factor involved in the Error Rate.

We believe that the insights provided by our analysis can be used for a more effective choice of algorithms used, and can also be explored in the design of better approaches for the Haplotype Inference Problem. 


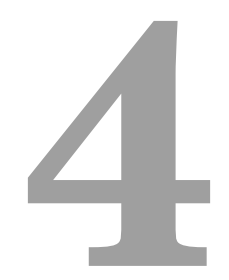

\section{A Proposed Haplotype Inference Method}

One possible way of acquiring haplotype data is to infer them from genotype data, which are highly abundant. That motivates the Haplotype Inference (HI) problem, whose computational cost depends on the evolutionary model considered. One such model is based on the biological sound Parsimony Principle, but it is proved to be NP-hard (GUSFIELD, 2002), meaning that all algorithms currently known can only solve it in time that is exponential on the number and size of the genome sequences, which is prohibitive. Several computational methods were developed aiming to find solutions that may be biologically plausible, but they usually present high computational costs. In view of real applications, the current challenge is to infer haplotypes from large scale genotyping projects. Hence, computationally efficient methods with acceptable accuracy are in great demand. In this Chapter we present a hybrid approach that combines the efficiency of Parsimonious Tree-Grow with Markov chain choices. The result is a method that is orders of magnitude faster than the known methods, delivering results of comparable accuracy.

The rest of this Chapter is organized as follows. In Section 4.1, the proposed Markov chain used by the HybHap method is presented. The HybHap approach is introduced in Section 4.2. In Section 4.3 there is a description of the datasets and how the benchmark was organized, and in Section 4.4, the results of experiments are provided to demonstrate the accuracy and efficiency of the proposed hybrid method. Finally, we present several remarks, concluding the Chapter in Section 4.5. The results of this Chapter were presented in ROSA; GUIMAR ÃES (2013). 


\subsection{Computing The Markov Chain}

The probability that a haplotype fragment will be part of a solution, considering the parsimony criterion can be efficiently estimated using a Markov chain. Given a genotype matrix $G$, with $m$ rows and $n$ columns, a Markov chain $C$ is created with $2 n+2$ states, each state representing the start $\left(C_{\text {start }}\right)$ or the end of the chain $\left(C_{\text {end }}\right)$, or a possible symbol $s(0$ or 1$)$ in the $j$-th site of a haplotype fragment, $1 \leq j \leq n,\left(C_{j}(s)\right)$. There are three types of state transitions: $\left(C_{\text {start }}, C_{1}(s)\right),\left(C_{j-1}\left(s_{1}\right), C_{j}\left(s_{2}\right)\right)$, and $\left(C_{n}(s), C_{\text {end }}\right)$.

The initial probabilities are computed as an a priori probability of symbol $s$ occurring in the first site of all the $2 m$ haplotypes to be inferred from $G(4.1)$. The absolute frequency of symbol $s$ being in the first site of the matrix is calculated according to Equation 4.2.

$$
\begin{gathered}
\left(C_{\text {start }}, C_{1}(s)\right)=A(1, s) /(2 m) \\
A(j, s)=\sum_{y=1}^{m} f_{1}(G(y, j), s),
\end{gathered}
$$

with

$$
f_{1}(x, s)= \begin{cases}2, & \text { if } x=s \\ 1, & \text { if } x=2 \\ 0, & \text { otherwise }\end{cases}
$$

The transition probabilities whose source state is not the initial state $\left(C_{\text {start }}\right)$ and the destination is not the final state $\left(C_{\text {end }}\right)$ are denoted by $\left(C_{j-1}\left(s_{1}\right), C_{j}\left(s_{2}\right)\right)$, where $2 \leq j \leq n$. These are conditional probabilities: probability of $s_{2}$ occurring in the $j$-th site of the $2 m$ haplotypes inferred from $G$, given that $s_{1}$ occurred in the $(j-1)$-th site of said set of haplotypes (Equation 4.3). That depends on the absolute frequency of haplotypes inferred with $s_{1}$ in the $(j-1)$-th site (4.1), and an estimation of the expected frequency of symbol $s_{2}$ in the $j$-th site of those same haplotypes (Equation 4.4). 


$$
\begin{gathered}
\left(C_{j-1}\left(s_{1}\right), C_{j}\left(s_{2}\right)\right)=B\left(j, s_{1}, s_{2}\right) / A\left(j-1, s_{1}\right) \\
B\left(j, s_{1}, s_{2}\right)=\sum_{y=1}^{m} f_{2}\left(G(y, j-1), G(y, j), s_{1}, s_{2}\right),
\end{gathered}
$$

with

$$
f_{2}\left(x_{1}, x_{2}, s_{1}, s_{2}\right)= \begin{cases}2, & \text { if } x_{1}=s_{1} \text { and } x_{2}=s_{2} \\ 0.5, & \text { if } x_{1}=2 \text { and } x_{2}=2 \\ 1, & \text { if } x_{1}=2 \text { and } x_{2}=s_{2} \\ 1, & \text { if } x_{1}=s_{1} \text { and } x_{2}=2 \\ 0, & \text { otherwise }\end{cases}
$$

After constructing Markov chain $C$ as described above, a tree $T$ is computed which contains the $2 m$ haplotypes that resolve $G$. The HybHap method uses the information contained in $C$ to choose more promising branches, trying to keep $T$ with the minimum possible number of branches, so as to approach an optimal solution, according to the Pure Parsimony criterion.

\subsection{The HybHap Method}

A tree $T$, which has $n+1$ layers, each one denoted by $T(j)$, is computed. A layer can have $2 m$ nodes in the worst case (maximum possible number of distinct haplotypes to be inferred). A node in layer $T(j)$ is denoted by $T_{(j, k)(s)}$, where $s$ is the node type ( 0 or 1$)$, and $k$ is the number sequence of the node in layer $j$.

A node $T_{(j, k)(s)}$ is labelled by $\left(i_{r_{1}}, i_{r_{2}}, \ldots, i_{r_{g}}\right), 1 \leq g \leq m$, which represents the genotype fragments explained by that node. The root is labelled by all genotype $\operatorname{Ids}\left(i_{1}, i_{2}, \ldots, i_{m}\right)$. For each layer $j$ of $T$, for each node in $j$, for each genotype Id $i_{r}$ in the label of the current node, if $G$, in site $j$ of genotype, has value $1(0)$, then a node of type $1(0)$ is created on the next layer connected to the current node, and it is labelled by all genotype Ids in the present node that have value $1(0)$ in site $j$. If the value in $G$ in layer $j$ and genotype Id $i_{r}$ has value 2 , then it is checked if a value 2 was previously explained for genotype $i_{r}$. If that is not the case, then site $j$ 


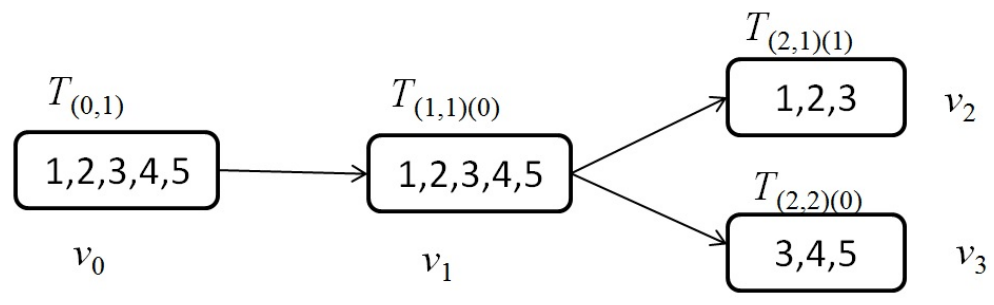

Figure 4.1: Node $v_{0}$ is the root of tree $T$; this node is in level 0 and is node number 1 of that level $\left(T_{(0,1)}\right)$. The root is labelled by five genotypes Ids $(1,2,3,4$, and 5$)$, representing all five genotypes in the input. Node $v_{1}$, denoted by $T_{(1,1)(0)}$ is in level 1 and it is the node number 1 of that level; this node is of type 0 . Node $v_{3}$, denoted by $T_{(2,2)(0)}$, is in level 2 and it is the node number 2 of that level, this node is of type 0 , and it is labelled by genotype Ids 3,4 , and 5 .

in genotype $i_{r}$ is resolved by adding two new nodes in the $(j+1)$-th layer, connected to the current node, and labelled $i_{r}$. In case a value 2 has been previously resolved, then the genotype Id and the current node are reserved to be processed after the current layer is treated. An example of tree is illustrated in Figure 4.1.

Random operations may occur in the processing of the genotypes and nodes reserved in a layer. In HybHap the Markov chain $C$ constructed will be used to decide which haplotype fragment is the most promising one. Each trace, from the root to the current node in $T$, is a valid path in $C$. There are three situations in which random choices may be needed, the others are symmetric; in those situations, new nodes are computed through $C$, and the choice is based on the maximum probability found. In case we need to choose among existing nodes to explain the reserved genotype, then we compute the Euclidean distance between the sites that have not yet been processed in the reserved genotype and all sites that have not been processed in the genotypes that are partially resolved by candidate nodes, the choice is based on the least distance. When the probabilities or distances are the same between candidate nodes, then a random choice is needed, but the chances of that actually occurring are slim.

The HybHap method (Algorithm 1) has three main steps: Initialization, Resolution of genotype prefix with known solution (genotype fragments that have only homozygous sites or one heterozygous site), as described in Algorithm 2, and explanation of genotype fragments that have no previous resolution (more than one heterozygous site), as described in Algorithm 3. In initialization the Markov chain is computed as described before, the root is created and labelled with all genotype Ids of $G$. 
Algorithm 2 explains the genotype fragments (prefix) that have at most one heterozygous site. In this case, when a site with symbol 2 is resolved for genotype $i$, we make $f(i)=$ true. All genotypes marked in the prior step $(f(i)=$ true), for a specific SNP (a layer of tree $T$ ), will be processed after all non-ambiguous genotype fragments of that layer are resolved.

In Algorithm 3, the fragments of genotypes reserved before are explained. In 2, a genotype $i$ was associated to two nodes (two is the maximum number of nodes that can explain a genotype with at least one heterozygous site). In this Algorithm 3, a Markov chain is used to decide which is the best branching option. Equation 4.5 is used, in which $P\left(v_{1}\right)$ denotes the probability that the haplotype fragment represented by node $v_{1}$ will be part of solution, according to the parsimony criterion (conservation), and $t\left(v_{1}\right)$ denotes the type of $v_{1}$. There are three cases in which the Markov chain is applied: (1) There are no branches growing from $v_{1}$ and $v_{2} ;(2)$ There is a single branch $\left(v_{1}^{\prime}, v_{2}^{\prime}\right)$ growing from each of $v_{1}$ and $v_{2}$, both of the same type $s$; and (3) There are two branches growing from $v_{1}$ but no branches growing from $v_{2}$; the other cases are symmetric. Those three situations are addressed in Algorithm 3, and illustrated in Figure 4.2-C.

\begin{tabular}{|c|c|c|c|}
\hline \multicolumn{3}{|c|}{ (A) } & \\
\hline & 1 & 2 & 3 \\
\hline & 2 & (2) & 0 \\
\hline & 2 & 2 & 2 \\
\hline & 0 & 2 & 2 \\
\hline & 0 & 0 & 2 \\
\hline & 0 & 0 & 1 \\
\hline
\end{tabular}

(B)

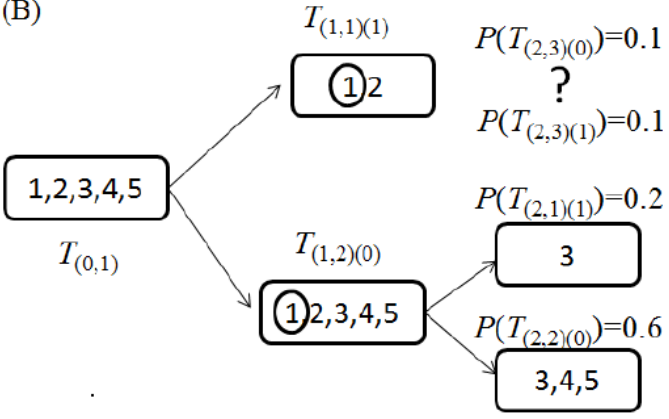

(C)

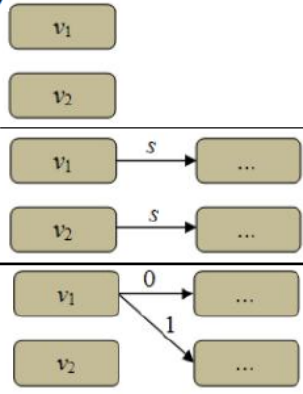

Figure 4.2: Example of Algorithm Execution

$$
P\left(v_{1}\right)\left(C_{j}\left(t\left(v_{1}\right)\right), C_{j+1}\left(s_{1}\right)\right)+P\left(v_{2}\right)\left(C_{j}\left(t\left(v_{2}\right)\right), C_{j+1}\left(s_{2}\right)\right)
$$

After building tree $T$, the final solution can be recovered by tracing from the root to each leaf of $T$, concatenating the types of the nodes in the path. The result will be the haplotype matrix that explains $G$ following the parsimony principle.

Figure 4.2-B illustrates an application of Markov chain $C$ during the construction of 
tree $T$. First the root $T_{(0,1)}$ is created and labelled by all genotype Ids of the matrix identified in Figure 4.2-A. Then the nodes descendent from $T_{(0,1)}$ are created. Since no ambiguity has been resolved in $T$ yet, nodes $T_{(1,1)(1)}$ and $T_{(1,2)(0)}$ are created to explain genotypes $(1,2)$ and $(1,2,3,4,5)$, respectively. Since in column 2 , genotypes 1 and 2 have symbol 2 , and sites of that type have been previously resolved for those genotype, their Ids are kept to be processed after all sites on the second column that do not present ambiguity or that have all previous sites without ambiguity are resolved. Hence, genotypes 3,4 , and 5 are resolved, by creating nodes $T_{(2,1)(1)}$ and $T_{(2,2)(0)}$.

After that, genotypes 1 and 2 are dealt with. There are two nodes, $T_{(2,1)(1)}$ and $T_{(2,2)(0)}$, branching from $T_{(1,2)(0)}$ that can explain genotype 1, and none from $T_{(1,1)(1)}$. In order to decide which of those nodes should be created branching from $T_{(1,1)(1)}: T_{(2,3)(0)}$ or $T_{(2,3)(1)}$. Markov chain $C$ is then used to estimate which node maximizes the probability of being also used in the resolution of other genotypes (parsimony). In the case of this example, there are the following node combinations: $P\left(T_{(2,3)(0)}+T_{(2,1)(1)}\right)=0.3$ and $P\left(T_{(2,3)(1)}+T_{(2,2)(0)}\right)=0.7$. The choice is for the option that maximizes the probability, hence, node $T_{(2,3)(1)}$ is added branching from node $T_{(1,1)(1)}$

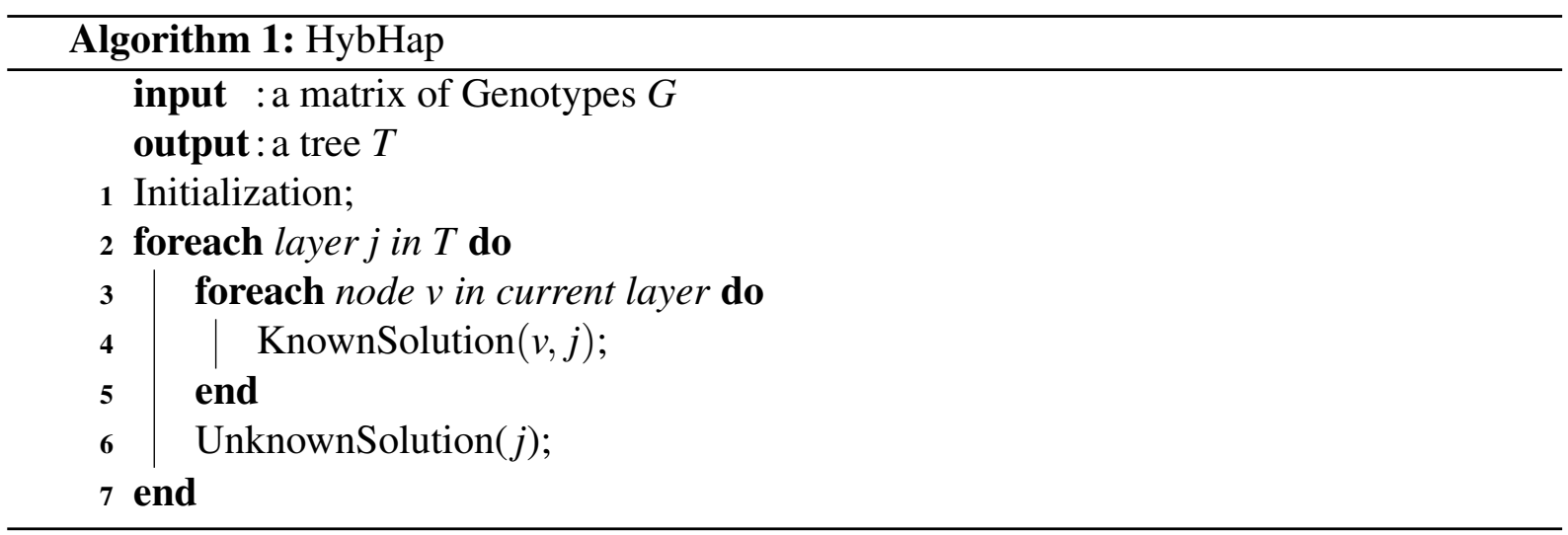

\subsection{Experiments Design}

The dataset used for the experiments was the same one used in a previous work where the performances of well known haplotype inference algorithms when dealing with data of different sizes and levels of conservation are compared (ROSA; GUIMARÃES, 2010). It is comprised by 


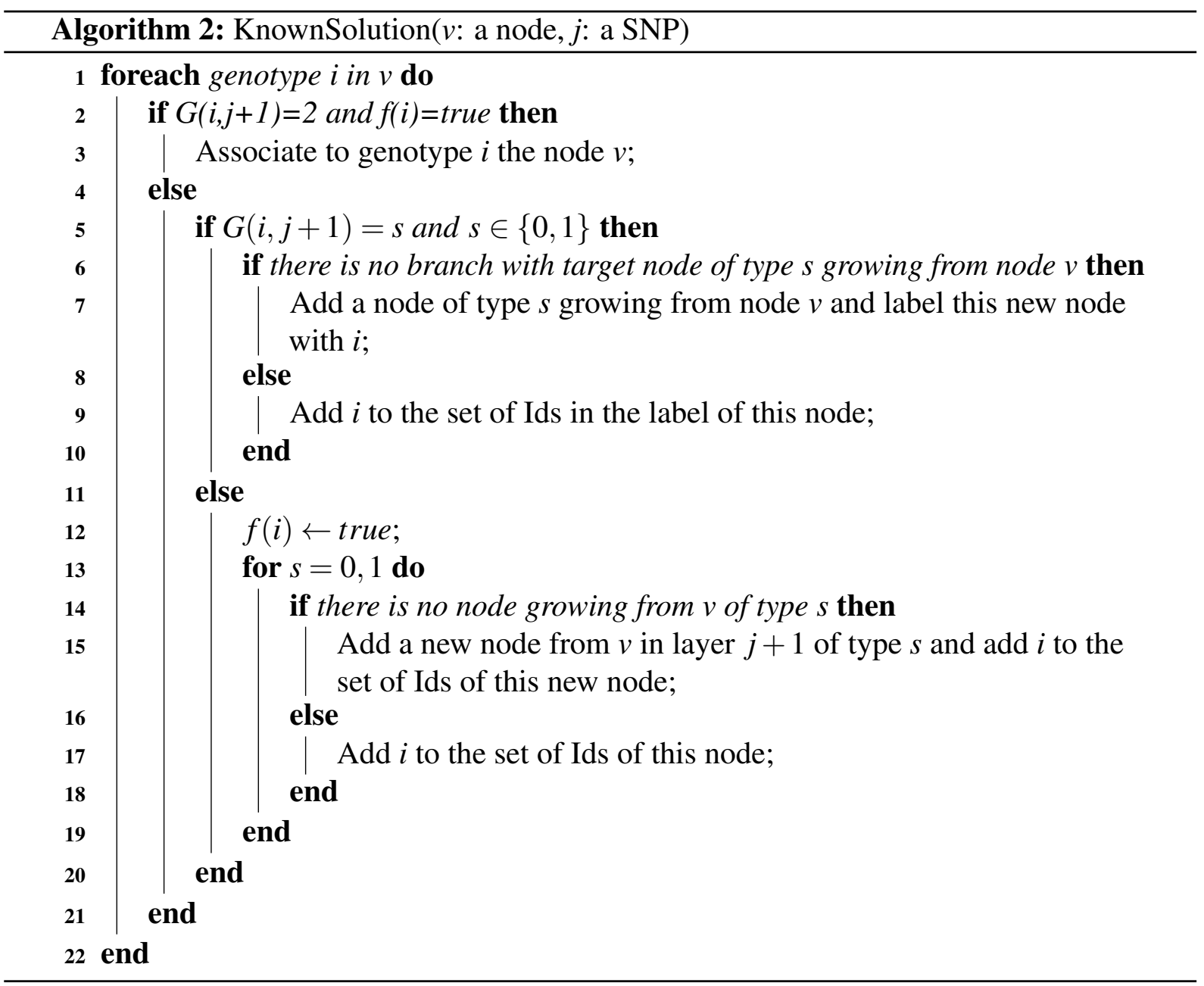

sequences originally taken from the HapMap Project (The International HapMap Consortium, 2003), which were collected from Chromosome 20 of population CEU (Caucasians resident in the state of Utah (USA) with northern European ancestry). The original dataset is composed of 13 subsets which vary in sequence length and in number of distinct haplotypes.

The set contains haplotypes of sizes 100, 200, 400, 800, 1600, and 3200 SNPs with 88 individuals, separated by size into classes A, B, C, D, E, and F, respectively. From each class, we chose randomly three instances.

The metrics used in the benchmark were Error Rate (NIU et al., 2002) and computational time. The Error Rate tells us about the capacity that one method has to correctly infer a haplotype set from a genotype set, based on a known haplotype set. The computational time is an empiric metric used to estimate computational costs; although it is not the best technique for it, in this case theoretical analysis cannot be applied to all methods.

For the comparison experiments, PTG was implemented in MATLAB 2008. Version 


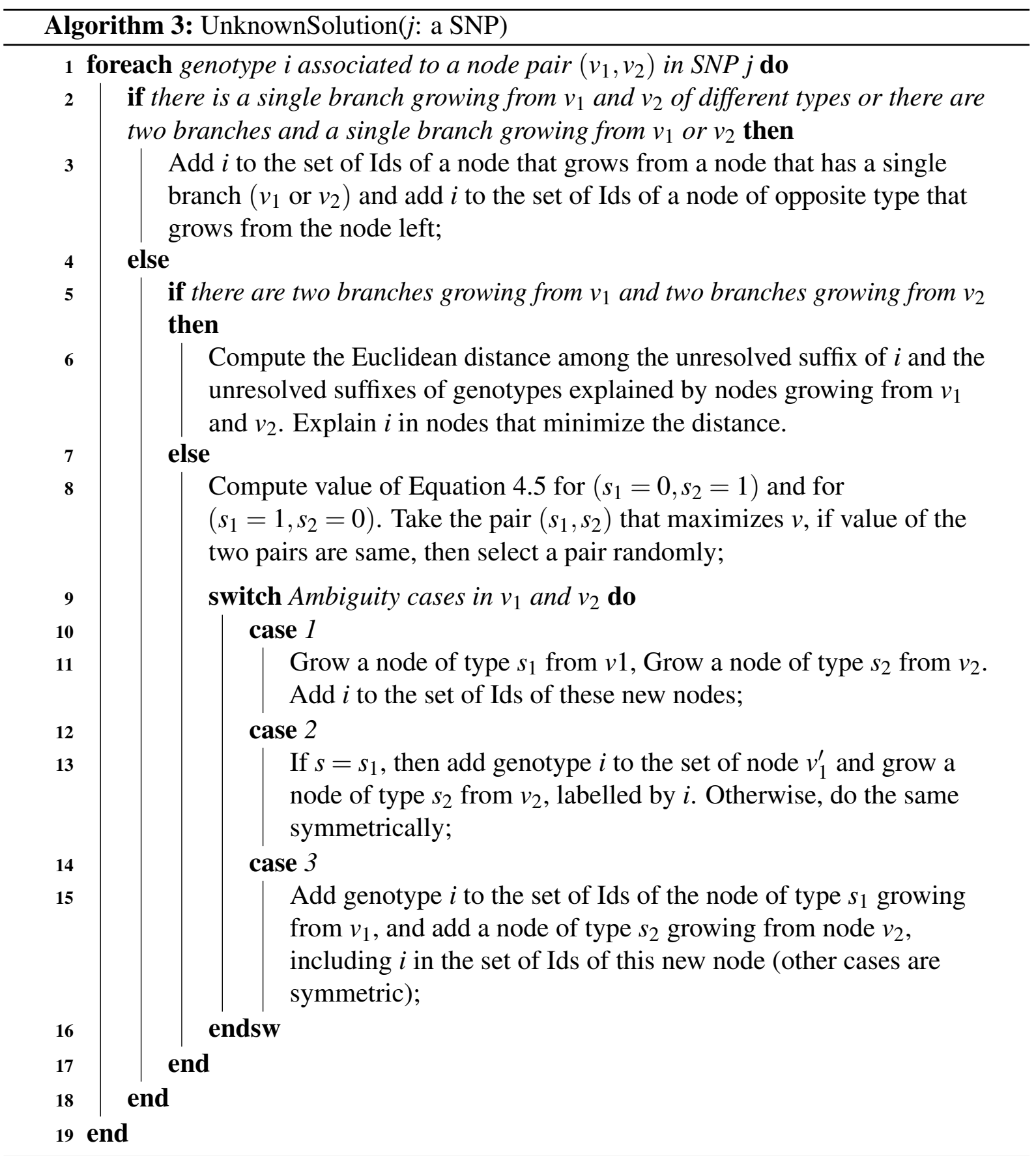



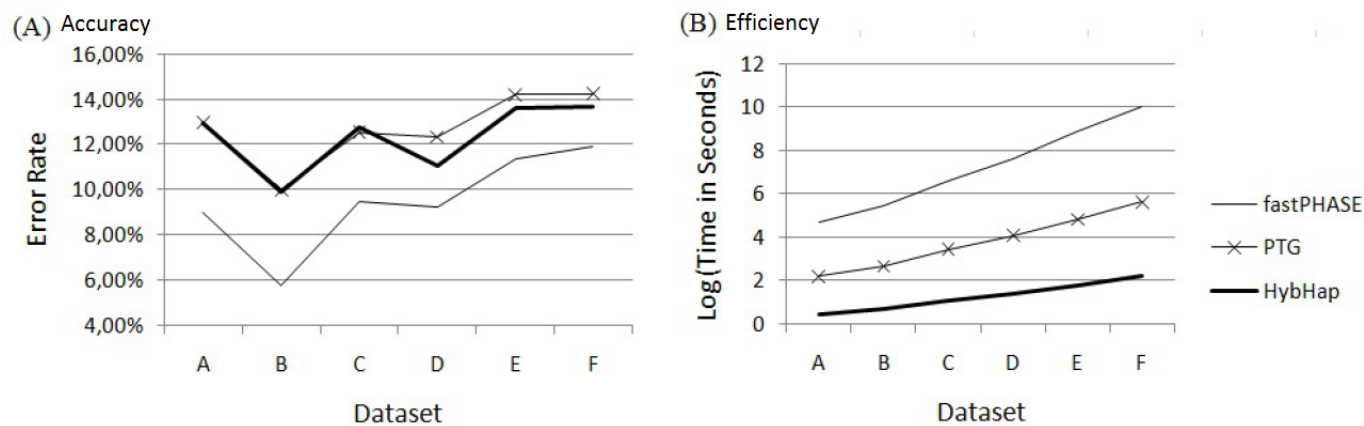

Figure 4.3: Computational Time (in seconds) and Error Rate attained in each dataset class. Each class has 88 genotypes and different number of SNPs: (A) 100, (B) 200, (C) 400, (D) 800 and (F) 1600.

1.2.3 for Windows of fastPHASE was used. The experiments ran individually in a computer with an Intel Quad Core 2.33GHz processor, with 3GB of RAM. The results are shown in Table 4.1. For each experiment, the execution time (Time) and Error Rate (ER) attained are given.

\subsection{Experiments Results}

The measures described earlier were applied to each instance. Since PTG and fastPHASE have a stochastic behavior, for comparison purposes the average over 30 executions with every single dataset was used to establish the Error Rate. Although HybHap is not a deterministic algorithm, in practice it presents standard deviation virtually equal to zero, meaning that for different executions with the same input dataset (including the same genotype order), it generates the same haplotype set.

Table 4.1: Comparison Results: Error Rate (ER) and Time in seconds (s), minutes (m) or hours (h).

\begin{tabular}{|c|c|c|c|c|c|c|}
\hline \multirow{2}{*}{ Set } & \multicolumn{2}{|c|}{ HybHap } & \multicolumn{2}{c|}{ PTG } & \multicolumn{2}{c|}{ fastPHASE } \\
\cline { 2 - 7 } & ER & Time & ER & Time & ER & Time \\
\hline A & $12.9 \%$ & $02.88 \mathrm{~s}$ & $13.5 \%$ & $53.88 \mathrm{~s}$ & $09.3 \%$ & $00 \mathrm{~h} 30 \mathrm{~m}$ \\
B & $09.9 \%$ & $04.83 \mathrm{~s}$ & $09.7 \%$ & $01.62 \mathrm{~m}$ & $05.8 \%$ & $01 \mathrm{~h} 00 \mathrm{~m}$ \\
C & $12.7 \%$ & $12.28 \mathrm{~s}$ & $12.6 \%$ & $03.73 \mathrm{~m}$ & $09.2 \%$ & $02 \mathrm{~h} 30 \mathrm{~m}$ \\
D & $11.1 \%$ & $26.11 \mathrm{~s}$ & $12.3 \%$ & $07.67 \mathrm{~m}$ & $08.7 \%$ & $05 \mathrm{~h} 24 \mathrm{~m}$ \\
E & $13.6 \%$ & $01.06 \mathrm{~m}$ & $14.2 \%$ & $16.98 \mathrm{~m}$ & $11.7 \%$ & $18 \mathrm{~h} 00 \mathrm{~m}$ \\
F & $13.7 \%$ & $03.00 \mathrm{~m}$ & $14.2 \%$ & $39.00 \mathrm{~m}$ & $11.9 \%$ & $72 \mathrm{~h} 00 \mathrm{~m}$ \\
\hline
\end{tabular}


Comparing HybHap to PTG, considering Error Rate, the accuracy of HybHap and PTG were very close, slightly favoring HybHap for the larger datasets. In all cases, HybHap was much faster than PTG. For instance, HybHap solved the largest dataset (F) in about 3 minutes, while PTG took about 39 minutes to find a less accurate solution.

Comparing HybHap to the classical approach fastPHASE, we observed that the accuracy performances considering Error Rate were very close, and for the larger datasets in the benchmark, the differences between the Error Rates for the two methods were smaller than $2 \%$. It is important to notice that, for the largest dataset, F, while HybHap needed only about 1 minute to find a solution with $13.67 \%$ of error, fastPHASE resolved this instance with $11.93 \%$ of error in about 72 hours. The difference of Error Rate in this case was $1.74 \%$, however, the time necessary for fastPHASE to resolve it was approximately 1080 times longer than the time required by HybHap.

Figure 4.3 shows graphical comparisons of HybHap with fastPHASE and PTG, in regard to Error Rate (Figure 4.3-A) and computational time (Figure 4.3-B). Since the values for computational time are so different, the values in Figure 4.3-B are depicted in log scale. It can be seen that the time of fastPHASE grows much faster than HybHap, as the length of the sequences in the datasets increases. On the other hand, while the Error Rate of HybHap is always higher than that of fastPHASE, the difference in Error Rate is virtually constant.

\subsection{Discussion and Conclusions}

In this Chapter we have proposed a hybrid method for haplotype inference. The proposed method is very stable, since in practice it presents a standard deviation of zero. In our experiments, the highest number of random operations for any instance was two, but that seldom happened. Due to that and to the efficiency of the operations in HybHap, its computational time is very low when compared to PTG and to fastPHASE.

With the enormous growth in the number of genomes available, efficient methods to deal with large datasets are highly desirable. There are many approaches to infer haplotypes with high quality, but they are applied only to small datasets, and it is not in line with the current inference requirements, which are on large scale. In face of that, HybHap presents desirable 
properties. The proposed method is computationally very efficient and in large datasets produces results with accuracy very close to that of more costly methods. That is most valuable, due to the growing number of genetic variation studies, which are performed by Computational Biology groups most of which have limited computational processing resources available.

An important point is the fact that PTG is based solely on parsimony, disregarding any other type of information or precondition about the genotype sequences, while methods based on Markov chains, such as fastPHASE, use the parsimony criterion as a help, applying additional techniques, models, and insights to find a biologically more plausible solution. Nonetheless, that combination leads to an extremely high computational time requirement. Hence, as the length of the genotype sequences grow, those methods become non-viable.

We also believe that the wide gap between the performances of HybHap and fastPHASE with respect to some cases of Error Rate is due to the fact that HybHap has no strategy to cluster together segments of different with similar characteristics regarding conservation. Since HybHap presents excellent computational cost, the original algorithm, presented in this Chapter, can be improved by strategies to associate similar regions, as it is done in fastPHASE, for instance. We are currently working on that aspect of the method. Missing data is another aspect that needs to be addressed.

The experimental analysis shows that the HybHap method is more adequate for dealing with long genome sequences. We are currently working on a theoretical argument for the fact that HybHap requires less computational time, as well as on improving its accuracy. 


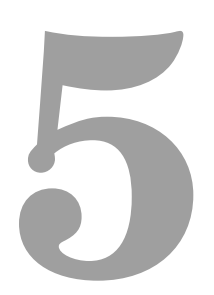

\section{Prediction of Error in Haplotype Inference}

\section{Methods through Neural Networks}

Haplotype inference (HI) methods in general have high error rates. ROSA; GUIMARÃES (2010) showed evidences that different approaches tend to fail in different parts of the sequences. That happens as a result of the different techniques applied, which are based on distinct insights and biological premisses that do not necessarily hold for all fragments of the sequences.

Characterizing scenarios for which an haplotype inference method is more likely to fail, or at least more inclined to fail than another method, may help develop more accurate haplotyping techniques. Inference error has been associated with conservation levels of the sequences. For instance, Ching and colleagues observed that in genome regions where the SNPs are highly correlated, inference methods in general tend to give more accurate solutions (CHING et al., 2002). Lack of appropriate sampling can also cause problems. More recently, (HIGASA et al., 2009) correlated LD measures with error inference in asiatic populations, probably motivated by the an insufficient number of related individuals in the sample population.

In this Chapter we analyze the power of neural networks to predict error in the results of different methods in different types of data. We develop Neural Network (NN) models to investigate the correlation of some properties of genotype datasets with the occurrence of haplotyping errors by some known inference approaches. Those models use different types of 
relevant properties of the data, collected through analysis of the genotype data. The performance of the models developed is carefully analyzed, and statistical tests show that they are highly accurate.

The rest of this Chapter is organized as follows. The next Section briefly introduces the three HI approaches considered in the study. In Section 5.2, the experiments and the data used are described. The results obtained are presented and discussed in Section 5.3. Section 5.4 contains the concluding remarks. The results of this Chapter were presented in ROSA; SANTOS; GUIMARÃES (2012).

\subsection{Haplotype Inference Methods Considered}

The inference methods considered in this analysis are fastPHASE, HaploRec, and Parsimonious Tree-Grow(PTG). fastPHASE is the version of PHASE (STEPHENS; SMITH; DONNELLY, 2001; STEPHENS; DONNELLY, 2003) for very long sequences. Just like PHASE, fastPHASE is a statistical method, based on maximum likelihood; both are very precise, but computationally highly costly. HaploRec is an approach based on Markov chains, which, although efficient and precise, fails to solve large genotype sets. PTG is a method based on pure parsimony, which resorts to random graphs to resolve difficult choices, and manages to deal with long haplotype sequences. Those methods were chosen because they resort to different computational techniques and distinct biological principles related to the haplotype inference problem.

\subsection{Experiments Design}

\subsubsection{Genotype Data Base}

The HapMap project (The International HapMap Consortium, 2003) is an invaluable source of information regarding haplotype shared among individuals of a given population; the sequences used in our experiments were collected from HapMap Phase III; they involve sequences of Chromosome 20 of nonrelated individuals of six partly mapped ethnic populations: Chinese in 
Denver (CHD), Indian in Houston (GIH), Japanese in Tokyo and Chinese in Beijing (JPT-CHB), African Luhya in Kenia (LWK), African Maasai in Kenia (MKK), and Italians in Toscana (TSI). Chromosome 20 has more than 36,000 SNPs mapped so far in HapMap Phase III. For the experiments, 1,000 of those were randomly selected in each population, in order to generate a considerable number of different scenarios. The data collected, which were sequences over the alphabet $\{\mathrm{A}, \mathrm{C}, \mathrm{G}, \mathrm{T}\}$, were pre-processed, with the most frequent allele being represented by the symbol 0 , and the one with the least frequency was represented by the symbol 1 . Those sequences were then adapted to the input formats of the methods being analyzed. The solutions generated by each method were used to construct the meta-data bases.

\subsubsection{Genotype Meta-Data Bases}

Two meta-data bases were constructed over the original set of genotypes considering the error metrics with respect to: (1) Individuals, and (2) SNPs samples, independently of the individuals. The database patterns on the Individuals base and on the SNPs base were treated separately. Each one of the meta-data bases contains the solutions provided by each of the methods studied, with their respective errors, as well as some genotypic properties to be used as inputs for the neural networks.

Although HaploRec is not a deterministic method, it usually converges in only one execution; only in rare situations that does not happen. Hence, the sample standard deviation of the error rates of the instances is always zero. On the other hand, algorithms fastPHASE and PTG present stochastic behavior, and, for each instance, it is necessary to execute them several times to obtain a stable solution. The least number $(n)$ of experiments required to obtain a stable average solution, with an standard error E of 0.0015, was computed using Equation (5.1) (MONTGOMERY; RUNGER, 2003), where $s$ denotes the sample standard deviation, $t_{\alpha}$, a Student's $t$ distribution critical value, with 29 degrees of freedom and $98 \%$ of confidence (the normality of data was verified although histograms). Over all ethnic groups, the largest value obtained for $n$ was 28. Due to that, each instance was executed 30 times for each of those two methods, and the average of the error rates was taken. 


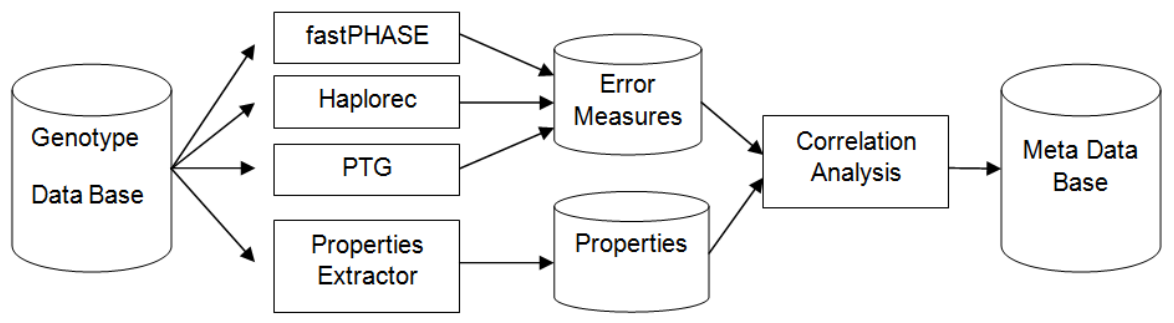

Figure 5.1: Outline of the procedure to create the Meta-Data Base

$$
n=\left(\frac{t_{\alpha} S}{E}\right)^{2}
$$

The error measurements studied were (1) Error Rate (ER) (NIU et al., 2002), the percentage of loci incorrectly inferred, determined by a direct comparison with the corresponding site in the original sequence, (2) Switch Error (SE) (LIN et al., 2002), computed by $(N-1-S D) /(N-1)$, where $N$ denotes the number of ambiguous sites, and Switch Distance (SD) (LIN et al., 2002), which represents the minimum number of fragment swaps between two inferred haplotypes needed to make them exactly equal to the original ones. For the Individuals Base, the ER and the SE measures were used as response variables, while the ER and the SD measures were used for the SNPs Base.

To determine the more appropriate input variables for the neural network models, more than a dozen biologically meaningful properties were extracted from the data, and their correlation with the error measures were carefully analyzed. From the properties considered, the ones selected to describe the individuals and the SNPS are the following. (1) Number of symbols of 0 (NS0); (2) Number of symbols 1 (NS1); (3) Heterozigocity, given by the number of symbols 2 (HTZ); (4) Number of neighbors with symbol 2 paired with symbols 2 (DHZ); (5) Number of blocks of symbols 2 (NB2); (6) average length of these blocks (LB2); and (7) Conservation level, given by the linkage disequilibrium (CSV), for SNPs, and by a Markov chain estimation, for individuals.

Figure 5.1 outlines the procedure adopted to create the Meta-data Base from the Genotype Data base. 


\subsubsection{Selecting and Validating the Neural Network Models}

$\mathrm{NN}$ models were developed to independently use each one of the 7 variables to predict each one of the 6 error measures (three methods, two error measures each).

The models were developed using the Neural Network Toolbox of the MATLAB package (MATLAB, 2011a). We designed MultiLayer Perceptron Nets, which are very powerful to approximate continuous functions (CYBENKO, 1989). Due to the reduced number of inputs and outputs, we chose to use only one intermediary layer. Numerous experiments were developed in order to determine the most appropriate configuration (topology and training parameters) for each particular network, such as number of nodes in the intermediary layer, activation function, learning algorithm, and learning rate. The number of nodes in the intermediary layer was kept low (1 to 3), in order to avoid over-fitting, and different sigmoid functions were considered before choosing the activation functions. We did experiments with three training algorithms: (1) Gradient Descent with Momentum Backpropagation (traindm), to accelerate the training process while avoiding local minima, (2) Levenberg-Marquardt Backpropagation (trainlm), which is very suitable for supervised learning, and (3) One-step Secant Backpropagation (trainoss), that converges faster than conjugate gradient methods (ZAKARIA; ISA; SUANDI, 2010).

As for stopping criteria, the maximum number of steps was used (set to 1000), as well as the maximum number of consecutive error growth for the validation (set to 5).

In order to assess the contribution of a combination of different properties, $\mathrm{NN}$ models with double inputs were designed. A total of 147 (21 input combinations x 3 methods x 2 error measures) double input neural net models were designed in a similar fashion, except that more nodes ( 1 to 5$)$ were allowed in the intermediary layer.

The effects of all combinations of those choices on the prediction accuracy were carefully analyzed.

For each run, each base was split into three disjoint sets: training (50\%), validation $(25 \%)$, and test $(25 \%)$, through randomization procedures. The validation was performed using 120 folders, applying cross-validation with reposition. That high number of folders was chosen so that a statistically relevant dataset was generated to allow for reliable hypothesis tests. 


\subsubsection{Hypothesis Tests on the Results}

All those statistical analysis were done using the Statistics Software R (R Development Core Team, 2011).

\subsubsection{Single Input Networks}

In order to assess which property (input) individually led to the design of the neural networks with the best results, hypothesis tests were performed. Let $P_{1}$ and $P_{2}$ represent the average of the errors of neural network models NNM1 and NNM2, respectivelly, given by absolute value of the difference between errors predicted by NNM1 and NNM2 (|error ${ }_{N N M 1}-$ error $\left._{N N M 2} \mid\right)$. The null hypothesis is given by: $H_{0}: P_{1} \geq P_{2}$, and the alternative hypothesis is given by $H_{a}: P_{1}<P_{2}$. The goal is to reject the null hypothesis as a way of bolstering the alternative one. Hypothesis tests were applied for all possible pairs $P_{1}$ and $P_{2}$, both in the Individuals Base and in the SNPs Base. The p-values obtained are reported in Tables 5.3 and 5.4, where lines represent $P_{1}$ and columns represent $P_{2}$. An entry represents the p-value corresponding to the test results; values below 0.005 indicate that the error for the outputs of NNM1 is statistically lower than the error for the outputs of NNM2, with a confidence level of $95 \%$.

\subsubsection{Double Input Networks}

To assess the performance of the networks designed for a combination of two biological properties (double inputs), similar hypothesis tests were performed. If $C o m b_{1}$ and $C o m b_{2}$ are different combinations of two of the 7 chosen properties, the null hypothesis is given by: $H_{0}: \operatorname{Comb}_{1} \geq \operatorname{Comb}_{2}$, and the alternative hypothesis is given by $H_{a}: \mathrm{Comb}_{1}<\mathrm{Comb}_{2}$. The p-values obtained are not all reported here. 
Table 5.1: Correlation Found Using the Individuals Properties Base.

\begin{tabular}{|c|ccc|ccc|}
\hline & \multicolumn{3}{|c|}{ Error Rate $(E R)$} & \multicolumn{3}{c|}{ Switch Error $(S E)$} \\
Variable & fastPHASE & HaploRec & PTG & fastPHASE & HaploRec & PTG \\
\hline$C S V$ & 0.555 & 0.342 & 0.558 & -0.136 & -0.253 & -0.387 \\
$N B 2$ & $\mathbf{0 . 7 9 2}$ & 0.468 & $\mathbf{0 . 8 4 8}$ & -0.185 & -0.048 & -0.271 \\
$L B 2$ & -0.097 & 0.042 & 0.010 & 0.350 & 0.395 & 0.254 \\
$D H Z$ & 0.599 & 0.359 & 0.699 & 0.001 & 0.128 & -0.144 \\
$N S 0$ & $\mathbf{- 0 . 7 7 3}$ & -0.475 & $\mathbf{- 0 . 8 6 3}$ & -0.084 & -0.114 & 0.166 \\
$N S 1$ & -0.275 & -0.139 & -0.343 & 0.174 & -0.184 & 0.022 \\
$H T Z$ & $\mathbf{0 . 8 5 8}$ & 0.513 & $\mathbf{0 . 9 7 4}$ & -0.002 & 0.194 & -0.146 \\
\hline
\end{tabular}

Table 5.2: Correlation Found Using the SNPs Properties Base.

\begin{tabular}{|c|ccc|ccc|}
\hline & \multicolumn{3}{|c|}{ Error Rate $(E R)$} & \multicolumn{3}{c|}{ Switch Distance $(S D)$} \\
Variable & fastPHASE & HaploRec & PTG & fastPHASE & HaploRec & PTG \\
\hline$C S V$ & -0.438 & -0.456 & -0418 & -0.093 & -0.395 & -0.098 \\
$N B 2$ & 0.671 & $\mathbf{0 . 7 0 7}$ & 0.650 & 0.192 & 0.861 & 0.209 \\
$L B 2$ & $\mathbf{0 . 8 2 6}$ & $\mathbf{0 . 8 5 4}$ & $\mathbf{0 . 7 7 4}$ & 0.184 & $\mathbf{0 . 7 0 8}$ & 0.192 \\
$D H Z$ & 0.483 & 0.552 & 0.472 & -0.013 & 0.629 & -0.005 \\
$N S 0$ & -0.673 & $\mathbf{- 0 . 7 1 9}$ & -0.619 & -0.102 & -0.387 & -0.101 \\
$N S 1$ & 0.638 & 0.694 & 0.623 & 0.177 & $\mathbf{0 . 7 2 9}$ & 0.186 \\
$H T Z$ & $\mathbf{0 . 7 8 3}$ & $\mathbf{0 . 8 2 1}$ & $\mathbf{0 . 7 5 2}$ & 0.220 & $\mathbf{0 . 9 2 7}$ & 0.238 \\
\hline
\end{tabular}

\subsection{Results and Discussion}

\subsubsection{Correlations between Properties and Error Measures}

The correlations obtained between the seven chosen properties and the six error measures for the three methods are reported in Tables 5.1 (Individuals Base) and 5.2 (SNPs Base).

For the Individuals Meta-data Base, the results showed that the response variable ER for methods fastPHASE and PTG presented strong evidence of correlation $r(|r|>0.7)$ with predictors NB2, NS0, and HTZ. In that base, no property presented evidence of correlation with the response variables ER and SE.

Since symbol 2 represents a DNA sequence position to be resolved by the methods, it would be expected that variable HTZ had a strong impact in the prediction results. Nonetheless, it is interesting to notice that this characteristic presents different levels of influence for the different approaches. For instance, for method PTG, the correlation between predictor variable HTZ and response variable ER was 0.974, whereas for the HaploRec method, the correlation was 

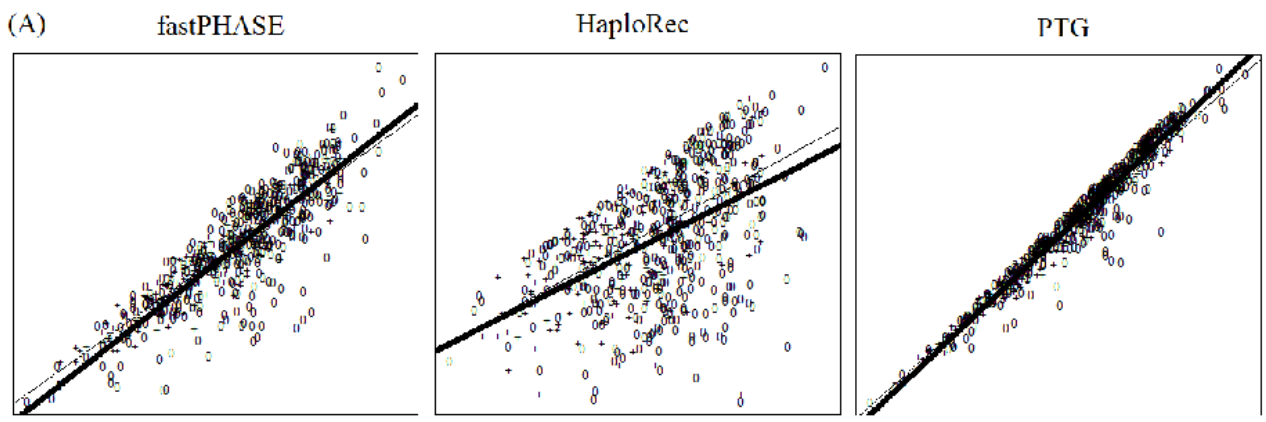

(B)
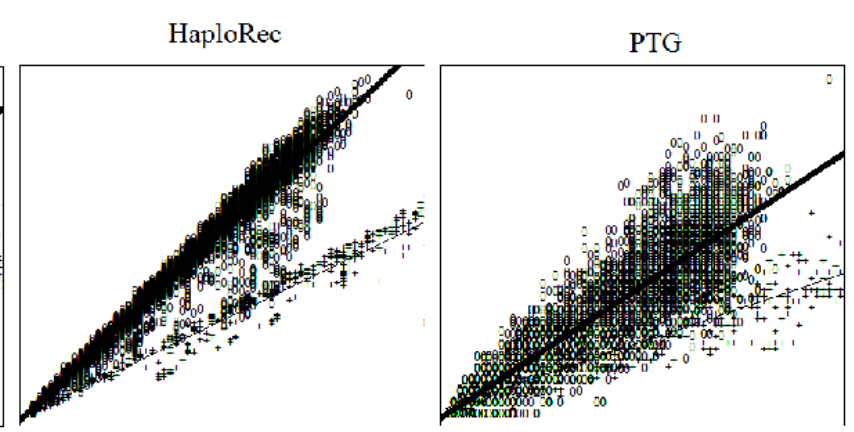

Figure 5.2: Correlation of predicting variable $H T Z$ with response variable Error Rate, $E R$, for the Individuals Base (A) and for the SNPs Base (B): In the $X$ axis, $H T Z$, and in the $Y$ axis, $E R$.

only 0.513 . None of the variables seems to have a significant correlation with the error measure Switch Error for any of the methods.

For the SNPs Meta-data Base, the most significant prediction variables for ER were LB2 and HTZ. They both presented strong correlation with the ER response variables for all methods. HaploRec also presented strong correlation with variables NS0 and NB2.

When considering the error measure SD, the only method whose results maintain some type of strong correlation with the predictor variables is HaploRec (NB2, LB2, NS0, and HTZ).

Figure 5.2 depicts the correlations of predicting variable HTZ with response variable ER in the Individuals Base and the SNPs Base. The $\mathrm{X}$ axis depicts values of HTZ, and the $\mathrm{Y}$ axis the corresponding Inference Error. The regression curve, in bold, was estimated considering all populations, except JPT-CHB. The other curve considers only the JPT-CHB population sample. In the Individuals Base, the curves present very similar coefficients, superposing in the graphics. In the SNPs base, one can clearly see the existence of distinct models, with specific angles. In this work, a decision was made not to make any type of normalization in the training data, since there is also interest in identifying and analysing situations of high discrepancy. That type of information may be useful to investigate issues, such as robustness of the methodology. 


\subsubsection{Assessment of the Neural Network Models}

Tables 5.3 and 5.4 present the p-values for the T-tests of the NN models developed for the Individuals Base, using single inputs and response variables ER and SE, respectively. In the discussion we refer to a $\mathrm{NN}$ model by the name of the predicting variable used to train it.

The analysis of the results of the experiments on the generated models for the Individuals base leads to interesting insights. For each one of the methods studied, there seems to be a different input variable that creates the best error predictor.

The NN model based on variable HTZ generates results much better than all the others for the prediction of response variable ER for Algorithm fastPHASE. On the other hand, HTZ generates the worst NN model for the same response variable for Algorithm Haplorec. A highly informative observation is that HTZ generates the best NN models for varible SE, for virtually all the algorithms studied, ironically failing only for Algorithm fastPHASE. For Algorithm Haplorec, the neural net models developed using variable NS0 defeated all the others. Variable NB2 showed to be a very good predictor for the ER measure in general. It was the best predictor for ER for Algorithm PTG, and the second best for the other algorithms. It proved to be very consistent, successfully predicting the error for all the studied approaches. Such evidence suggests that NB2 has great potential as a predictor of ER for all Algorithms.

Predicting variables CSV and LB2 were clearly non-informative for the prediction of ER, but they were very successful for the prediction of SE, with sporadic failures. For Algorithm fastPHASE, CSV is the worst variable and LB2 is the best. For Algorithm PTG, CSV is the best variable, and LB2 is the worst. That might suggest that a NN model based on a combination of these two variables could lead to much better predictions, nonetheless our results do not support that.

The results for the response variables on the SNPs Base, shown in Table 5.6 (Error Rate) and Table 5.7 (Switch Distance), were quite different. HTZ proved to be a very informative variable for NN models for both response variables, ER and SD, for almost all algorithms studied. The exceptions were variable ER for Algorithm Haplorec, and variable SD for Algorithm fastPHASE. Predicting variable NS0 showed a good performance predicting variable ER for all algorithms. Variable CSV consistently presented a very poor performance in error prediction, 
Table 5.3: Error Rate T-test's p-values of Nets with Single Input - Individuals Base

\begin{tabular}{|c|c|c|c|c|c|c|c|c|}
\hline Methods & Properties & CSV & NB2 & LB2 & DHZ & NS0 & NS1 & HTZ \\
\hline \multirow{7}{*}{ fastPHASE } & CSV & NA & 1 & 1 & 1 & 1 & 1 & 1 \\
\hline & NB2 & 0 & NA & 0 & $8.48 \mathrm{E}-194$ & $1.94 \mathrm{E}-13$ & 0 & 1 \\
\hline & LB2 & 0 & 1 & $\mathrm{NA}$ & 1 & 1 & 0.32 & 1 \\
\hline & DHZ & 0 & 1 & $5.64 \mathrm{E}-136$ & NA & 1 & $8.73 \mathrm{E}-126$ & 1 \\
\hline & NS0 & 0 & 0.99 & 0 & $3.23 \mathrm{E}-118$ & NA & 0 & 1 \\
\hline & NS1 & 0 & 1 & 0.67 & 1 & 1 & NA & 1 \\
\hline & HTZ & $\mathbf{0}$ & $3.24 \mathrm{E}-55$ & $\mathbf{0}$ & $\mathbf{0}$ & $1.05 E-117$ & $\mathbf{0}$ & NA \\
\hline \multirow{7}{*}{ Haplorec } & CSV & NA & 1 & $3.11 \mathrm{E}-59$ & 0.001 & 1 & $4.05 \mathrm{E}-54$ & 0 \\
\hline & NB2 & $1.27 \mathrm{E}-18$ & NA & $3.88 \mathrm{E}-103$ & $1.58 \mathrm{E}-27$ & 0.99 & $1.99 \mathrm{E}-104$ & 0 \\
\hline & LB2 & 1 & 1 & NA & 1 & 1 & 0.86 & 0 \\
\hline & DHZ & 0.99 & 1 & $3.03 \mathrm{E}-41$ & NA & 1 & $2.97 \mathrm{E}-40$ & 0 \\
\hline & NS0 & $8.63 \mathrm{E}-26$ & 1,03E-3 & $9.83 \mathrm{E}-125$ & $3.93 \mathrm{E}-41$ & NA & $3.79 \mathrm{E}-125$ & $\mathbf{0}$ \\
\hline & NS1 & 1 & 1 & 0,136 & 1 & 1 & NA & 0 \\
\hline & HTZ & 1 & 1 & 1 & 1 & 1 & 1 & NA \\
\hline \multirow{7}{*}{ PTG } & CSV & NA & 1 & 1 & 1 & 0.999 & 1 & 1 \\
\hline & NB2 & $\mathbf{0}$ & NA & $\mathbf{0}$ & $1.49 \mathrm{E}-126$ & $\mathbf{0}$ & $\mathbf{0}$ & $\mathbf{0}$ \\
\hline & LB2 & 0 & 1 & NA & 1 & 0 & 0,999 & 0 \\
\hline & DHZ & 0 & 1 & $1.90 \mathrm{E}-210$ & NA & 0 & $5.26 \mathrm{E}-180$ & 0 \\
\hline & NS0 & $5.82 \mathrm{E}-09$ & 1 & 1 & 1 & NA & 1 & 0.999 \\
\hline & NS1 & 0 & 1 & $1.04 \mathrm{E}-06$ & 1 & 0 & NA & 0 \\
\hline & HTZ & $1.29 \mathrm{E}-26$ & 1 & 1 & 1 & $6.58 \mathrm{E}-07$ & 1 & NA \\
\hline
\end{tabular}

always being the worst, both for variable ER and variable SD. The only exceptions were the predictions of ER for Algorithms fastPHASE and PTG, where it was better than variable DHZ, which was the worst overall. It is noticeable the good performance of variable LB2 in the prediction of the measure SD for Algorithm fastPHASE, which proved to be a very difficult problem.

A widely used approach in Computational Biology problems is to resort to different types of input data. So, a natural idea is to use multiple data to improve the quality of the predictions. It is interesting to note that the performance of the seven variables is noticeably different in regard to the two bases. While in the Individuals base different variables achieve the best performance for different Algorithms, in the SNPs base a single variable, HTZ, seems to hold information sufficient to make good predictions for both error measures. Hence, it would be expected that the prediction of errors in the Individuals base would benefit more than the SNPs base from NNs based on a combination of two or more input variables.

Table 5.5 shows the p-values obtained in a comparative analysis of the performances of the best single-input and the best double input NN models for the prediction of response variables ER and SE, in the Individuals Base. It was verified that the double-input models were 
Table 5.4: Switch Error T-test's p-values of Nets with Single Input - Individuals Base

\begin{tabular}{|c|c|c|c|c|c|c|c|c|}
\hline Methods & Properties & CSV & NB2 & LB2 & DHZ & NS0 & NS1 & HTZ \\
\hline \multirow{7}{*}{ fastPHASE } & CSV & NA & 1 & 1 & 1 & 1 & 1 & 1 \\
\hline & NB2 & 0 & NA & 1 & $3.56 \mathrm{E}-11$ & 0.986 & 0.480 & $6.71 \mathrm{E}-07$ \\
\hline & LB2 & 0 & $3.40 \mathrm{E}-19$ & NA & $4.40 \mathrm{E}-42$ & $4.43 \mathrm{E}-13$ & $6.93 \mathrm{E}-18$ & 4.39E-34 \\
\hline & DHZ & 0 & 0.999 & 1 & NA & 1 & 0.999 & 0.988 \\
\hline & NS0 & 0 & 0.016 & 0.999 & $1.51 \mathrm{E}-17$ & $\mathrm{NA}$ & 0.017 & $6.62 \mathrm{E}-12$ \\
\hline & NS1 & 0 & 0.519 & 1 & $3.99 \mathrm{E}-11$ & 0.982 & NA & $3.34 \mathrm{E}-06$ \\
\hline & HTZ & 0 & 0.999 & 1 & 0.011 & 0.999 & 0.999 & NA \\
\hline \multirow{7}{*}{ Haplorec } & CSV & NA & $1.88 E-69$ & 9.72E-15 & 1.09E-50 & $1.76 \mathrm{E}-44$ & 2.21E-45 & $2.47 \mathrm{E}-59$ \\
\hline & NB2 & 1 & NA & 1 & 0.992 & 0.999 & 0.999 & 0.976 \\
\hline & LB2 & 0.999 & $2.08 \mathrm{E}-24$ & NA & $3.74 \mathrm{E}-17$ & $5.17 \mathrm{E}-14$ & $1.30 \mathrm{E}-11$ & $5.14 \mathrm{E}-20$ \\
\hline & DHZ & 1 & 0.007 & 1 & NA & 0.790 & 0.936 & 0.274 \\
\hline & NS0 & 1 & 0.001 & 0.999 & 0.209 & NA & 0.781 & 0.079 \\
\hline & NS1 & 1 & $9.63 \mathrm{E}-05$ & 0.999 & 0.063 & 0.218 & NA & 0.011 \\
\hline & HTZ & 1 & 0.023 & 1 & 0.725 & 0.920 & 0.988 & NA \\
\hline \multirow{7}{*}{ PTG } & CSV & NA & 4.12E-32 & 0 & $1.205 E-49$ & $3.94 \mathrm{E}-56$ & $1.25 \mathrm{E}-52$ & 2.03E-36 \\
\hline & NB2 & 1 & NA & 0 & $4.11 \mathrm{E}-06$ & $2.27 \mathrm{E}-07$ & $6.06 \mathrm{E}-08$ & 0.039 \\
\hline & LB2 & 1 & 1 & NA & 1 & 1 & 1 & 1 \\
\hline & DHZ & 1 & 0.999 & 0 & NA & 0.305 & 0.140 & 0.999 \\
\hline & NS0 & 1 & 0.999 & 0 & 0.694 & NA & 0.276 & 0.999 \\
\hline & NS1 & 1 & 0.999 & 0 & 0.859 & 0.723 & NA & 0.999 \\
\hline & HTZ & 1 & 0.960 & 0 & $1.49 \mathrm{E}-05$ & $3.03 \mathrm{E}-06$ & $8.24 \mathrm{E}-07$ & NA \\
\hline
\end{tabular}

Table 5.5: Comparison of the Best Single and Double-input Models - Individuals Base

\begin{tabular}{|c|c|c|c|c|c|c|}
\hline Error & Method & Model Single Input (S) & Model Double Input (D) & $H_{a}: S<D$ & $H_{a}: S>D$ & $H_{a}: S \neq D$ \\
\hline \multirow{3}{*}{ ER } & fastPHASE & HTZ & HTZ+LB2 & 1 & $1.28 \mathrm{E}-36$ & $2.56 \mathrm{E}-36$ \\
\cline { 2 - 7 } & Haplorec & DHZ & DHZ+NS0 & 0.999 & $2.73 \mathrm{E}-05$ & $5.45 \mathrm{E}-05$ \\
\cline { 2 - 7 } & PTG & NB2 & CSV+HTZ & 1 & 0 & 0 \\
\hline \multirow{3}{*}{ SE } & fastPHASE & LB2 & CSV+NS0 & 1 & $4.46 \mathrm{E}-60$ & $3.19 \mathrm{E}-39$ \\
\cline { 2 - 7 } & Haplorec & CSV & CSV+NS0 & 1 & $2.96 \mathrm{E}-97$ & $5.92 \mathrm{E}-97$ \\
\cline { 2 - 7 } & PTG & CSV & CSV+HTZ & 0.998 & 0.001 & 0.003 \\
\hline
\end{tabular}

consistently better than the models with single input. In regard to the NN model to predict the measure SE for Algorithm PTG, although there has been an improvement, it was not statistically significant.

Since the NN models based on variable HTZ showed good performances predicting almost all error measures for algorithms on the SNPs Base, little improvement was expected in the performance of the NNs with double inputs. Nonetheless, the comparison of the best performing single-input and the best performing double-input NN models for the SNPs Base, shown in Table 5.8 show that there has been an improvement in the prediction of three (or half) of the response variables: For the NN model to predict ER for Algorithm Haplorec, and for the NN models to predict SD measures of methods fastPHASE and PTG.

For predictions of the SD response variable, there are two interesting overall observations. 
Table 5.6: Error Rate T-test's p-values of Nets with Single Input - SNPs Base

\begin{tabular}{|c|c|c|c|c|c|c|c|c|}
\hline Methods & Properties & CSV & NS0 & NS1 & HTZ & NB2 & LB2 & DHZ \\
\hline \multirow{7}{*}{ fastPHASE } & CSV & NA & 1 & 1 & 1 & 1 & 1 & 0 \\
\hline & NS0 & 0 & NA & 0 & 1 & 0 & 0 & 0 \\
\hline & NS1 & 0 & 1 & NA & 1 & 1 & 0,931 & 0 \\
\hline & HTZ & 0 & 4.30E-89 & $\mathbf{0}$ & NA & 0 & $\mathbf{0}$ & 0 \\
\hline & NB2 & 0 & 1 & 0 & 1 & NA & 0 & 0 \\
\hline & LB2 & 0 & 1 & 0.069 & 1 & 1 & NA & 0 \\
\hline & DHZ & 1 & 1 & 1 & 1 & 1 & 1 & NA \\
\hline \multirow{7}{*}{ Haplorec } & CSV & NA & 1 & 1 & 1 & 1 & 1 & 1 \\
\hline & NS0 & $\mathbf{0}$ & NA & 0 & $5.53 \mathrm{E}-81$ & 1.14E-17 & $\mathbf{0}$ & $\mathbf{0}$ \\
\hline & NS1 & 0 & 1 & NA & 1 & 1 & 1 & 0 \\
\hline & HTZ & 0 & 1 & 0 & NA & 1 & $4.06 \mathrm{E}-155$ & 0 \\
\hline & NB2 & 0 & 1 & 0 & $7.92 \mathrm{E}-33$ & NA & 0 & 0 \\
\hline & LB2 & 0 & 1 & 0 & 1 & 1 & NA & 0 \\
\hline & DHZ & 0 & 1 & 1 & 1 & 1 & 1 & NA \\
\hline \multirow{7}{*}{ PTG } & CSV & NA & 1 & 1 & 1 & 1 & 1 & $5.92 \mathrm{E}-31$ \\
\hline & NS0 & 0 & NA & 0 & 1 & $1.22 \mathrm{E}-163$ & 0 & 0 \\
\hline & NS1 & 0 & 1 & NA & 1 & 1 & 1 & 0 \\
\hline & HTZ & 0 & $1.09 \mathrm{E}-167$ & $\mathbf{0}$ & NA & $\mathbf{0}$ & 0 & 0 \\
\hline & NB2 & 0 & 1 & 0 & 1 & NA & 0 & 0 \\
\hline & LB2 & 0 & 1 & 0 & 1 & 1 & NA & 0 \\
\hline & DHZ & 1 & 1 & 1 & 1 & 1 & 1 & NA \\
\hline
\end{tabular}

(1) The combination of variables HTZ and DHZ generated a NN model better than the single variable LB2 for the prediction of the SE measure for Algorithm fastPHASE. This is the only case among all experiments with the SNPs base in which the winning combination does not involve the winner variable for single entry NN models. (2) The SD measure of all algorithms analyzed is better predicted with a combination of the same variables, HTZ and DHZ, which suggests that those two variables are strongly and directly associated with the Haplotype Inference Problem itself.

It is clear that the accuracy of the methods depends on the initial set of the inputs to the neural network, which agrees with generalized meta-learning concept for the Algorithm Selection Problem proposed by Smith-Miles (SMITH-MILES, 2009).

\subsection{Conclusions}

The work presented here consisted of the design and analysis of NN models to predict the error in the results of three different approaches for the HI problem. The study included a correlation analysis of more than a dozen genomic properties of the input sequences and the error measures (response variables) of three methods when applied to that data. That study led to 
Table 5.7: Switch Distance T-test's p-values of Nets with Single Input - SNPs Base

\begin{tabular}{|c|c|c|c|c|c|c|c|c|}
\hline Methods & Properties & CSV & NB2 & LB2 & DHZ & NS0 & NS1 & HTZ \\
\hline \multirow{7}{*}{ fastPHASE } & CSV & NA & 0.238 & 0.724 & 0.999 & 0.978 & 1 & 1 \\
\hline & NS0 & 0.762 & NA & 0.919 & 1 & 0.999 & 1 & 0.999 \\
\hline & NS1 & 0.276 & 0.081 & NA & 0.999 & 0.942 & 1 & 1 \\
\hline & HTZ & $1.03 \mathrm{E}-12$ & $8.45 \mathrm{E}-56$ & $3.49 \mathrm{E}-15$ & NA & $5.97 \mathrm{E}-15$ & 0.999 & 0.606 \\
\hline & NB2 & 0.022 & $8.75 \mathrm{E}-05$ & 0.058 & 0.999 & NA & 1 & 0.999 \\
\hline & LB2 & 3.39E-29 & 2.01E-39 & 2.97E-29 & 2.48E-06 & $6.87 \mathrm{E}-20$ & NA & 7.94E-04 \\
\hline & DHZ & $5.37 \mathrm{E}-32$ & $4.55 \mathrm{E}-12$ & $2.67 \mathrm{E}-16$ & 0.394 & $1.67 \mathrm{E}-08$ & 0.999 & NA \\
\hline \multirow{7}{*}{ Haplorec } & CSV & NA & 1 & 1 & 1 & 1 & 1 & 1 \\
\hline & NS0 & 0 & NA & 0 & 1 & 0 & 0 & 0 \\
\hline & NS1 & 0 & 1 & NA & 1 & 1 & 1 & 0 \\
\hline & HTZ & $\mathbf{0}$ & 0 & 0 & NA & $\mathbf{0}$ & $\mathbf{0}$ & $\mathbf{0}$ \\
\hline & NB2 & $\overline{0}$ & 1 & $\overline{0}$ & 1 & NA & 0 & 0 \\
\hline & LB2 & 0 & 1 & 0 & 1 & 1 & NA & 0 \\
\hline & DHZ & 0 & 1 & 1 & 1 & 1 & 1 & NA \\
\hline \multirow{7}{*}{ PTG } & CSV & NA & 0.876 & 0.999 & 0.999 & 0.981 & 0.999 & 0.993 \\
\hline & NS0 & 0.124 & NA & 0.951 & 0.999 & 0.775 & 0.999 & 0.776 \\
\hline & NS1 & $8.31 \mathrm{E}-04$ & 0.049 & NA & 0.999 & 0.159 & 0.979 & 0.199 \\
\hline & HTZ & 9.61E-15 & 2.43E-15 & 4.05E-10 & NA & 6.38E-11 & 0.002 & $6.46 \mathrm{E}-08$ \\
\hline & NB2 & 0.019 & 0.225 & 0.841 & 0.999 & NA & 0.997 & 0.546 \\
\hline & LB2 & $1.21 \mathrm{E}-05$ & $2.17 \mathrm{E}-04$ & 0.021 & 0.998 & 0.003 & NA & 0.004 \\
\hline & DHZ & 0.007 & 0.224 & 0.801 & 0.999 & 0.454 & 0.996 & NA \\
\hline
\end{tabular}

Table 5.8: Comparison of the Best Single and Double-input Models - SNPs Base

\begin{tabular}{|c|c|c|c|c|c|c|}
\hline Error & Method & Model Single Input (S) & Model Double Input (D) & $H_{a}: S<D$ & $H_{a}: S>D$ & $H_{a}: S \neq D$ \\
\hline \multirow{3}{*}{ ER } & fastPHASE & HTZ & NS1+HTZ & 0 & 1 & 0 \\
\cline { 2 - 7 } & Haplorec & NS0 & NB2+NS0 & 1 & 0 & 0 \\
\cline { 2 - 7 } & PTG & HTZ & NS0+NS1 & 1 & 1 & 0 \\
\hline \multirow{3}{*}{ SD } & fastPHASE & LB2 & HTZ+DHZ & 1 & $2.01 \mathrm{E}-25$ & $4.02 \mathrm{E}-25$ \\
\cline { 2 - 7 } & Haplorec & HTZ & HTZ+DHZ & 0 & 1 & 0 \\
\cline { 2 - 7 } & PTG & HTZ & HTZ+DHZ & 1 & $4.28 \mathrm{E}-26$ & $8.55 \mathrm{E}-26$ \\
\hline
\end{tabular}

the selection of seven predicting variables. Artificial NN models were developed based on one or two of those seven predicting variables. Due to the relatively large number of topology and training parameters, as well as the wide variation that may occur as a result of minor changes, the experiments were developed in two phases. Initially, the best neural network configurations that presented the least prediction error in the testing data were chosen. Next, the errors predicted by the NN models designed were compared to the errors of the actual HI algorithms. Finally, hypothesis tests were conducted to allow for a sound statistical analysis of the comparisons.

Some properties, such as HTZ, presented good results for virtually all scenarios, while others presented bad results for most of the methods, as did CSV. For the Individuals Base, the combination of properties two properties always generated better predicting models than any isolated variable, whereas for the SNPs Base, that was true for only half of the scenarios; 
otherwise, HTZ alone proved to be the best choice.

An important conclusion is that the combination of properties HTZ and DHZ is directly and strongly associated with the HI problem, since for the SNPs Base it was able to improve the already excellent results of variable LB2 for predicting the SD response variable for Algorithm fastPHASE, besides being the best option for predicting the SD for all methods.

Two other properties that presented significant individual results were:

- NB2, which was very consistent in predicting response variable ER for all methods in the Individuals Base, and

- NS0, that presented good results in the prediction of response variable ER for several scenarios.

Considering the bases overall, it was observed that, while for the Individuals Base, different variables led to the best predictions for the several methods, for the SNPs Base, a single variable, HTZ, seems to be sufficient to allow NN models to make good predictions of the two error measures for all the algorithms considered. Perhaps the best of all the results from this work is to show that, regardless of the algorithm or scenario, there is always a $\mathrm{NN}$ model suitable to accuratelly predict errors for all the HI methods studied.

Although the haplotype sequences that are generated by the haplotyping algorithms are sequences on the alphabet $\{0,1\}$, our models try to estimate the errors by the inference algorithms, which are real values. We are currently developing Linear Regression Models to better assess the performance of the algorithms. 


\section{6}

\section{Predicting Error in Haplotype Inference}

\section{through MLR}

ROSA; GUIMARÃES (2010) showed that, although different algorithms for HI may present similar Error Rate scores, most of those errors occur in different loci along the genotype sequence. That can be discouraging to the idea of combining several strategies in one single method, to take advantage of the distinct insights and strategies that they take to approach the problem. Identifying regions of the genotype where each method has a higher propensity to make mistakes could help improve existing methods, and could also shad some light on unfavorable scenarios, and eventually lead to an ensemble approach, based on biological properties of the sequences.

Determining which variables could be associated with inference errors of the different methods is a difficult aspect of the problem. In ROSA; SANTOS; GUIMARÃES (2012), we investigated the power of Neural Network (NN) models to predict errors, and we assessed their performance using different error measures, on different types of data. In this Chapter, we use different statistical and computational tools to assess, based on two error measures, the errors of four haplotype inference methods.

Methods Haplorec and fastPHASE were selected due to their widespread use. Beagle (BROWNING; BROWNING, 2009, 2011) was included because it is a more recent competitive 
tool. Method PTG (LI et al., 2005) was also included because it is a strongly parsimony principle-based tool.

We analyzed the behavior of those methods in regions with different Linkage Disequilibrium and Heterozygosity values. We use Statistical tests and residual analysis to verify the stability of the MLR models developed, and we present mosaics illustrating typical error situations. We also develop decision trees for each model and error measures, in order to offer insight on the most valuable properties in each specific case. Finally, we present a comparative analysis of those decision trees, which reveals a number of interesting properties.

This Chapter is organized as follows. In the next section, a preliminary assessment of correlation between Linkage Disequilibrium and Heterozygosity is presented. Section 6.2 describes the details of the design of the regression models, as well as statistical analysis of the models accuracy and stability. In Section 6.3, decision tree models are estimated, with the goal of identifying properties that are most informative in identifying the easier and harder cases for each one of the MLR models. Section 6.4 contains a brief summary of the results and the conclusion. The preliminary results of this Chapter were presented in ROSA; SANTOS; GUIMARÃES (2012). The final results are under review on journal Computational Biology and Chemistry.

\subsection{Linkage Disequilibrium versus Heterozygosity}

The dataset used in our experiments were collected from different populations in the HapMap project (The International HapMap Consortium, 2003). The dataset contain properties of sets of haplotypes /genotypes from Chromosome 20 of five different ethnic populations: Chinese in Denver (CHD), Indian in Houston (GIH), African Luhya in Kenia (LWK), African Maasai in Kenia (MKK), and Italians in Toscana (TSI). Each segment of genotype collected has 1000 SNPs. The chromosomal positions from where the sequences were taken were randomly selected (it was necessary to obtain random samples, thus statistical results to be relevant). 


\subsubsection{Selecting the more informative $L D$ window}

Genotype datasets present different LD patterns along the SNPs sequence (ERONEN; GEERTS; TOIVONEN, 2006; SCHEET; STEPHENS, 2006). Estimation of those patterns is the core of methods Haplorec and fastPHASE, which apply Markov models to create LD mosaics. While Haplorec uses that technique to estimate windows with different LDs, excluding noninformative SNPs from the inference process, fastPHASE uses it to create groups of individuals and SNPs with similar LD patterns, in order to solve those genotypes together.

Based on the knowledge that LD is central in the problem of Haplotype Inference, in this section we developed experiments to assess the influence that the different LD patterns observed in genotype datasets may have on the occurrence of errors in the Haplotype Inference methods. For that, three basic properties are collected:

1) HTZ: number of ambiguity, given by the number of symbols 2 . This property quantify the sites that HI methods should resolve;

2) LD: correlation level among one specific SNP and its neighborhood;

3) Inference error, given by the Switch Distance metric, described next.

The error metrics more frequently used for the HI Problem are Error Rate (ER) (NIU et al., 2002) and Switch Error (SE) (LIN et al., 2002). For the Error Rate, the correct haplotypes are aligned to the inferred ones, the number of mismatches is computed, and the Error Rate is given by the ratio between the number of mismatches and the total number of sites in the dataset. The Swith Error is calculated as $(N-1-S D) /(N-1)$, where $N$ denotes the number of heterozigous loci, and $S D$ (Switch Distance) denotes the minimum number of block exchanges required between the two inferred haplotypes, in order to make them identical to the original ones (for more details see (TINININI et al., 2010)). Since $S E$ is applied on genotype fragments, and here we are analysing specific SNPs, we use the intermediate metric $S D$.

A given SNP may present different correlation levels (high, low, non-existing) with different sets of neighbor SNPs. With that concern in mind, we seek to assess what would be a more informative or appropriate window width for neighbors, and also to analyze the impact, within a given window, of SNPs without statistical evidence of correlation with neighboring SNPs. In our experiments, three window width values were used: 1,5 , and 10, considering 
neighbors only to the left (upstream region), neighbors only to the right (downstream region), and neighbors from both sides (regions). We also analyzed the behavior of the models including all SNPs within the window, and including only those SNPs with evidence of correlation, according to the Exact Fisher's Test. Given a genotype matrix $H_{n}$ with $n$ SNPs, and a window of width $w$, the correlation score for a SNP $k, L D(k, w)$, is computed as follows:

1) Left window:

$$
L D(k, w)=\frac{1}{w} \sum_{i=1}^{w} \operatorname{cor}\left(H_{k}, H_{k-i}\right)
$$

2) Right window:

$$
L D(k, w)=\frac{1}{w} \sum_{i=1}^{w} \operatorname{cor}\left(H_{k}, H_{k+i}\right)
$$

3) Left-Right window:

$$
L D(k, w)=\frac{1}{2 w} \sum_{i=1}^{w} \operatorname{cor}\left(H_{k}, H_{k-i}\right)+\operatorname{cor}\left(H_{k}, H_{k+i}\right)
$$

For each SNP $H_{k-i}\left(H_{k+i}\right)$ of $\operatorname{cor}\left(H_{k}, H_{k-i}\right)\left(\operatorname{cor}\left(H_{k}, H_{k+i}\right)\right)$ when the Exact Fisher's Test, with $95 \%$ of confidence, indicates no evidence of dependency between $H_{k}$ e $\left.H_{k-i}\right)\left(H_{k}\right.$ e $\left.H_{k+i}\right)$, then this SNP $H_{k-i}\left(H_{k+i}\right)$ is discarded.

The procedures for identifying the more informative SNP window were organized as follows.

1) The LD patterns in each dataset was computed considering different window widths, including or not, SNPs without correlation evidence, as described above. For analysis the LD values were partitioned into four ranges: [0, 0.25], for low LD; $(0.25,0.5]$, for mid-low LD; $(0.5$, 0.75), for mid-high LD, and $[0.75,1.0]$, for high $L D$, thus creating LD mosaics.

2) For each population in the dataset, HTZ patterns were computed, considering the mosaics constructed in Step (1) above;

3) For each genotype dataset resolved by each of the four Haplotype Inference methods considered (fastPHASE, Haplorec, Beagle, and PTG), two error measures were computed (Switch Error/Switch Distance and Error Rate), and the error average of the four methods were 
taken, for each dataset separately;

4) For each LD mosaic, for each of the four LD ranges previously defined, some measures were taken: $h$ (total relative HTZ frequency, considering the entire dataset) and the error (relative frequency of total errors, considering the entire dataset and the four inference methods). At the end, a quality indicator given by the ratio error $/ h$ was computed.

The ratio so computed falls in one of three scenarios, with the following interpretation: (1) Values that tend to zero indicate low error concentration with respect to the HTZ frequency (or "easy to resolve" regions); (2) Values close to 1 indicate a closer proportion of error frequency considering the HTZ frequency in a given region (or "expected error occurrence" regions); and (3) Values that are strictly greater than 1, indicate a high error frequency with respect to the HTZ concentration (or "hard to resolve" regions).

Considering the Error Rate metric, for all mosaics and all LD ranges, all the ratio values tend to 1 (results not shown), a strong evidence that the LD is not related to this type of error measure.

Table 6.1 presents the results of our analysis considering all four methods, with the Switch Error metric, where each line represents the average of the results obtained with a given LD mosaic estimation, stratified by LD level. The last line presents the average ratio obtained by all mosaics estimated by LD level. Independently of the criteria (window size and direction) used, all mosaics tend to present similar behavior in regard to their response (ratio) to each particular LD concentrations region. In our experiments, all the LD mosaics presented ratios strictly greater than 1 in regions of low LD (Low column in Table 6.1), indicating that all mosaics reported the occurrence of more errors in regions of low LD. For almost all mosaics, in all LD regions, ratios called considering the Exact Fisher's Test presented values higher or equal to the corresponding ratios taking all SNPs within the window, which indicates that this is a good filter for noisy SNPs.

The last line in Table 6.1 presents the average ratio considering all mosaics developed. In regions with low $\mathrm{LD}$ level, the ratios ranged from 1.28 to 4.5 , with average 2.54 . In regions with mid-low LDs, the ratios in general are close to 1, with average of 1.03, while for the mid-high LD regions, the ratios were strictly smaller than 1 , with average of 0.54 . Regions with high LD had 
Table 6.1: Average ratios obtained by each LD mosaic for Switch Error, considering the four LD ranges. Note particularly the line for W10_LEFT_WITH_FISHER

\begin{tabular}{lcccc}
\hline Mosaic Rule - LD Range & Low & Mid-Low & Mid-High & High \\
\hline W1_LEFT_WITH_FISHER & 1.72 & 0.99 & 0.65 & 0.19 \\
W1_LEFT_WITHOUT_FISHER & 1.72 & 0.99 & 0.65 & 0.19 \\
W5_LEFT_WITH_FISHER & 3.78 & 1.14 & 0.39 & 0.16 \\
W5_LEFT_WITHOUT_FISHER & 2.92 & 0.73 & 0.28 & 0.1 \\
W10_LEFT_WITH_FISHER & $\mathbf{4 . 5}$ & $\mathbf{1 . 0 5}$ & $\mathbf{0 . 3 3}$ & $\mathbf{0 . 1 7}$ \\
W10_LEFT_WITHOUT_FISHER & 2.84 & 0.55 & 0.23 & 0.11 \\
W1_RIGHT_WITH_FISHER & 1.28 & 1.11 & 0.84 & 0.61 \\
W1_RIGHT_WITHOUT_FISHER & 1.28 & 1.11 & 0.84 & 0.61 \\
W5_RIGHT_WITH_FISHER & 2.16 & 1.15 & 0.68 & 0.55 \\
W5_RIGHT_WITHOUT_FISHER & 1.86 & 0.94 & 0.63 & 0.48 \\
W10_RIGHT_WITH_FISHER & 2.35 & 1.12 & 0.64 & 0.58 \\
W10_RIGHT_WITHOUT_FISHER & 1.81 & 0.87 & 0.59 & 0.36 \\
W1_LEFT_RIGHT_WITH_FISHER & 1.87 & 1.25 & 0.7 & 0.41 \\
W1_LEFT_RIGHT_WITHOUT_FISHER & 1.91 & 1.12 & 0.6 & 0.32 \\
W5_LEFT_RIGHT_WITH_FISHER & 3.7 & 1.33 & 0.58 & 0.27 \\
W5_LEFT_RIGHT_WITHOUT_FISHER & 2.83 & 0.98 & 0.34 & 0.13 \\
W10_LEFT_RIGHT_WITH_FISHER & 4.45 & 1.27 & 0.48 & 0.28 \\
W10_LEFT_RIGHT_WITHOUT_FISHER & 2.7 & 0.81 & 0.28 & 0.1 \\
\hline Average & 2.54 & 1.03 & 0.54 & 0.31 \\
\hline
\end{tabular}

the smallest ratios, with an average of 0.31 . We note that mosaic W10_LEFT_WITH_FISHER (line in bold) presented the best behavior, since it has the highest ratio in the low LD regions and a ratio very close to the smallest observed in high $\mathrm{LD}$ regions.

In Figure 6.1 the boxplot for the average ratios in the four LD ranges considering all mosaics are plotted. For comparison, the specific ratios obtained for model W10_LEFT_WITH_FISHER are represented by black dots. For LD levels mid-low, mid-high, and high, the narrow confidence intervals are evidence that the mosaics in general present similar behaviors, so no specific mosaic is particularly better in general. Nonetheless, in regions with low LD, the confidence interval is wider, expressing a more diverse behavior of the mosaics estimated. Note that for this LD level, mosaic W10_LEFT_WITH_FISHER had ratio 4.5, which is higher than the upper bound of the confidence interval for that level. This high ratio value constitutes evidence that Low LD regions present very strong propensity to haplotyping error.

It is important to note that all the inference methods presented similar behaviors for each LD mosaic (data not shown). In view of that, these individual analyses are not presented. 


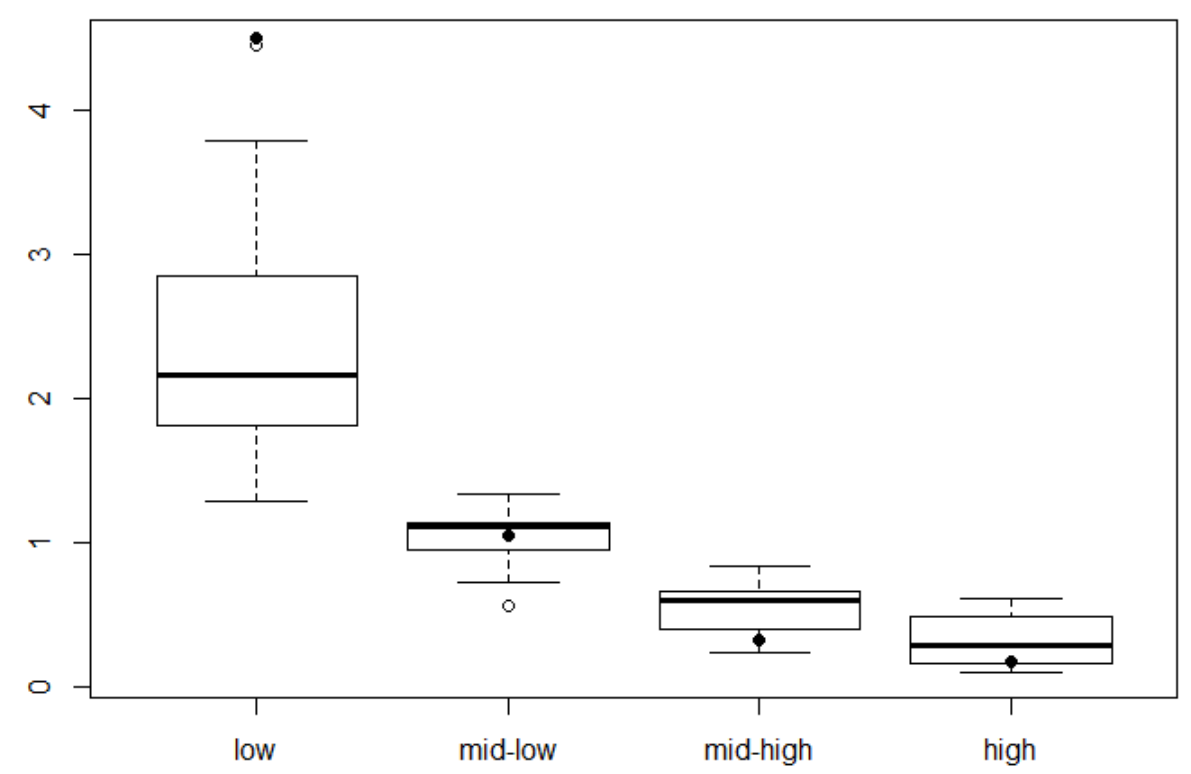

Figure 6.1: Boxplot for the average of ratios in Table 6.1. In the $X$ axis, the LD level; in the $\mathrm{Y}$ axis, the ratios. The black dots represent the ratios for mosaic W10_LEFT_WITH_FISHER.

\subsubsection{Correlation between inference error and HTZ/LD}

Table 6.2 presents the correlation obtained between inference error and HTZ/LD. The results indicate a high correlation between HTZ and inference errors in PTG, for all LD levels, which means that the errors in method PTG are highly connected to this single property; in particular, in the low LD regions, the correlation is virtually 1 . The other methods also present high correlation $(\geq 0.77)$ between HTZ and the inference error in the low LD regions, with decreasing correlation values as the LD level grows.

On the other hand, the LD value presented poor correlation with the inference error. Curiously, for all HI methods, except for PTG, the correlation was very weak or inexistent. For PTG, there was only a medium positive correlation in low LD regions and a weak inverse correlation in high LD regions.

Mosaic W10_LEFT_WITH_FISHER is used to model the LD property in the estimation of the MLR models estimated for the HI error prediction, which we describe in the next section. 
Table 6.2: Correlation between HTZ, LD and Inference Error considering LD mosaic W10_LEFT_WITH_FISHER, spread by LD level and in general.

\begin{tabular}{llccccc}
\hline Property & Method & Low & Mid-Low & Mid-High & High & General \\
\hline \multirow{6}{*}{ HTZ } & fastPHASE & 0.770 & 0.274 & 0.143 & 0.123 & 0.240 \\
& Haplorec & 0.779 & 0.293 & 0.163 & 0.100 & 0.258 \\
& Beagle & 0.771 & 0.292 & 0.122 & 0.117 & 0.250 \\
& PTG & 0.971 & 0.887 & 0.802 & 0.848 & 0.907 \\
\hline \multirow{6}{*}{ LD } & fastPHASE & 0.365 & -0.251 & -0.089 & -0.130 & -0.181 \\
& Haplorec & 0.379 & -0.267 & -0.089 & -0.114 & -0.177 \\
& Beagle & 0.380 & -0.264 & -0.076 & -0.115 & -0.172 \\
& PTG & 0.661 & 0.080 & -0.005 & -0.335 & 0.388 \\
\hline
\end{tabular}

\subsection{Multiple Linear Regression Models}

\subsubsection{Data Bases and Their Properties}

The errors of the Haplotype Inference, as well as the genotype properties were computed considering two distinct data bases: the Individuals Base and the SNPs Base. The Individuals Base is composed by properties collected across each genotype (Individual) and the SNPs Base contains properties extracted by SNP, not considering any specific individual.

For the SNPs Base the properties collected from the genotype datasets were:

(1) Heterozygosity $(H T Z)$, given by the number of symbols 2 in a given SNP;

(2) Linkage Disequilibrium $(L D)$, given by the correlation as described in Section 6.1, obtained by mosaic W10_LEFT_WITH_FISHER. The SNPs Base contains the properties and the inference errors obtained by each method for each single SNP. The error metric used for each SNP was Switch Distance $(S D)$, since in this base we did not find evidence of correlation between Error Rate $(E R)$ and the properties.

For the Individuals Base the properties collected from the genotype dataset that capture the information of ambiguity were the following:

(1) Number of symbols 0 (NSO);

(2) Number of symbols 1 (NS1);

(3) Heterozygosity $(H T Z)$, similar to SNPs Base;

(4) Density of Heterozygosity $(\mathrm{DHZ})$, represented by the number of neighbors with symbol 2 paired with symbols 2 in each individual; 
(5) Number of blocks of symbols 2 (NB2), where a block is a sequence of identical symbols in an individual;

(6) Average length of blocks of symbols 2 (LB2); and

(7) Conservation level (CSV).

Properties $N S 0$ and $N S 1$ represent the number of homozygous sites in a given Individual. $H T Z$ is given by the ambiguity level of the individual, i.e., the number of symbols 2 that must be resolved by the haplotyping method. $D H Z$ is indicated by the number of symbols 2 that are next to another 2 on the same SNP. NB2 represents the number of contiguous sequences of two or more symbols 2 in the individual. $L B 2$ is the average length of the sequences represented in $N B 2$. For a fragment of genotype $F$ with $m$ SNPs of an individual, $C S V$ is represented by probability $p$ given by Equation 6.4 computed according to ROSA; GUIMARÃES (2013).

$$
p(F)=p(F(1)) \prod_{i=2}^{m} p(F(i) \mid F(i-1))
$$

The Individuals Base has 15 attributes: The 7 properties described above, 4 results for Error Rate, and 4 results for Switch Error, one for each method considered. For each one of the eight error attributes in the Individuals Base, a MLR model was estimated and statistically validated, as described next.

\subsubsection{MLR Models}

A MLR model is composed by $p$ regressor variables $x, p$ regression coefficients $\beta$, a response variable $y$, and a noise $\varepsilon$ (Equation 6.5), where $p$ is the number of regressors. In our models, for the SNPs Base, $p=2$, and for the Individuals Base, $p=7$.

$$
y=\varepsilon+\beta_{1} x_{1}+\ldots+\beta_{p} x_{p}
$$

Twenty four MLR models were estimated, eight for the Individuals Base (four methods with two error metrics each), and sixteen for the SNPs Base (four methods with four LD levels each). The Step-Wise method (MONTGOMERY; RUNGER, 2003) was applied to select among the explanatory variables (property attributes) a suitable model for each one of the response 
Table 6.3: MLR models estimated for Individuals Base using the Step-Wise Method.

\begin{tabular}{lc}
\hline Method & Model \\
\hline ER-fastPHASE & $3.214 \times 10^{-3}+2.078 \times 10^{-6} C S V-3.499 \times 10^{-6} N S 0-$ \\
& $3.696 \times 10^{-6} N S 1$ \\
ER-Haplorec & $2.776 \times 10^{-3}+2.559 \times 10^{-6} C S V-3.046 \times 10^{-6} N S 0-$ \\
& $2.712 \times 10^{-6} N S 1$ \\
ER-PTG & $4.619 \times 10^{-3}+1.173 \times 10^{-6} C S V-6.689 \times 10^{-7} N B 2+$ \\
& $1.112 \times 10^{-7} \mathrm{DHZ}-4.716 \times 10^{-6} N S 0-5.157 \times 10^{-6} N S 1$ \\
ER-Beagle & $3.985 \times 10^{-3}+2.731 \times 10^{-6} \mathrm{CSV}-4.020 \times 10^{-6} N S 0-$ \\
& $5.564 \times 10^{-6} N S 1$ \\
$S E$-fastPHASE & $1.150 \times 10^{-2}-3.456 \times 10^{-6} C S V-1.072 \times 10^{-6} N B 2-$ \\
& $1.731 \times 10^{-6} N S 0-1.458 \times 10^{-6} N S 1$ \\
$S E$-Haplorec & $1.226 \times 10^{-2}-3.384 \times 10^{-6} C S V-2.292 \times 10^{-6} N B 2-$ \\
& $2.626 \times 10^{-6} N S 0-2.493 \times 10^{-6} N S 1$ \\
$S E$-PTG & $8.798 \times 10^{-3}-6.357 \times 10^{-6} C S V-3.129 \times 10^{-6} N B 2-$ \\
& $2.752 \times 10^{-4} L B 2-2.321 \times 10^{-6} N S 0$ \\
$S E$-Beagle & $1.225 \times 10^{-2}-4.237 \times 10^{-6} C S V-2.753 \times 10^{-6} N B 2-$ \\
& $2.379 \times 10^{-6} N S 0-2.615 \times 10^{-6} N S 1$ \\
\hline
\end{tabular}

variables (error attributes).

The training and test schemes were done with the following steps. (1) $15 \%$ of the samples for each population was randomly selected for testing, and the remaining samples were used for training; (2) For each answer variable, a MLR model was estimated using the training dataset; (3) The models constructed in Step (2) were used to predict the errors in the test data; (4) Steps (1) through (3) were repeated 120 times, in order to generate a set of predicted samples large enough to execute t-tests with confidence greater than 95\%; (5) Hypothesis tests were done with paired samples. All those tasks were done using the Statistics Software R (R Development Core Team, 2011).

\subsubsection{MLR Models with Selected Regressors}

The results of Step-Wise and the coefficients of estimated models for the Individuals Base are shown in Table 6.3. Our results show that for this Base, the most important properties were $C S V$ and NSO, selected for all estimated models. The second most important property was $N S 1$, which was considered for all models except $S E$-PTG. $D H Z$ and $L B 2$ were selected for one model each, ER-PTG and SE-PTG, respectively. HTZ was not selected for any model.

Table 6.4 presents the MLR models estimated for the SNPs Base, considering the four different LD levels and the regressors selected by the Step-Wise Method. Properties HTZ and 
$L D$ were selected for most models, expect in four situations: $S D$-PTG in Low, Mid-High and High LD selected only $H T Z$, and $S D$-Haplorec in High LD selected only $L D$. This observation give us evidences that PTG accuracy depends only on the distribution of ambiguous sites in the dataset (expressed by $H T Z$ ).

The coefficients (contribution) of $H T Z$ were positive in all models, indicating that there is a positive correlation between this property and inference error, corroborating with what was shown in Table 6.2. $L D$ presented negative coefficients in all models in all LD Levels, however in a smaller degree. The results show that as the LD level increases, the LD coefficients goes down. (For example, in model $S D$-fastPHASE for Low LD, variable $L D$ has coefficient -10.6741 , while in the equivalent model for High LD, that coefficient is -0.555609.) A similar behavior is observed for $H T Z$.

An important observation is that the noises (indicated by the intercept) are greater for Mid-Low LD than for the other LD levels. That characterizes these regions as a "hard scenario" for predicting errors in Haplotype Inference methods, which is consistent with the average ratio 1.05 found for the W10_LEFT_WITH_FISHER mosaic for that Mid-Low LD level (See Table $6.1)$.

Table 6.4: MLR models estimated for the SNPs Base considering different LD levels, using properties selected by the Step-Wise Method.

\begin{tabular}{llc}
\hline LD Level & Method & Model \\
\hline \multirow{4}{*}{ Low } & SD-fastPHASE & $0.09489+0.27096 H T Z-10.69741 L D$ \\
& SD-Haplorec & $0.08863+0.27495 H T Z-10.31239 L D$ \\
& SD-Beagle & $0.0658+0.3054 H T Z-11.1342 L D$ \\
& SD-PTG & $0.01473+0.47675 H T Z$ \\
\hline \multirow{4}{*}{ Mid-Low } & SD-fastPHASE & $3.11218+0.04988 H T Z-8.88078 L D$ \\
& SD-Haplorec & $3.687+0.059 H T Z-10.489 L D$ \\
& SD-Beagle & $3.80283+0.06224 H T Z-10.96425 L D$ \\
Mid-High & SD-PTG & $1.5385+0.4777 H T Z-4.6601 L D$ \\
& SD-fastPHASE & $0.81137+0.01172 H T Z-1.29133 L D$ \\
& SD-Haplorec & $0.82697+0.01381 H T Z-1.32023 L D$ \\
& SD-Beagle & $0.85593+0.01031 H T Z-1.13952 L D$ \\
High & SD-PTG & $0.04473+0.44419 H T Z$ \\
& SD-fastPHASE & $0.547079+0.002636 H T Z-0.555609 L D$ \\
& SD-Haplorec & $0.8345-0.7424 L D$ \\
& SD-Beagle & $0.718449+0.003674 H T Z-0.650552 L D$ \\
& SD-PTG & $0.3253+0.4363 H T Z$ \\
\hline
\end{tabular}




\subsubsection{MLR Models Accuracy}

The accuracy of the HI error prediction models was analyzed for each one of the MLR models estimated. A set of hypothesis tests was designed to assess the closeness between the values predicted $(P)$ by each MLR model and the corresponding actual $(A)$ response values of the HI method. Since there is no t-test for alternative hypothesis $A=P$, we apply the three t-tests below, none of which had the null hypothesis rejected. The three Null $\left(H_{0}\right)$ and Alternative Hypothesis $\left(H_{a}\right)$ were: (1) $H_{0}: A \geq P, H_{a}: A<P$; (2) $H_{0}: A \leq P, H_{a}: A>P$; and (3) $H_{0}: A=P$, $H_{a}: A \neq P$. These t-tests had $95 \%$ of significance.

The p-values for these three hypothesis tests are presented in Table 6.5. Note that they are all very close to 0.5 , ranging from 0.4037 to 0.5585 (hence much higher than 0.05 ). Since the null hypothesis cannot be rejected, the p-values are high, and the number of samples is sufficiently large, there is evidence that the actual values are statistically equal to the corresponding average predicted value.

The overall quality of the MLR models proposed was assessed through Residual Analysis. Figure 6.2 presents the evaluations of the best inference models, ER-Beagle (A) and SE-Beagle (B), both in the Individuals Base. For each model, one can see, on the left, the Residual plot, with the predicted values in the $\mathrm{X}$ axis and the residuals in the $\mathrm{Y}$ axis; the results very close to the ideal horizontal line on $\mathrm{Y}=0$, indicate absence of correlation between the error and the predicted values. The Quantile-Quantile $(\mathrm{Q}-\mathrm{Q})$ plots on the right show the distribution of the errors against the data, which display an almost perfect behavior along the 45-degree line for all methods, specially for Beagle. Since there is no correlation between the predicted values and the residuals, and the errors are normally distributed, we can conclude that the MLR models are stable.

Table 6.6 presents an assessment of the average relative error of the estimated MLR models. Special attention should be given to the models estimated for the Individual Base with the Switch Error metric, which are nearly perfect for all four methods. Virtually all prediction errors were smaller than $10 \%$, and the medians show that the actual errors are indeed much lower. For the models estimated with the Error Rate, methods fastPHASE and PTG had very good accuracy, while models $E R$-Haplorec and $E R$-Beagle had average performance. 
Table 6.5: Hypothesis test's p-values (with more than $95 \%$ of confidence) when comparing the average of actual values $(A)$ with the predicted ones $(P)$. The hypothesis tests are shown in the header of each column. Observe that all p-values are between 0.4037 and 0.5585 .

\begin{tabular}{|c|c|c|c|c|}
\hline Base & Method & $\begin{array}{l}\mathbf{H}_{\mathbf{0}}: \mathbf{P}>\mathbf{A} \\
\mathbf{H}_{\mathbf{a}}: \mathbf{A}<\mathbf{P}\end{array}$ & $\begin{array}{l}\mathbf{H}_{\mathbf{0}}: \mathbf{P}<\mathbf{A} \\
\mathbf{H}_{\mathbf{a}}: \mathbf{A}>\mathbf{P}\end{array}$ & $\begin{array}{l}\mathbf{H}_{\mathbf{0}}: \mathbf{P}=\mathbf{A} \\
\mathbf{H}_{\mathbf{a}}: \mathbf{A} \neq \mathbf{P}\end{array}$ \\
\hline \multirow{8}{*}{ Individual } & $E R$-fastPHASE & 0.5329 & 0.4671 & 0.4772 \\
\hline & $E R$-Haplorec & 0.4657 & 0.5343 & 0.5056 \\
\hline & $E R$-PTG & 0.4873 & 0.5127 & 0.4724 \\
\hline & $E R$-Beagle & 0.4714 & 0.5287 & 0.4670 \\
\hline & $S E$-fastPHASE & 0.5382 & 0.4618 & 0.4773 \\
\hline & $S E$-Haplorec & 0.5383 & 0.4617 & 0.4808 \\
\hline & $S E-\mathrm{PTG}$ & 0.5062 & 0.4938 & 0.4433 \\
\hline & $E D$-Beagle & 0.5456 & 0.4544 & 0.4724 \\
\hline \multirow{4}{*}{ SNP Low LD } & SD-fastPHASE & 0.5063 & 0.4938 & 0.4110 \\
\hline & SD-Haplorec & 0.4414 & 0.5585 & 0.4427 \\
\hline & SD-Beagle & 0.5154 & 0.4846 & 0.4510 \\
\hline & SD-PTG & 0.4940 & 0.5060 & 0.4701 \\
\hline \multirow{4}{*}{ SNP Mid-Low LD } & SD-fastPHASE & 0.4633 & 0.5367 & 0.4037 \\
\hline & SD-Haplorec & 0.4903 & 0.5096 & 0.4686 \\
\hline & SD-Beagle & 0.5556 & 0.4444 & 0.4369 \\
\hline & SD-PTG & 0.5264 & 0.4736 & 0.4902 \\
\hline \multirow{4}{*}{ SNP Mid-High LD } & SD-fastPHASE & 0.4840 & 0.5160 & 0.4676 \\
\hline & SD-Haplorec & 0.4613 & 0.5387 & 0.4476 \\
\hline & SD-Beagle & 0.5134 & 0.4865 & 0.4838 \\
\hline & SD-PTG & 0.5300 & 0.4699 & 0.4576 \\
\hline \multirow{4}{*}{ SNP High LD } & SD-fastPHASE & 0.4323 & 0.5676 & 0.4245 \\
\hline & SD-Haplorec & 0.4681 & 0.5319 & 0.4880 \\
\hline & SD-Beagle & 0.4663 & 0.5336 & 0.4634 \\
\hline & SD-PTG & 0.5314 & 0.4685 & 0.4607 \\
\hline
\end{tabular}




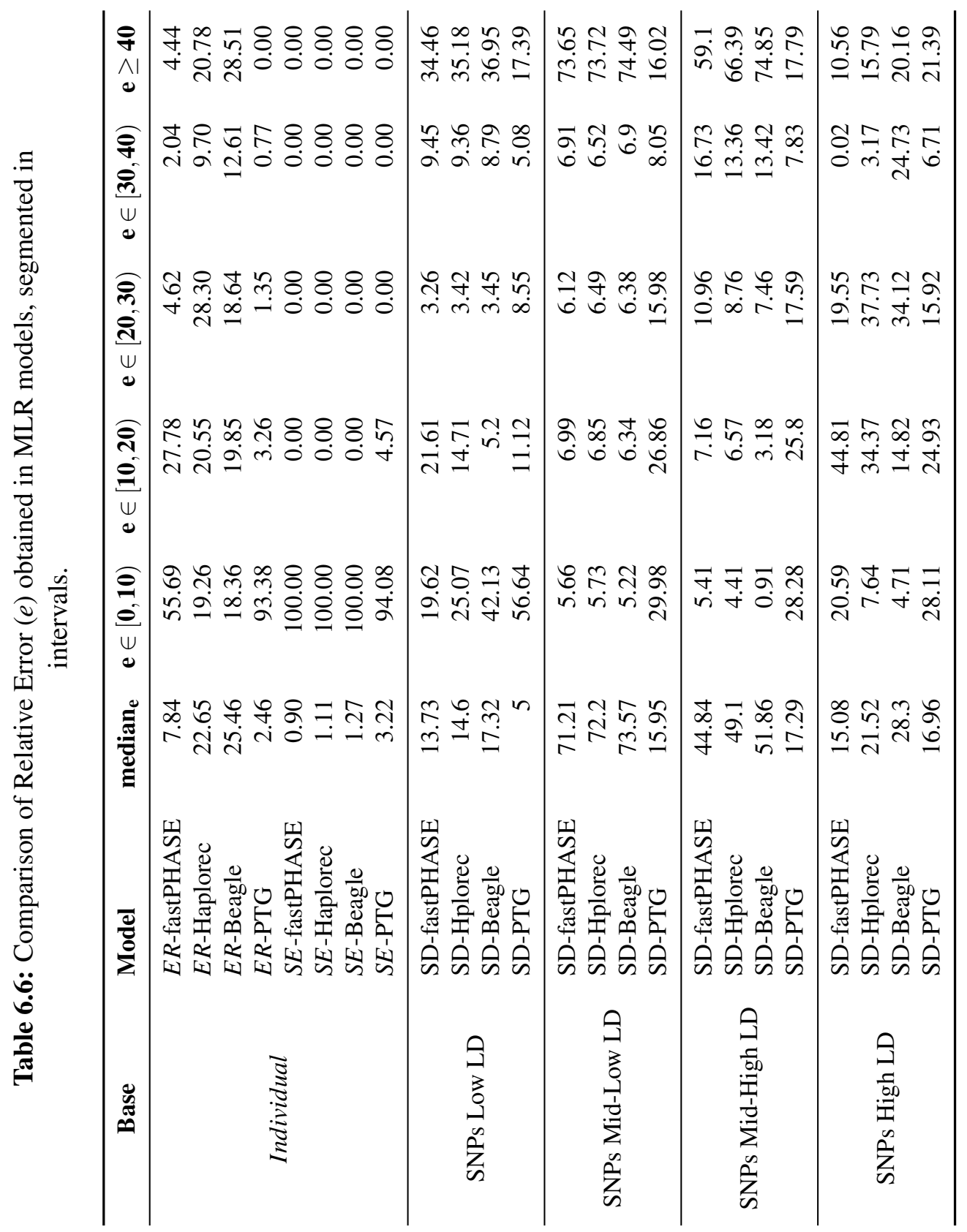



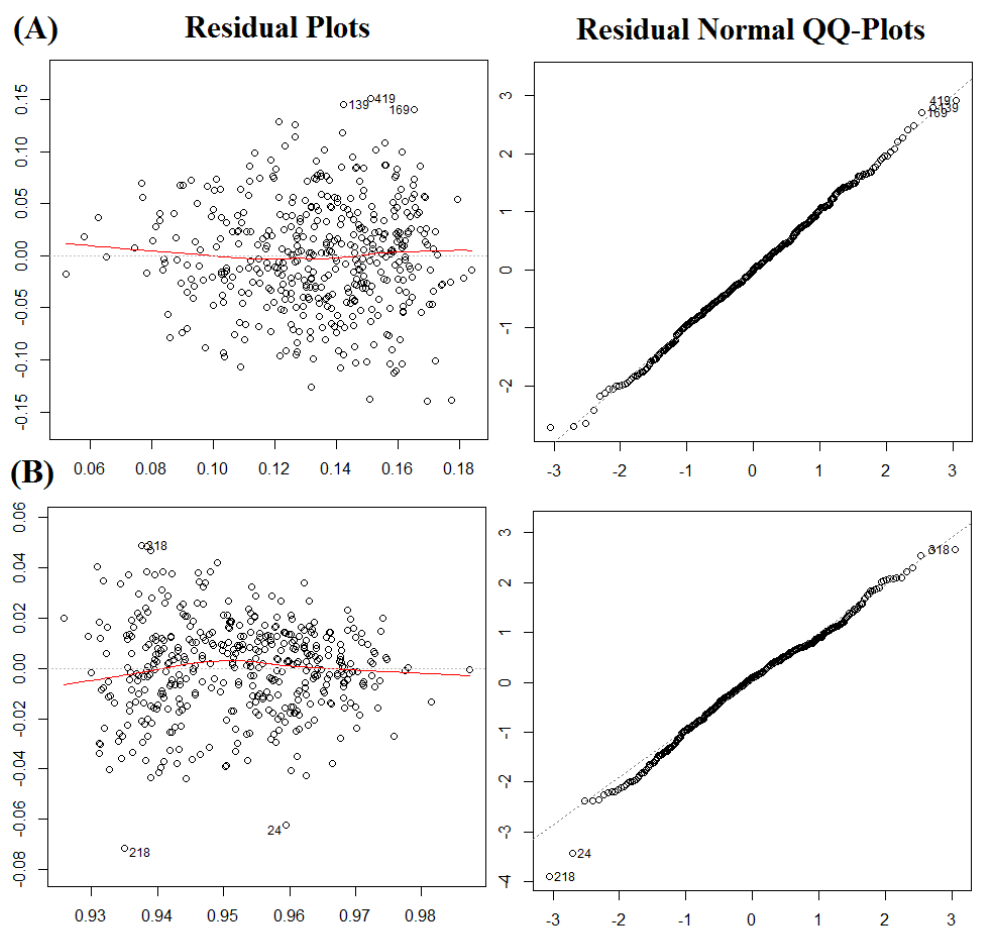

Figure 6.2: Evaluation of the MLR models proposed for the Error Rate (A) and Switch Error (B) of method Beagle, in the Individuals Base. For each model, one can see, on the left, Residual plot, with the residuals in the $Y$ axis and the predicted values in the $X$ axis, and on the right the Quantile-Quantile (Q-Q) plot.

The models estimated for the SNPs Base had performance entirely compatible with the results in Table 6.1, with the lowest median prediction error in the Low LD regions, and the highest median prediction error for the Mid-Low regions. It is important to note that these models are more accurate than models developed without considering the different LD level regions (data not shown). It is also interesting to note the steady accuracy of the $S D$-PTG models for all four LD levels.

\subsection{Decision Trees to Identify Scenarios}

While for the SNPs Base the hard and the easy error prediction scenarios are clearly delineated, that is not the case for the Individuals Base. Hence, in an attempt to identify scenarios where the proposed models have very low or very high error measures in the Individuals Base, we analyzed the properties of the genotypes with the best and the worst 100 predictions, for each one of the MLR models. Our goal was to answer the following questions. (1) Is there a property 
(or a set of properties) that separates those two genotype groups? (2) Are those properties the same for the different haplotyping methods? (3) For which scenarios of those properties do the models fail?

\subsubsection{Design of Decision Tree Models}

Decision trees were estimated considering as stop branching condition the minimum number of samples in parent (100) and minimum number of samples in child (50). A 10-fold cross-validation was applied.

Decision tree models were used to classify the selected genotype samples. The values of each one of the seven continuous properties were mapped into one of the four classes $Q-2$, $Q-1, Q+1$, and $Q+2$, defined as a function $\operatorname{Class}(\chi)$ of the continuous value $\chi$ given by:

$$
\operatorname{Class}(\chi)= \begin{cases}Q-2, & \text { if } \chi<\mu-\sigma \\ Q-1, & \text { if } \chi \in[\mu-\sigma, \mu) \\ Q+1, & \text { if } \chi \in[\mu, \mu+\sigma) \\ Q+2, & \text { if } \chi \geq \mu+\sigma,\end{cases}
$$

where $\mu$ and $\sigma$ are the average and the standard deviation, respectively.

The decision trees were constructed using both all seven variables and only the Step-Wise variables used in the respective models. The accuracy attained by each MLR model for the Individuals Base is presented in Table 6.7.

\subsubsection{Decision Tree Models Results}

Figure 6.3 shows the decision tree estimated for model $E R$-Haplorec in the Individuals Base. The root is represented by property $D H Z$, chosen as the most valuable to separate the cases for which the model $E R$-Haplorec presented the largest errors $(M)$ from the cases for which it presented the smallest errors $(m)$. The tree shows that when the value of variable $D H Z$ is within the $Q-2$ or $Q+2$ quartile, that is in node $1,80.6 \%$ of the samples are in $M$, while only $19.4 \%$ is in $m$. When the values of variable $D H Z$ are close to the average (in $Q-1$ or $Q+1$ ), $20.6 \%$ of the sample are in $M$, while $79.4 \%$ are in $m$. 
Table 6.7: Accuracy (\%) of the decision trees constructed to the sample with the 100 smallest and 100 largest relative error inferences in the MLR models estimated for Individuals Base. In gray the quantiles that classify the smallest errors $(m)$ and in black the quantiles that classify the largest errors $(M)$. The trees estimated from variables selected using Step-Wise are identified by $(*)$; trees without $(*)$ consider all seven variables.

\begin{tabular}{lcccccc}
\hline Method & $\%$ & root & Q-2 & Q-1 & Q+1 & Q+2 \\
\hline$E R$ fastPHASE & 72.0 & NB2 & & & & \\
\hline$E R$ fastPHASE* & 68.5 & NS1 & & & & \\
\hline$E R$ Haplorec & 80.0 & DHZ & & & & \\
\hline$E R$ Haplorec* & 68.5 & CSV & & & & \\
\hline$E R$ PTG & 83.0 & CSV & & & & \\
\hline$E R$ PTG* & 83.0 & CSV & & & & \\
\hline$E R$ Beagle & 84.0 & NS1 & & & & \\
\hline$E R$ Beagle* & 84.0 & NS1 & & & & \\
\hline$S E$ fastPHASE & 72.0 & NB2 & & & \\
\hline$S E$ fastPHASE* & 72.0 & NB2 & & & \\
\hline$S E$ Haplorec & 80.0 & DHZ & & & \\
\hline$S E$ Haplorec* & 75.0 & HTZ & & & \\
\hline$S E$ PTG & 83.0 & CSV & & & \\
\hline$S E$ PTG* & 83.0 & CSV & & & \\
\hline$S E$ Beagle & 84.0 & NS1 & & & \\
\hline$S E$ Beagle* & 84.0 & NS1 & & & \\
\hline
\end{tabular}

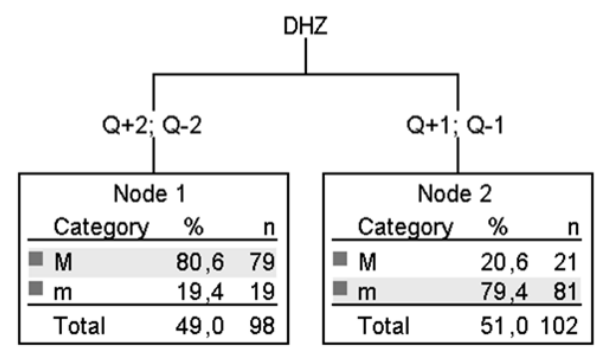

Figure 6.3: Decision Tree constructed to 100 inferences with the smallest $(m)$ and the largest $(M)$ relative error in the MLR model estimated to $E R$-Haplorec in the Individuals Base.

There was no significant difference between the accuracy of the decision trees estimated using all variables from those estimated using only the variables selected by Step-Wise. They were the same, except for models $E R$-fastPHASE, $E R$-Haplorec, and $S E$-Haplorec in the Individuals Base. Table 6.7 shows the accuracy results for the eight MLR models for both cases (all and selected variables).

Some interesting observations that we can extract from this table are: (1) All models had accuracy at least $68.5 \%$ for the Individuals Base. (2) The accuracy of all models is better for the SNPs Base than for the corresponding Individuals Base. (3) For 12 of the 16 (error measure, HI method) combination, the accuracy of the all variable model is the same as the 
model estimated with the Step-Wise selected variables. In all other cases, a different variable was chosen for the root of the decision tree, because the one selected for the all variables model had not been selected by Step-Wise. (4) The accuracy differences were relatively small, except for the $E R$-Haplorec model in the Individuals Base.

\subsection{Discussion and Conclusions}

In this work we present extensive statistical analyses of the influence that properties of the genotype sequences may have on the errors of haplotype inference methods.

We demonstrate that the correlation between LD level and the occurrence of IP errors varies along the genotypes. We also present evidence that considering a window with the 10 SNPs immediately to the left, and eliminating the non-informative SNPs through Fisher's Test is more suitable when seeking a correlation between LD and Inference Errors. It is known that eliminating non-informative SNPs is a promising strategy for haplotype inference, since it reduces noise. But, as far as we know, a quantitative assessment of the impact of that filtering on the correlation between LD and the occurrence of errors in the haplotype inference results has never been reported in the literature. The fact that SNPs in the window to the left of a SNP are apparently more informative than the SNPs on the window to the right suggests that the direction in which the methods proceed during the resolution process impacts the occurrence of errors. With that in mind, different strategies could be attempted to try to reduce the errors caused by the choice of direction in the resolution process.

We delineate scenarios, based on LD measures, that reveal higher or smaller propension of the HI methods to present inference errors. The absence of correlation between SNPs in a given region (low LD) translates into less information for the HI methods, which tend to present more errors in these regions. In this work we show that, within low LD level regions, the relative error frequency is more than four times bigger than the relative frequency of HTZ. We also show that when a SNP has LD-value between 0.25 and 0.5 , the error frequency is proportional to the HTZ level (concentration of sites to be resolved), an evidence that in those regions the LD-value is not informative, hence new alternative approaches for $\mathrm{HI}$ in these regions are necessary. For regions where LD level $>0.5$, a smaller number of errors occur, characterizing these regions as 
easy to resolve (the detailed results of the analysis are shown in Table 6.1).

Due to the correlation between LD levels and the occurrence of HI errors, specific MLR models were estimated for each particular LD interval (low, mid-low, mid-high and high), which considerably improved the prediction performance of models estimated without LD levels. We identify the Low LD regions as the easy to predict scenario, and the Mid-Low LD regions as a hard to predict scenario for MLR models based on SNPs data.

Considering individuals (genotypes) to predict inference errors showed to be far more promising than considering SNPs. Although the SNPs Base produced good results, the Individuals Base presented more accurate results in most scenarios. It is important to note that all the models based on the Switch Error metric for individuals were highly, almost perfectly, accurate. In this context, information on conservation and locality (HTZ blocks), which pertains only to individuals, seem to have considerable impact on the accuracy observed in the results, suggesting that these properties should have a more central role in the development of new techniques.

MLR models were developed based on seven biologically relevant properties, and also for properties selected using the Step-Wise method. From these models, NSO was selected as the most relevant variable, being used in all models for the Individuals base. The second most important property was NS1, used in all but one of the models. Considering only the Individuals Base, $C S V$ was selected for all models. $C V S$ tries to capture conservation information on the sequences, which is an important evidence in favor of the known biological parsimony principle. The other variables selected for the MLR models in the Individuals Base, $N S 0, N S 1, N B 2$, and $D H Z$ are indirectly related to variable $H T Z$, which was discarded by method Step-Wise from all models in this base. The experiments show statistical evidence that almost all the MLR models in Individuals can efficiently infer errors in the HI Algorithms studied, although in some specific cases high relative error and deviation measures can be observed.

Statistical tests and residual plots were shown, which show evidence of the accuracy of the MLR models estimated.

Finally, several important observations were revealed by accurate decision tree models estimated to identify properties that can separate the best $(m)$ from the worst $(M)$ scenarios in terms of inference error for each of the MLR models for those four methods. Those results point 
to different properties that can be crucial to the accuracy of specific HI methods. Since there is a correspondence between the best and the worst scenarios of the MLR models estimated for the error metrics $E R$ and $S E / S D$ for most HI methods, we claim that those variables have the same prediction power, independent of the error measure used. 


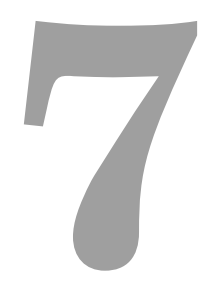

\section{A practical application}

The Human Immunodeficiency Virus (HIV) is a retrovirus that attacks the humans' CD4+ T cells, causing the decline in their natural defenses against pathogenic microorganisms. Given the high rate of mutation that retroviruses present (MANSKY, 1998), fighting them is a very difficult task. There are many variants of a same type of HIV in a single individual. This variation is even higher among viral strains from different patients. Such mutations can cause a particular patient not to have a good response to antiretroviral treatment, since the virus installed on your system may have developed resistance to some drugs used to combat the infection. Recognizing HIV mutations that lead to resistance of the virus to certain medications and predicting whether the patient will have a satisfactory response to therapy are challenges that require the use of computational and statistical techniques to be faced in a timely fashion.

Many machine learning methods have been applied in attempts to predict whether a patient will or not have a satisfactory response to HIV cocktail drugs. To this end, properties of the virus Ribonucleic Acid (RNA) sequences of each patient were considered. Neural Network models have been developed considering such characteristics (LARDER et al., 2007), Support Vector Machines models have also been introduced (ALTMANN et al., 2009; ZAZZI et al., 2012). A comparative study was conducted considering the performance of these tools and human experts (ZAZZI et al., 2011), which showed evidences that the computational methods are more efficient than clinical professionals. Methods that do not consider the genotype of the HIV have also been presented (REVELL et al., 2010; PROSPERI et al., 2010; REVELL et al., 
2013). Recently, a method based on multilabel classification exploring cross-resistence was presented (HEIDER et al., 2013).

In this work, we fitted prediction models and developed an extensive analysis involving the machine learning techniques MultiLayer Perception (MLP), Radial Basis Function (RBF), and Suport Suport Vector Machine (SVM). These approaches were selected because they are well known and they can deliver accurate results when precisely tunned. We applied Synthetic Minority Over-sampling Technique (SMOTE) (CHAWLA et al., 2002), a synthetic oversampling algorithm, to resolve the unbalance between the case and control cases without causing overfitting. In this Chapter we answer the following questions: (1) Which of those approaches has the best performance considering accuracy rate?, (2) Are there significant differences among the wrong results of each method?, (3) Are there patterns or properties of the data that induce the approaches to fail?, and (4) Mutations in RNA and clinical measures have the same predictive power on all models?

The remainder of this Chapter is organized as follows. The next section presents aspects of HIV virus and Acquired Immunodeficiency Syndrome (AIDS) treatment that will be considered in the study. In Section 7.2, the experiments and the data used are described. The results obtained are presented in Section 7.3. Section 7.4 contains a discussion of the results and our concluding remarks. The results of this Chapter were presented in ROSA et al. (2014).

\subsection{HIV and AIDS treatment}

The structure of the HIV is composed of an outer lipoprotein membrane containing specific receptors (gp120 on the surface and gp14 that crosses the membrane), an inner cover which insulates its genetic material, coding for the proteins necessary for virus replication in cell (p11 protease, integrase p31, and reverse transcriptase p51), and for two structural proteins (the capsid p24 and the nucleocapsid p17).

The cycle of HIV replication is extremely fast. Every day, about 100 copies of the virus are produced. The viral RNA has a high rate of mutations observed. The mutations occur mainly on the reverse transcriptase viral RNA replication step, since the enzyme responsible does not have the control mechanisms required to repair errors in base pairing occurring at this 
stage. These mutations produce changes in the structure of proteins, causing the virus not to be recognized by the immune system of its host, and making the new subtype resistant to the drugs used.

The parameters for evaluation of the patient state are the laboratory tests of rates of CD4+ and Viral Load (VL). According to the values obtained, anti-HIV therapy is indicated or not. In this work we applied the guideline treatment for HIV from the National Institute of Health (NIH) until February 2013, which recommended the monitoring of patients for initiation of therapy based on rate of CD4+ cells, and the patterns were as follows: (Group 1) CD4+ $<350$ cells $/ \mathrm{mL}$; (Group 2) $500 \mathrm{cells} / \mathrm{mL}>\mathrm{CD} 4+>350 \mathrm{cells} / \mathrm{mL}$; and (Group 3): CD4+ > $500 \mathrm{cells} / \mathrm{mL}$.

\subsection{Experiments Design}

\subsubsection{Data preparation}

The data used in this work were collected from the HIV Resistance Drug Database (RHEE et al., 2003). It includes 1000 patients with the following attributes: CD4+ at the beginning of the treatment (CD4), viral load at the beginning of treatment (in log base 10) (VL), RNA sequence of the Reverse Transcriptase (RT) of virus, sequence of the Protease (PR). We compute the response of the patient to treatment after 16 weeks. It is considered a positive response to the treatment when after 16 weeks there is a reduction of the viral load by a factor of at least 10 (1 in log basis). Patients with positive response are called responders (class 1$)$ and patients with negative response are called non-responders (class 0 ).

One interesting observation about this data is illustrated in Figure 7.1, the correlation between the viral load and the CD4+ cells count. In Figure 7.1 (A) are patients with CD4+ count $>350$ at the beginning of the treatment, while in Figure 7.1 (B) are counts for patients with $\mathrm{CD} 4+\leq 350$. It is expected that the larger the viral load, the least is the CD4+ cell count, since with a larger number of viruses circulating in the body fluids, represents a stronger aggression to the immunological system. Nonetheless, analyzing Figure 7.1 (A), it is easy to notice the null correlation between those two variables (line parallel to the $x$ axis), as opposed to the strong inverse correlation in Figure 7.1 (B). 
(A)

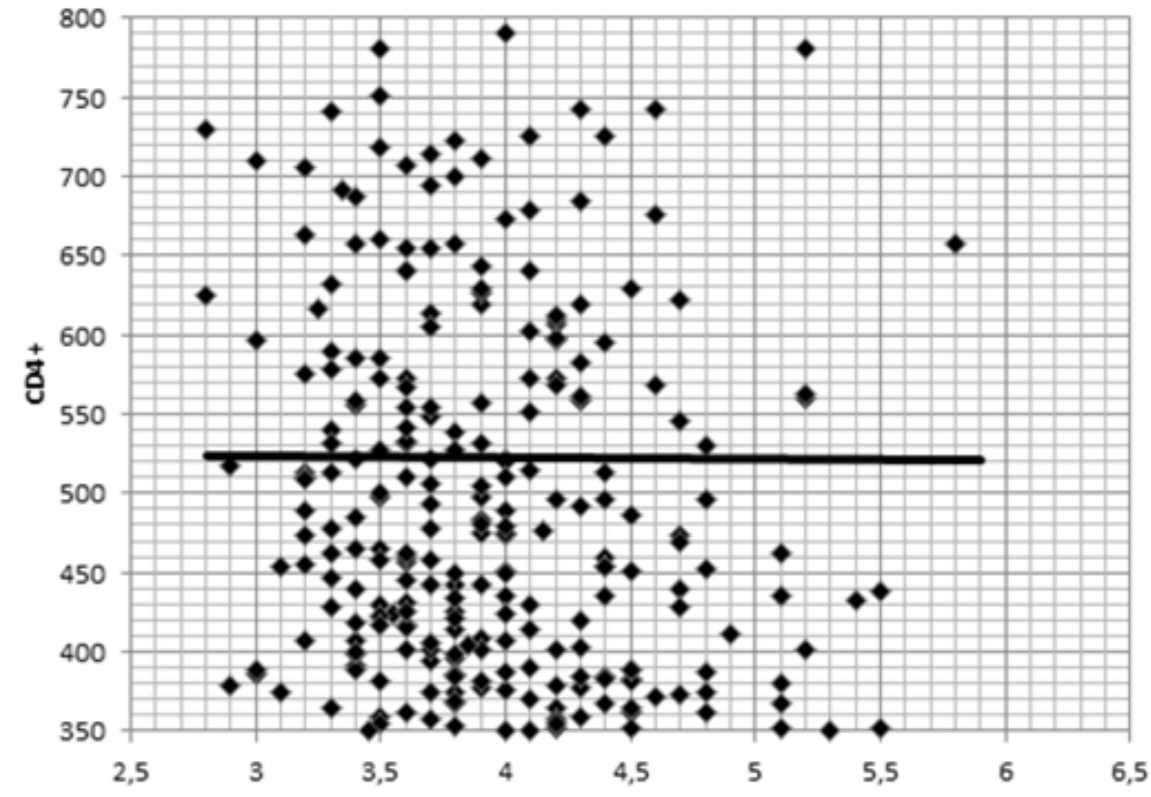

(B) Viral Load

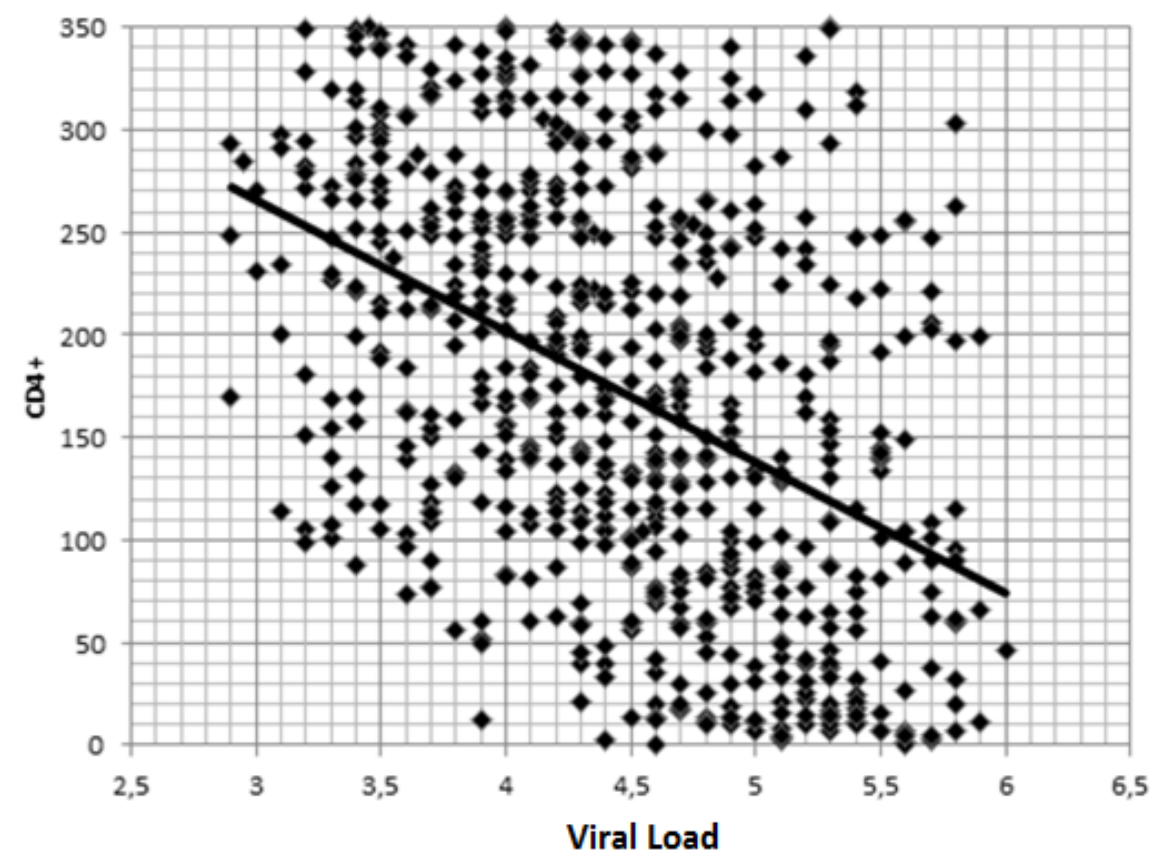

Figure 7.1: Correlation between viral load and CD4+ cells count in patients (A) with CD4+ $>350$ cells $/ \mathrm{mL}$, and (B) with CD4+ $\leq 350$ cells $/ \mathrm{mL}$.

The data presented three problems that should be dealt with before being used to train a classifier: (1) 80 of the 1000 patients do not have the PR sequence available, (2) The RT sequences have different numbers of bases, and (3) The Responders and Non-responders classes are unbalanced, there are 794 samples $(79.4 \%)$ of class 0 and 206 samples $(20.6 \%)$ of class 1.

For efficiency purposes, each amino acid was represented by a single natural number 
between 1 and 22, resulting in approximately 565 entries to the Neural Net. Since RT have different number of nitrogenous bases sequenced in each patient, the codons were translated into protein sequences, using EMBOSS Transec (RICE; LONGDEN; BLEASBY, 2000; GOUJON et al., 2010) software, and then they were aligned using the tool called Clustal Omega (SIEVERS et al., 2011; GOUJON et al., 2010). The output of Clustal Omega is composed by 26 possible symbols (22 amino acids and 4 special situations, for example, missing data and gaps). The symbols "underline" and "gap" used to represent missing data resolved the problems of (1) missing codons on RT and (2) RTs of different lengths. With the special characters inserted by the Clustal Omega, the number of inputs for the neural model was 593. A binary representation of those symbols was also considered in our experiments, but the results did not present any improvement.

The two types of approaches used to solve the problem of unbalanced classes are oversampling (replication of data from smaller class) and undersampling (data reduction of larger class). As the amount of examples for training is not large, discarding some of them would negatively affect the training; for that reason, undersampling was discarded. Simply replicating the data from minority class increases the bias of the classifier for this class, however it makes the model very specific to these replicate cases, damaging the generalization. We used the oversampling synthetic technique called SMOTE (Synthetic Minority Over-Sampling Technique) (CHAWLA et al., 2002), which creates artificial data based on spatial features between individuals of the minority class, in order to extend the decision region, thus increasing the generalization power of the classifiers generated for these data. SMOTE was applied in order to equal the number of minority class examples to the majority class, resulting in a base with 794 instances (50\%) of class 0 and 794 instances (50\%) of class 1 . The solution of the abovementioned three problems resulting thus in a database with 1588 (1000 real + 588 synthetic) patients and 596 columns (593 of (PR+RT) $+\mathrm{CD} 4+\mathrm{VL}+$ response to treatment). The use of the SMOTE technique improved our results considerably. 


\subsubsection{Classifiers considered}

Three different computational intelligence techniques were used for the design of the classifiers: MultiLayer Perceptron (MLP), Radial Basis Function (RBF), and Support Vector Machine (SVM).

For defining the best MLP configuration, we used multilayer perceptron networks with two intermediate layers, which are able to approximate any continuous function (CYBENKO, 1989). Different numbers of intermediate layers were considered, but none of them showed better results than two.

Because a very large number of connections can lead the network to memorize the training patterns instead of extracting their general characteristics and provide generalization, several values were tested for the number of nodes in the first intermediate layer(100, 150, 200, 250 , and 300), and for the number of nodes in the second intermediate layer $(50,75,100,125$, and 150). We also varied widely the rate of learning to observe the effect on the error, since a high learning rate makes the training very unstable and prevents the convergence of the learning process. The activation function chosen was hyperbolic tangent.

The learning algorithms tested were: Gradient descent with momentum and adaptive learning rate backpropagation (traingdx), since the momentum accelerates the training process and reduces the possibility of local minimum, whereas an adaptive learning rate attempts to keep the learning step size as large as possible, while keeping learning stable (MATLAB, 2011b); resilient backpropagation (trainrp), which is fast and uses little memory (MATLAB, 2011b), and one-step secant backpropagation (trainoss), which usually converges faster than conjugate gradient methods (ZAKARIA; ISA; SUANDI, 2010).

As stopping criterion, we used the maximum number of iterations equal to 1500 or max fail equal to 10, which indicates that if the error in the validation set grows for ten consecutive iterations, the training is stopped to avoid overfitting. The best configuration found for MLP had 250 nodes for the first intermediate layer, 125 nodes for the second intermediate layer, 0.001 for the learning rate and one-step secant backpropagation as learning algorithm.

The RBF has a fixed number of intermediate layers equal to one and number of neurons in the hidden layer is defined at run time, which gradually grows to reach the established mean 
square error taken as a parameter. Therefore, only one parameter was varied in search for the best possible value: the spread of radial basis functions that are used by each neuron. The values tested in this search were: from 1 (default) to 21 by a factor of 2 . These values were chosen because this parameter is directly associated with the smoothness of the approximation function, so that the higher the value, the smoother the function. Thus, a high spread means many neurons will be required to fit a fast-changing function while a low spread means a lot of neurons will be necessary to fit a smooth function and the network may not generalize well (MATLAB, 2011b). As stopping criterion we used a mean squared error goal equal to 0.05 . The RBF starts with no neuron in the hidden layer and, as the inputs are being provided, a new neuron is inserted with center equal to the input vector with the smallest error until it reaches the mean squared error specified as parameter (ABBIRAMY; TAMILARASI, 2011).

For SVM, according to Hsu, Chang and Lin (HSU; CHANG; LIN, 2000) there are three main parameters to be varied in search of the best classifier: kernel function, box constraint and rbf sigma. Kernel function is used by SVM to map the training data in the kernel space. Box constraint represents a restriction value for the soft margin and rbf sigma represents a scaling factor in the radial basis function kernel. In search for the best parameter settings, Hsu, Chang, and Lin (HSU; CHANG; LIN, 2000) advise starting with the kernel function "rbf" and default parameters. Then, in order to obtain a better accuracy, different box constraint values were tested (11 values, from $1 e^{-5}$ to $1 e^{5}$ by a factor of 10 .) and rbf sigma (11 values, from $1 e^{-5}$ to $1 e^{5}$ by a factor of 10).

For training the MLP, the data were divided into three sets: training, testing and validation. Worrying maintaining the proportions of the two classes in the three sets, the division was made as follows: we separated the the instances in accordance with the classes ( 0 and 1$)$; randomized both groups; each one was divided in ten equal parts, in order to realize a 10-fold cross validation; for each one of the ten runs of the training, one fold class 0 and one class 1 were put together at random to form the test set, while the other nine of each class were united randomly and divided into training set ( $2 / 3$ of the remaining instances) and validation ( $1 / 3$ of the remaining instances). This process was repeated 5 times to obtain a number of accuracy rates relevant to the statistical tests. For the training of RBF and SVM was performed similar process of dividing data. The 
difference is that they were only divided into two sets: training (80\%) and test (20\%).

For the experiments we used MATLAB R2011b (MATLAB, 2011b), which has a family of toolboxes with functions used to solve various types of problems and represent several processing structures. For the experiments with SVM, we used the Statistics Toolbox, and for MLP and RBF we used the Neural Network Toolbox.

\subsection{Results}

\subsubsection{Statistical Performance of the Methods}

The accuracy of the models created is presented in Table 7.1. According to the results, the SVM Model had the best performance, with $87.11 \%$ of correct predictions, while the RBF Model presented $84.34 \%$, and the MLP Model, $71.02 \%$. The distance between MLP and SVM is about 16 percentage points, and between SVM and RBF is 2.77 percentage points. Based on this observation, we can conclude that the MLP Model had the worst performance, because the difference of the MLP results to the other methods was highly significant. SVM and RBF had close percentages of correct predictions, for checking if there is statistical difference between them, an appropriate test was performed, as described next.

To verify the normality of the data and thus identify the most appropriate statistical test to find out if there is statistical evidence that SVM was better than RBF, two Kolmogorov-Smirnov tests (MONTGOMERY; RUNGER, 2003) were applied, one for each model. For RBF we have $H_{0}: X \sim N\left(\mu=84.33613 ; \sigma^{2}=6.227\right)$ and $H_{1}: X \sim$ other distribution. For SVM we have $H_{0}: X \sim N\left(\mu=87.11225 ; \sigma^{2}=6.7943\right)$ and $H_{1}: X \sim$ other distribution. The values of the test statistic obtained for RBF and SVM were $D=0.1367$ and $D=0.1251$, respectively. As the critical value for a sample of size 50 and a significance level of 0.05 is equal to 0.1923 , we do not reject the hypothesis that the data come from a normal distribution.

Given that the data follow a normal distribution, a t-test was performed to check for statistical evidence that the SVM showed better results than the RBF. For $H_{0}: \mu_{S V M}=\mu_{R B F}$ and $H_{1}: \mu_{S V M}>\mu_{R B F}$, with confidence level of $95 \%$, the test statistic was 5.3853 and p-value 2.489e-07. Thus, as the rejection region for the null hypothesis, considering 98 degrees of 
freedom, is $\mathrm{t}>1.658$, we reject the null hypothesis with $\alpha=0.05$ and we conclude that the SVM has accuracy rates higher than those obtained by the RBF.

Another evidence of the superiority of SVM can be seen in the ROC curves. Figure 7.2 illustrates the ROC curves for the three classifiers, considering all patients of 50 folds. The MLP had an area under the curve of 0.7824 , while the RBF had a larger area of 0.925 , very close, but still smaller than the area obtained by SVM, 0.9398.

The accuracy of models for each class is presented in Table 7.1. According to the results, the SVM and the MLP models showed no significant differences in performance, indicating that the synthetic patients created by the SMOTE were able to extend the decision region of the minority class without increasing the bias of the classifier for that class, and thus increased the generalization power of classifiers. The RBF model was slightly more sensitive to synthetic data. In this model, the minority class showed a mean accuracy 11.55 percentage points higher than the other class, indicating a greater bias towards the minority class than other models, but not considerably affecting the generalization of the network, since it presented high accuracy.

Table 7.1: Performance of Models for each class: $(\mu)$ mean percentage of correct predictions; and $(\sigma)$ standard deviation.

\begin{tabular}{|c|c|c|c|}
\hline Model & Class & $\mu$ & $\sigma$ \\
\hline \multirow{3}{*}{ MLP } & 0 & $71.25 \%$ & 6.72 \\
\cline { 2 - 4 } & 1 & $70.80 \%$ & 10.66 \\
\cline { 2 - 4 } & Total & $71.02 \%$ & 5.38 \\
\hline \multirow{2}{*}{ RBF } & 0 & $78.56 \%$ & 3.42 \\
\cline { 2 - 4 } & 1 & $90.11 \%$ & 3.70 \\
\cline { 2 - 4 } & Total & $84.34 \%$ & 2.49 \\
\hline \multirow{2}{*}{ SVM } & 0 & $85.71 \%$ & 3.33 \\
\cline { 2 - 4 } & 1 & $88.51 \%$ & 3.72 \\
\cline { 2 - 4 } & Total & $87.11 \%$ & 2.61 \\
\hline
\end{tabular}

\subsubsection{Qualitative analysis of results}

For each patient, a consensus response was computed for each model, using a simple metric: The majority response of 5 predictions. Figure 7.3 presents a Venn Diagram of consensus responses predicted incorrectly. From a universe of 1000 patients, 646 patients (called the EASY 


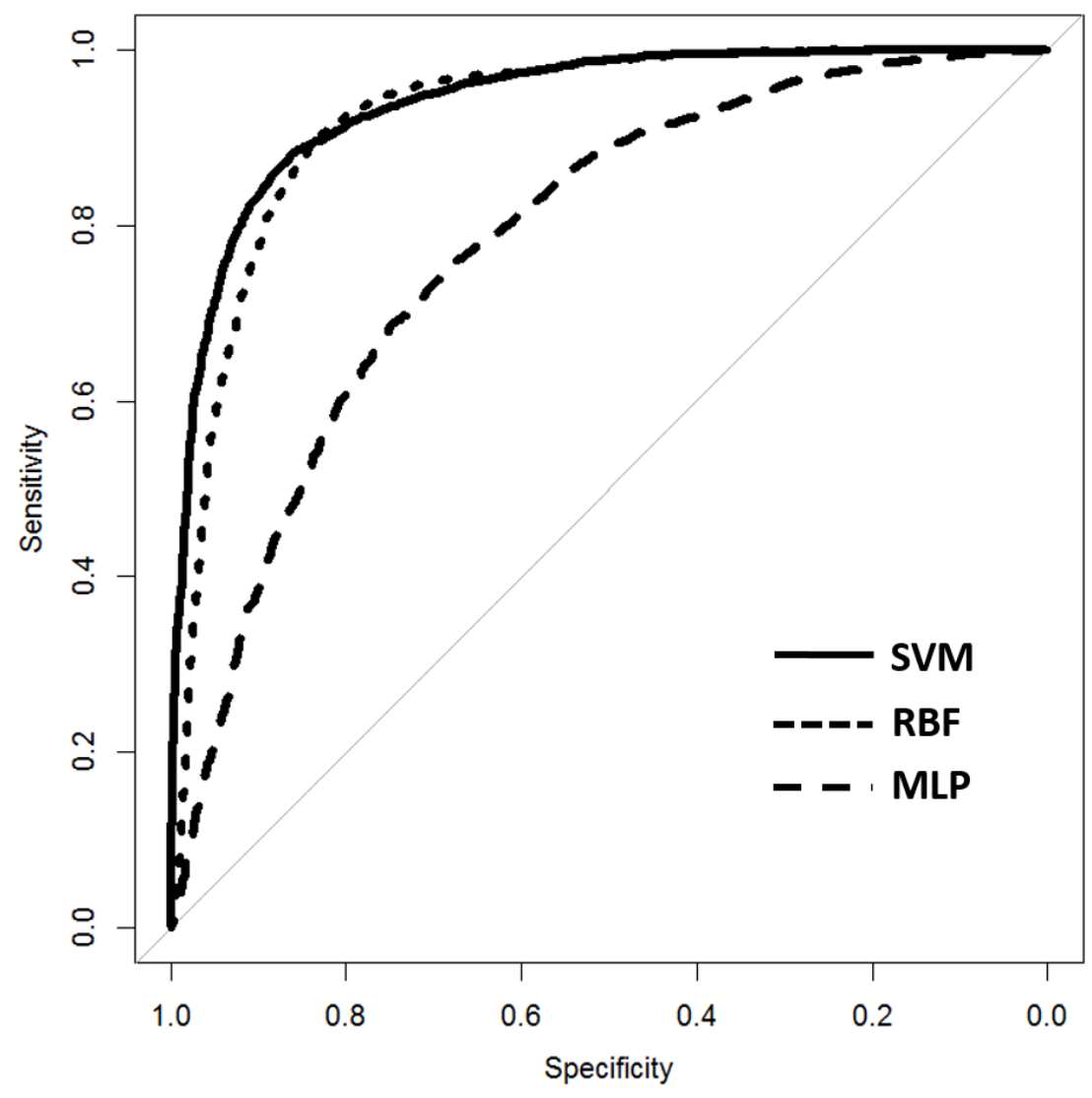

Figure 7.2: ROC curves considering results of 50 runs of three Models.

group) had responses correctly predicted by all models, while for 69 patients (called the HARD group) all models failed in finding the correct response. With 19 exclusive (not shared with any other model) incorrect predictions, SVM was the most accurate model. The model with the largest number of predictions, both exclusive and in general, was MLP (99).

For the remainder of this Section, we focus our attention on identifying which properties in the data would separate the 69 (incorrectly predicted by all three methods) patients in the HARD group from the 646 (correctly predicted by all three methods) individuals in the EASY group.

Initially, the VL and CD4+ count were analyzed. Table 7.2 presents the average of VL and CD4+ in these two groups. The average VL was very close in the two groups (4.19 in the EASY and 4.86 in the HARD group). We did not find evidences that there is correlation between incorrect inferences and VL. On the other hand, CD4+ count presented significant difference between the two groups. While in the EASY group the CD4+ count average was 293.39, in the 


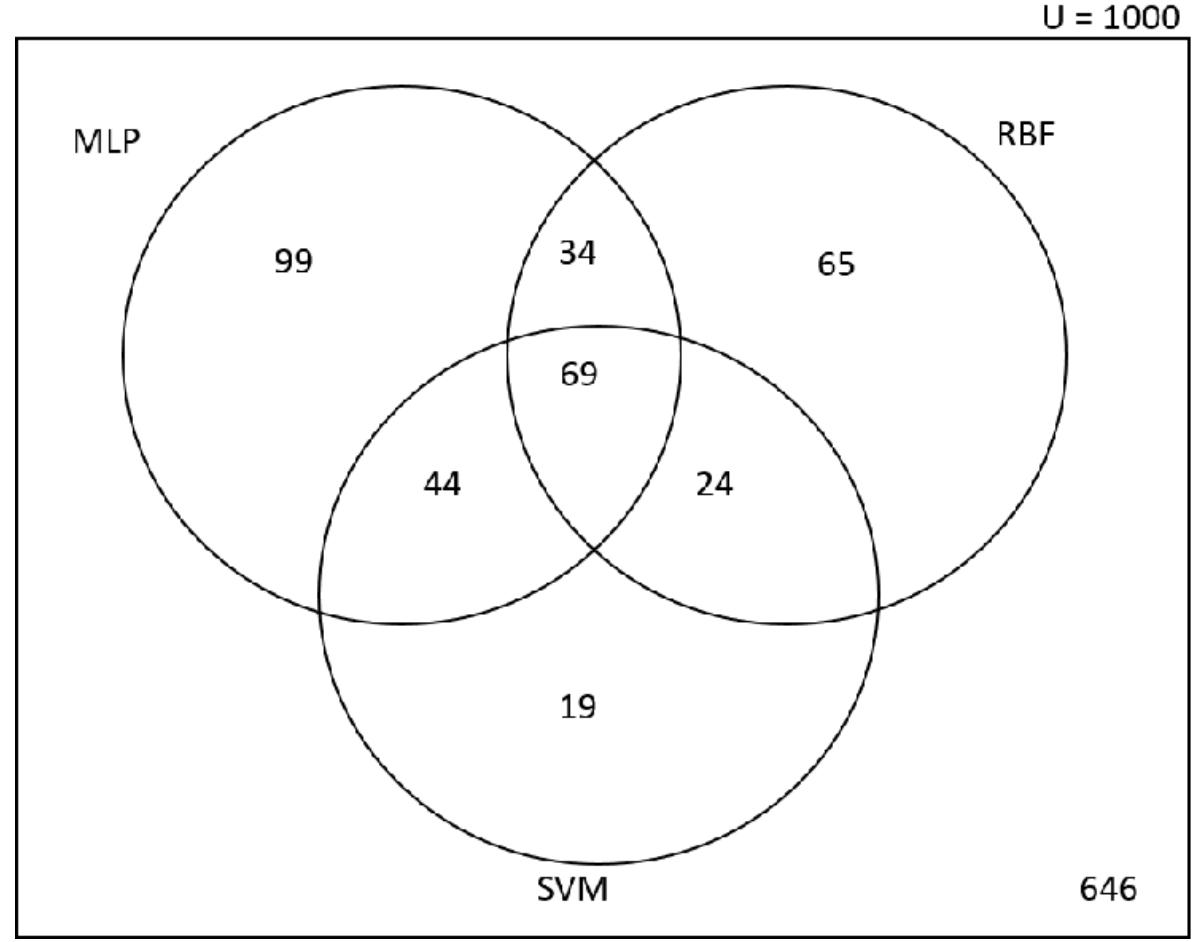

Figure 7.3: Venn Diagram of patient's consensus response predicted incorrectly. The response for each patient was considered the consensus class in 5 predictions in each model.

HARD group it was 184.88 .

Table 7.2: The average(standard deviation) of Viral Load and CD4+ for EASY and HARD groups.

\begin{tabular}{|c|c|c|}
\hline Property & EASY group & HARD group \\
\hline Viral Load & $4.19(0.7)$ & $4.86(0.5)$ \\
\hline CD4+ & $293.39(197.08)$ & $184.88(156.12)$ \\
\hline
\end{tabular}

We also investigated the distribution of classes in each group. Table 7.3 shows the percentage of responders and non-responders in each group. There is significant difference between the frequency of each class in each group (9.49 percentage points). However, we did not find a linear correlation between CD4+ and occurrence of errors in predictions. We fitted decision trees for identifying the frequency of responders/non-responders according to VL and CD4+. Figure 7.4 shows the decision tree to CD4+ count, the frequency of patients responders with CD4+ count $\leq 151$ is $70.4 \%$ while in patients with count $>151$ is $83.3 \%$. A similar behavior of distribution of responders and non-responders is observed in VL. Figure 7.5 shows that patients 
with $\mathrm{VL} \leq 3.85$ had more difficult for responding to treatment (96.9\% non-responders and $3.1 \%$ responders). The concentration of responders grows with VL, when VL $>4.65$ the frequency of responders is $38.9 \%$.

Table 7.3: The distribution of the classes in EASY and HARD group.

\begin{tabular}{|c|c|c|}
\hline Class & non-responders & responders \\
\hline EASY group & $80.5 \%$ & $19.5 \%$ \\
\hline HARD group & $71.01 \%$ & $28.99 \%$ \\
\hline
\end{tabular}

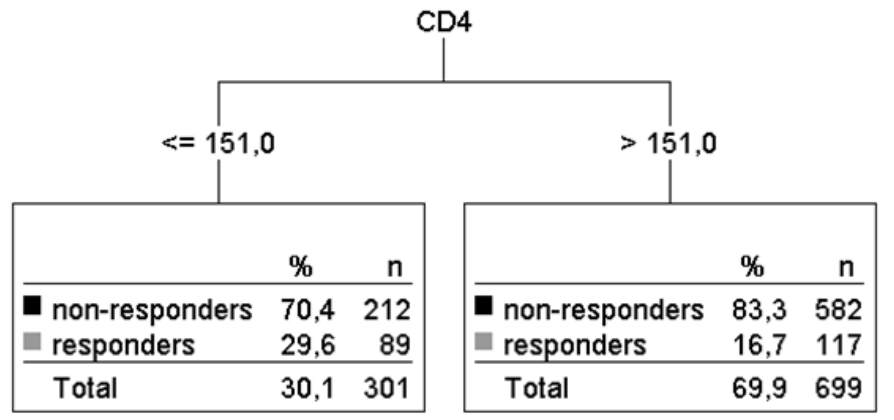

Figure 7.4: Decision Tree fitted considering CD4+ count at start of treatment.

Additional information is required for investigating which properties would be able to characterize each patient's group individually. We used decision trees for identifying differences among the RT and PR amino acid sequences in each group.

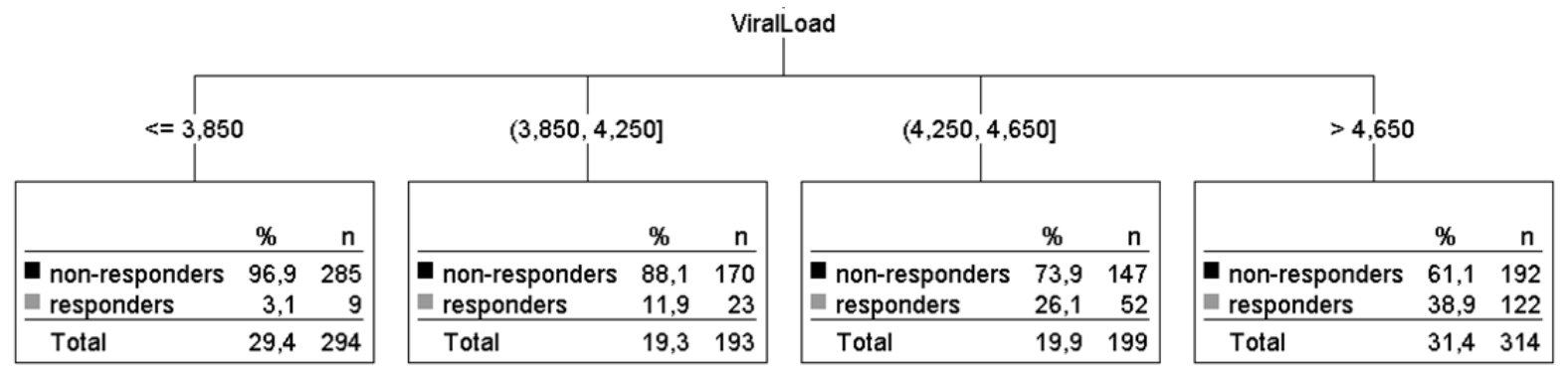

Figure 7.5: Decision Tree fitted considering Viral Load at start of treatment.

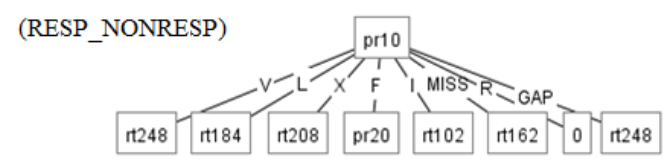

(EASY_HARD)

Figure 7.6: Decision Trees estimated considering all codons of RT and PR for:

(RESP_NONRESP) 1000 patients and their actual classes: responders and non-responders; and (EASY_HARD) EASY and HARD group. 

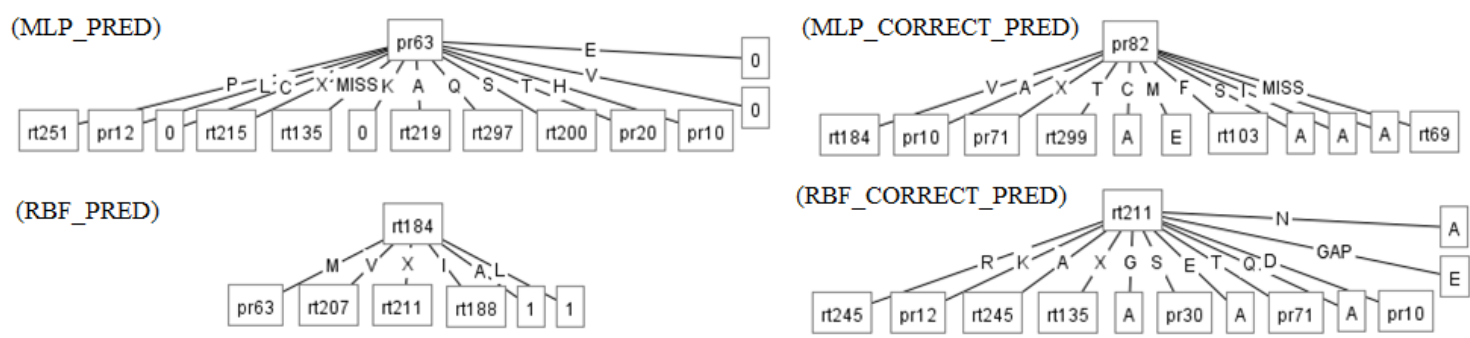

(SVM PRED)

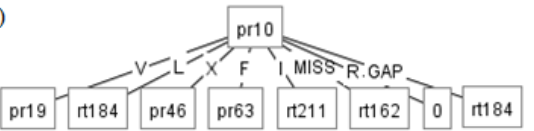

(SVM_CORRECT_PRED)

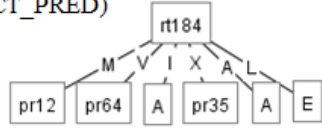

Figure 7.7: Decision Trees estimated considering all codons of RT and PR for: predicted classes of all models (MLP_PRED, RBF_PRED and SVM_PRED); and correct and incorrect predictions in each model (MLP_CORRECT_PRED, RBF_CORRECT_PRED and SVM_CORRECT_PRED).

Figure 7.6 presents two models: (RESP_NONRESP) the raw data, 1000 patients separated by actual class, where 1 denotes responders and 0 denotes non-responders, and (EASY_HARD) Patients from groups EASY (646 samples) and HARD (69 samples). Figure 7.7 presents six models: MLP_PRED, RBF_PRED and SVM_PRED were estimated considering the predicted classes by each model, and MLP_CORRECT_PRED, RBF_CORRECT_PRED and SVM_CORRECT_PRED the outputs of each model are classified in correct or incorrect prediction.

Figure 7.6 (RESP_NONRESP) presents the model where we are looking for the codons that are more relevant for the classification task. According to the tree built, pr10 is the most significant codon for separating responsers from non-responders in general. We did not observe any difference in the frequencies of amino acids on this codon.

The tree Figure 7.6 (EASY_HARD) models the codon which best separates patients correctly and incorrectly predicted. rt184 was the most important codon for it. The group of patients predicted incorrectly presented two possible amino acids translated in codon rt184: Methionine (M), and Valine (V). The relative frequencies of these amino acids in this group were: (M) $76.82 \%$ and (V) $23.18 \%$. The following amino acids occur in this codon in the EASY group: (I) Isoleucine 1.4\%, Methionine 39.16\% (M), Valine 55.88\%(V), and a non standard amino acid $3.56 \%(\mathrm{X})$. Codons rt211 and rt296 were indicated as two of the most important codons. The literature contains literature references to codon rt211 (MARCELIN et al., 2006), but we could not find references to codon rt296. 
The trees in Figure 7.7 (MLP_PRED-MLP_CORRECT_PRED) were fitted for comparing if there are differences on the prediction power of codons, considering predicted classes and correct and incorrect predictions in the MLP model(MLP_PRED-MLP_CORRECT_PRED, respectively). When the tree to consider predicted classes the most important codon is pr63. The most important codon for separating correct and incorrect predictions in the MLP model is pr82. Considering codon pr82 we observed two significant differences between frequencies of amino acids in correct and incorrect predictions. In the EASY group predictions: Alanine $16.16 \%$ (A) and missing codons $3.84 \%$. In the HARD group predictions: Alanine $3.27 \%$ (A) and missing codons $20.82 \%$. So, there is, indeed, considerable differences regarding this codon in the two scenarios.

The trees in Figure 7.7 (RBF_PRED-RBF_CORRECT_PRED) were fitted for the RBF model. In the tree that considers predicted classes, the most important codon is rt184. The most important codon for separating correct and incorrect predictions in the RBF model is pr211. Considering codon rt184 we observed two significant differences between frequencies of amino acids in responders and non-responders. In responders: Methionine 59.14\% (M) and Valine $36.88 \%$ (V). In non-responders: Methionine $41.49 \%(\mathrm{M})$ and Valine $53.36 \%$ (V).

SVM is the method that presented the best performance, its results are analyzed in Figure 7.7 (SVM_PRED-SVM_CORRECT_PRED). The most important codon for separating the patients considering the class predicted by SVM is pr10, and for separating its correct and incorrect predictions is rt184. Considering codon rt184 we observed two significant differences between frequencies of amino acids in correct and incorrect predictions. In correct: Methionine 42.30\% (M) and Valine 52.37\% (V). In incorrect: Methionine 71.15\% (M) and Valine 26.92\% $(\mathrm{V})$.

The differences detected in the frequencies of the identified amino acids are a strong evidence to validate the binary tree models developed.

\subsection{Discussion and Conclusions}

We did an extensive study of different types of genomic data taken from the HIV Resistance Drug Database and its relation to the prediction of patient's response to anti-HIV 
treatment.

Due to the lack of balance between the case and control samples available, the use of SMOTE technique was central to the success of our analysis. The SMOTE algorithm was applied for resolving the unbalance problem of the data, creating synthetic patients rather than simply replicating a portion of the smaller class. The analysis was based on three well known computational intelligence methods: MLP, RBF, and SVM. We used statistical tests and ROC analysis for comparison of the accuracy of the methods. Decision trees helped the identification or confirm relationships between: (1) RT/PR codons and predicted classes, (2) RT/PR codons and correct and incorrect predictions, and (3) CD4+/VL and responder and non-responder patients. The results of our analysis offer valuable insights on the behavior of those methods.

We found that the RBF and the SVM models have similar accuracy, although statistically SVM was the best, both being more accurate than MLP by a large margin. Interestingly, RBF is more sensitive than SVM to synthetic samples created by the SMOTE algorithm. While predicted classes by SVM were proportional to frequency of actual classes, RBF was not.

A Venn Diagram was built for analyzing the number of patients' responses predicted incorrectly that are shared by the methods. We identified 646 samples predicted correctly by all methods in all tests, and 69 samples predicted incorrectly in the same situation. SVM had 19 incorrect exclusive predictions, while RBF had 65, and MLP had 99. Based on this observation we can conclude that SVM tends to fail only when the others methods also fail.

We fitted a decision tree for recognizing patterns that separate 69 patients predicted incorrectly from 646 patients correctly predicted. Codon rt184 was identified as the best property for characterizing these two patient groups. Several works have related mutations on this codon to resistance to therapy anti-HIV (KULKARNI et al., 2011; ANTA et al., 2012; XU et al., 2013).

Indeed, three decision trees had as root codon rt184 (Figures 7.6, (RESP_NONRESP), and 7.7 (RBF_PRED-SVM_CORRECT_PRED)). We computed the relative frequency of amino acids on this codon for each class considered by the trees. Significant differences of frequency of amino acids between the classes of the trees Figure 7.6 (EASY_HARD) and Figure 7.7 (SVM_CORRECT_PRED) were found, considering as classes correct and incorrect predictions. Tree Figure 7.6 (EASY_HARD) was fitted with 69 patients predicted incorrectly from 646 
Table 7.4: Relative Frequency of Patients by Class, CD4+ Count and Viral Load.

\begin{tabular}{|c|c|c|r|r|}
\hline CD4+ data & \# Patients & Class $(\%)$ & $\mathrm{CV} \mu(\sigma)$ & $\mathrm{CD} 4+\mu(\sigma)$ \\
\hline \multirow{2}{*}{ All } & \multirow{2}{*}{$1000(100 \%)$} & $0(79.4)$ & $4.18(0.68)$ & $291.60(193.65)$ \\
\cline { 3 - 5 } & & $1(20.6)$ & $4.80(0.56)$ & $233.50(207.96)$ \\
\hline \multirow{2}{*}{$\leq 350$} & \multirow{2}{*}{$701(70.1 \%)$} & $0(76.6)$ & $4.32(0.69)$ & $183.11(095.33)$ \\
\cline { 3 - 5 } & & $1(23.4)$ & $4.85(0.54)$ & $155.11(105.51)$ \\
\hline \multirow{2}{*}{$>350$} & \multirow{2}{*}{$299(29.9 \%))$} & $0(86.3)$ & $3.85(0.52)$ & $516.36(141.40)$ \\
\cline { 3 - 5 } & & $1(14.7)$ & $4.62(0.60)$ & $548.71(243.69)$ \\
\hline
\end{tabular}

correct for all methods, and tree Figure 7.7 (SVM_CORRECT_PRED) considers the correct and incorrect prediction of the SVM model only. An inverse correlation was observed in frequency of Methionine (M) and Valine (V) between classes in both trees. In the tree Figure 7.6 (EASY_HARD): EASY group ( $\mathrm{M}=39.16 \%$ and 55.88\%); and HARD group $(\mathrm{M}=76.82 \%$ and V=23.18\%). In tree Figure 7.7 (SVM_CORRECT_PRED): EASY group (M=42.30\% and $\mathrm{V}=52.37 \%)$; and HARD group ( $\mathrm{M}=42.30 \%$ and $52.37 \%)$. Methionine was more abundant in the patients inferred incorrectly. Methionine at rt184 is found in wild-type HIV, which is later replaced by Valine (FROST et al., 2000).

Tree Figure 7.6 (EASY_HARD) has rt184 as principal property to separate correct from incorrect predictions. Tree Figure 7.6 (RESP_NONRESP) has codon pr10, largely mentioned in the literature (ALTERI et al., 2013; HAIDARA et al., 2012), as the main observed mutation which leads to failure of the treatment of the patients studied. Model SVM had the best performance in the test done, and, curiously, codon rt184 was also identified as the one which may induce SVM to go wrong (Figure 7.7 (SVM_CORRECT_PRED)). For model SVM, codon pr10 had the most predictive power (Figure 7.7 (SVM_PRED)), as well as in the tree constructed considering the actual classes (Figure 7.6 (RESP_NONRESP)). For all that was presented here, we can conclude that codon rt184 needs special treatment in the models, since, although it is associated to the anti-HIV drug resistance, it may also induce the preditors to errors.

Table 7.4 presents the distribution of patients by class. From the patients that started treatment for having $(\mathrm{CD} 4+>350), 86.3 \%$ did not present a good response to therapy, while $13.7 \%$ responded satisfactorily (with considerable reduction in their viral load). That could be explained by a number of reasons. Such patients have average viral load of 3.85, versus 4.62, for 
the others. Since the immunologic system of those patients were normal and with low viral load, the reason to start the anti-HIV treatment was not to strike out the virus directly, but to prevent an injury of the immune system, or an infection from mother to son, for instance. Since they are healthy, the $\mathrm{CD}+$ and VL values remain unchanged, and they are considered as non-responders.

The goal of the anti-HIV therapy is to reduce the viral load, thus decreasing the aggression to the immune system, enabling it to recover and increase the amount of CD4+ cells. When the viral load is very high it causes damages that are difficult to repair. A very low CD4+ value is evidence that the immune system is highly beaten. Thus, it would be logical to think that the higher the VL value, the greater the chances of the patient not responding to the treatment, as well as patients with low CD4+ measures. But our analysis of the data points in another direction (Figures 7.4 and 7.5).

The response to the virus therapy is immediate, since the virus lasts about 48 hours, and the decrease in the VL is used to determine whether the patient had or not a good response to the treatment. On the other hand, the immune response (increased CD4+) is slower. A patient with low CD4+ may have had a 10-fold reduction of VL at 16 weeks, but his CD4+ may never recover due to the high aggression that the immune system may have suffered. This scenario is not analyzed in our study, since the available data are old, from a time that survival was limited, so there is no information regarding for a long period of patient follow up.

Figure 7.4 shows that the lower the CD4+ (values less than or equal to 151), the greater the chance of viral load reduction (responders); the average VL for responders with CD4+ $\leq 151$ was 5.029, while for patients with CD4+ > 151 the average reduction was 4.617.

Figure 7.5 tells us that the higher the viral load, the higher chances of it being reduced. Responders with CD4+ $\leq 151$ had higher viral loads than those with higher CD4+.

We hypothesize that the greater the amount of virus, the greater the chances of them being reduced at 16 weeks, regardless of the state of the patient's immune system, however, this reduction means just a chance for the immune system to recover, which may or may not happen. We note that in our data there is a considerable proportion of deaths among patients who entered late in therapy (very low CD4+). For example, from 2003 to 2006, Brazil recorded a total of 50.358 patients late in their health system. Of those, 14,457 (28.7\%) died within 20 days, and 
3,299 (6.55\%) had symptoms of developing AIDS (GRANGEIRO et al., 2011).

We believe that the insights provided by our analysis can be used for a more effective choice of models, and can also be explored in the design of better approaches for prediction of patients' response to anti-HIV therapies. 


\section{8}

\section{Conclusions}

This thesis contains an extensive analysis of errors in Haplotype Inference methods. We verified that HI methods tend to fail in different locations of DNA sequences. This insight motivated us to construct models for predicting errors of HI by different algorithms. Neural Network (NN) and Multiple Linear Regression (MLR) models were proposed, with the latter showing the best results. The MLR models were very accurate for predicting Switch Error (SE), in most tests performed the median of the relative error was close to $1 \%$. The results show us that inferring HI errors by individuals is more promising than by SNPs. We also proposed a new method, called HybHap, based on Markov chains, which were central to the performance of models on Individuals base.

Although the models developed for the SNPs base had a weak performance, the analysis of the impact of Linkage Disequilibrium developed on this base produced several interesting novel insights about the HI problem: (1) The upstream region had a stronger impact in the occurrence of errors in HI algorithms than the downstream region; (2) Considering a window of 10 SNPs in the upstream region makes the LD estimation more precise than with smaller width; (3) Eliminating non-informative SNPs identified by the Exact Fisher's Test also improves the precision of LD estimation; (4) Regions with low and high LD levels are easier for predicting errors than others; (5) Regions with mid-low LD level do not present evidence of correlation between LD and occurrence of errors in HI algorithms, which means that for SNPs located in those regions, the LD is non-informative for Haplotype Inference. 
Based on evidences that several properties analysed are good regressors for predicting Switch Error on Individuals, we developed a novel ensemble method for Haplotype Inference solutions considering internal $\mathrm{HI}$ algorithm variability. The strategy is to consider as the most reliable solution the haplotype pair that is the most similar to the others, based on the minimization of Switch Error on Individuals across several candidate solutions. This approach is already developed, but a theoretical proof of correctness and complexity is still being developed.

Finally, the framework used in our analyses was applied for investigating the connection between the response of patients to anti-HIV treatment and properties of RNA sequences of the virus. In this practical investigation we considered sequences of reverse transcriptase and protease. Among other things, the results showed evidences that codon pr10 of protease is the most important property for identifying patients with a positive response to the treatment, and the machine learning methods tends to fail when the viral strain is wild.

Different parts of this thesis has been published as scientific articles in several conferences in the last few years. We plan to work further on the haplotype inference problem, particularly concluding the proof of correctness of the Switch Error predictor developed. 


\section{References}

ABBIRAMY, V. S.; TAMILARASI, A. A Comparative Study on Human Spermatozoa Images Classification with Artificial Neural Network Based on FOS, GLCM and Morphological Features. In: ADVANCES IN DIGITAL IMAGE PROCESSING AND INFORMATION TECHNOLOGY. Anais... [S.1.: s.n.], 2011. p.220-228.

ADKINS, R. M. Comparison of the accuracy of methods of computational haplotype inference using a large empirical dataset. BMC Genetics, [S.1.], v.5, n.22, 2004.

ALTERI, C. et al. Structural modifications induced by specific HIV-1 protease-compensatory mutations have an impact on the virological response to a first-line lopinavir/ritonavir-containing regimen. Journal of Antimicrobial Chemotherapy, [S.1.], 2013.

ALTMANN, A. et al. Predicting the response to combination antiretroviral therapy: retrospective validation of geno2pheno-THEO on a large clinical database. Journal of Infectious Diseases, [S.1.], v.199, n.7, p.999-1006, 2009.

ANTA, L. et al. Rilpivirine Resistance Mutations in HIV Patients Failing Non-Nucleoside Reverse Transcriptase Inhibitor-Based Therapies. Journal of Acquired Immune Deficiency Syndromes, [S.1.], 2012.

BROWN, D. G.; HARROWER, I. M. Integer Programming Approaches to Haplotype Inference by Pure Parsimony. IEEE/ACM Transactions on Computational Biology and

Bioinformatics, [S.1.], v.3, n.2, p.141-154, 2006.

BROWNING, B. L.; BROWNING, S. R. A Unified Approach to Genotype Imputation and Haplotype-Phase Inference for Large Data Sets of Trios and Unrelated Individuals. American journal of human genetics, [S.1.], v.84, n.2, p.210-223, 2009.

BROWNING, B. L.; BROWNING, S. R. A fast, powerful method for detecting identity by descent. American journal of human genetics, [S.1.], v.88, n.2, p.173-82, 2011.

CHAWLA, N. V. et al. SMOTE: synthetic minority over-sampling technique. Journal of Artificial Intelligence Research, [S.1.], v.16, n.1, p.321-357, 2002.

CHE, D.; TANG, H.; SONG, Y. Haplotype inference using a genetic algorithm. In: CIBCB. Anais... IEEE, 2009. p.31-37.

CHING, A. et al. SNP frequency, haplotype structure and linkage disequilibrium in elite maize inbred lines. BMC Genetics, [S.1.], v.3, n.1, p.19+, 2002.

CLARK, A. Inference of haplotypes from PCR amplified samples of diploid populations. Journal of Molecular Biology and Evolution, [S.1.], v.7, p.111-122, 1990.

CYBENKO, G. Approximation by superpositions of a Sigmoid function. Mathematics of Control, Signals, and Systems, [S.1.], v.2, p.303-314, 1989.

DING, Z.; FILKOV, V.; GUSFIELD, D. A linear-time algorithm for the perfect phylogeny haplotyping (PPH) problem. In: INTERNATIONAL CONFERENCE ON RESEARCH IN COMPUTATIONAL MOLECULAR BIOLOGY (RECOMB). Anais... [S.l.: s.n.], 2005. p.585-600. 
ERONEN, L.; GEERTS, F.; TOIVONEN, H. A Markov Chain Approach to Reconstruction of Long Haplotypes. In: PACIFIC SYMPOSIUM ON BIOCOMPUTING. Anais... World Scientific, 2004. p.104-115.

ERONEN, L.; GEERTS, F.; TOIVONEN, H. HaploRec: efficient and accurate large-scale reconstruction of haplotypes. BMC Bioinformatics, [S.1.], v.7, p.542, 2006.

FROST, S. D. et al. Evolution of lamivudine resistance in human immunodeficiency virus type 1-infected individuals: the relative roles of drift and selection. Journal of Virology, [S.1.], v.74, n.14, p.6262-8, 2000.

GOUJON, M. et al. A new bioinformatics analysis tools framework at EMBL-EBI. Nucleic Acids Research, [S.1.], v.38, n.suppl 2, p.W695-W699, July 2010.

GRANGEIRO, A. et al. Late Entry into HIV Care: estimated impact on aids mortality rates in brazil, 2003-2006. PLOS One, [S.1.], v.6, 2011.

GUSFIELD, D. Inference of Haplotypes from samples of diploids populations: complexity and algorithms. Journal of Computational Biology, [S.1.], v.8, p.305-323, 2001.

GUSFIELD, D. Haplotyping as Perfect Phylogeny: conceptual framework and efficient solutions. In: INTERNATIONAL CONFERENCE ON RESEARCH IN COMPUTATIONAL MOLECULAR BIOLOGY (RECOMB). Anais... [S.1.: s.n.], 2002. p.166-175.

GUSFIELD, D. Haplotype Inference by Pure Parsimony. In: COMBINATORIAL PATTERN MATCHING. Anais... [S.1.: s.n.], 2003. v.2676, p.144-155.

HAIDARA, A. et al. Drug Resistance Pathways and Impact of Protease Mutation L10I/V in HIV-1 Non-B Subtypes. Journal of Antivirals and Antiretrovirals, [S.1.], v.4, n.2, p.40, 2012.

HALLDóRSSON, B. V. et al. A survey of computational methods for determining haplotypes. In: LECTURE NOTES IN COMPUTER SCIENCE: COMPUTATIONAL METHODS FOR SNPS AND HAPLOTYPE INFERENCE. Anais... [S.1.: s.n.], 2004. v.2983, p.26-47.

HEIDER, D. et al. Multilabel classification for exploiting cross-resistance information in HIV-1 drug resistance prediction. Bioinformatics, [S.1.], 2013.

HIGASA, K. et al. Evaluation of Haplotype Inference Using Definitive Haplotype Data Obtained from Complete Hydatidiform Moles, and Its Significance for the Analyses of Positively Selected Regions. PLoS Genetics, [S.1.], v.5, n.5, 2009.

HSU, C.-W.; CHANG, C.-C.; LIN, C.-J. A Practical Guide to Support Vector Classification. 2000 .

KULKARNI, R. et al. The HIV-1 Reverse Transcriptase M184I Mutation Enhances the E138K-associated Resistance to Rilpivirine and Decreases Viral Fitness. Journal of Acquired Immune Deficiency Syndromes, [S.1.], 2011.

LANCIA, G.; PINOTTI, C. M.; RIZZI, R. Haplotype Haplotyping Populations by Pure Parsimony: complexity of exact and approximation algorithms. INFORMS J. Computing, [S.1.], v.16, p.348-359, 2004.

LARDER, B. et al. The development of artificial neural networks to predict virological response to combination HIV therapy. Antiviral therapy, [S.1.], v.12, n.1, p.15-24, 2007. 
LI, Z. et al. A parsimonious tree-grow method for haplotype inference. Bioinformatics, [S.1.], v.21, p.3475-3481, 2005.

LIN, S. et al. Haplotype inference in random population samples. American Journal of Human Genetics, [S.1.], v.71, n.5, p.1129-1137, 2002.

MANSKY, L. M. Retrovirus mutation rates and their role in genetic variation. Journal of General Virology, [S.1.], n.79, p.1337-1345, 1998.

MARCELIN, A.-G. et al. Impact of HIV-1 reverse transcriptase polymorphism at codons 211 and 228 on virological response to didanosine. Antiviral Therapy, [S.1.], v.11, n.6, p.693-9, 2006.

MATLAB. version 7.13.0.564 (R2011b). Natick, Massachusetts: The MathWorks Inc., 2011.

MATLAB. [S.1.]: The MathWorks Inc, 2011.

MONTGOMERY, D.; RUNGER, G. Applied statistics and probability for engineers. 4.ed. [S.1.]: LTC, 2003.

NIU, T. et al. Bayesian Haplotype Inference for Multiple Linked Single-Nucleotide Polymorphisms. American Journal of Human Genetics, [S.1.], v.70, p.157-169, 2002.

PROSPERI, M. C. F. et al. Antiretroviral therapy optimisation without genotype resistance testing: a perspective on treatment history based models. PLoS One, [S.1.], v.5, n.10, p.e13753, 2010 .

R Development Core Team. R: a language and environment for statistical computing. Vienna, Austria: R Foundation for Statistical Computing, 2011.

REVELL, A. D. et al. Modelling response to HIV therapy without a genotype: an argument for viral load monitoring in resource-limited settings. Journal of Antimicrobial Chemotherapy, [S.1.], v.65, n.4, p.605-7, 2010.

REVELL, A. D. et al. Computational models can predict response to HIV therapy without a genotype and may reduce treatment failure in different resource-limited settings. Journal of Antimicrobial Chemotherapy, [S.1.], 2013.

RHEE, S.-Y. et al. Human immunodeficiency virus reverse transcriptase and protease sequence database. Nucleic Acids Research, [S.1.], v.31, n.1, p.298-303, 2003.

RICE, P.; LONGDEN, I.; BLEASBY, A. EMBOSS: the european molecular biology open software suite. Trends Genet, [S.1.], v.16, n.6, p.276-7, 2000.

ROSA, R. S. et al. Insights on prediction of patients' response to anti-HIV therapies through machine learning. In: INTERNATIONAL JOINT CONFERENCE ON NEURAL NETWORKS, IJCNN 2014, BEIJING, CHINA, JULY 6-11, 2014, 2014. Anais... [S.1.: s.n.], 2014. p.3697-3704.

ROSA, R. S.; GUIMARÃES, K. S. HybHap: a fast and accurate hybrid approach for haplotype inference on large datasets. In: BSB. Anais... Springer, 2013. p.24-35. (Lecture Notes in Computer Science, v.8213). 
ROSA, R. S.; GUIMARÃES, K. S. Insights on Haplotype Inference on Large Genotype Datasets. In: LECTURE NOTES ON COMPUTES SCIENCE. Anais... [S.1.: s.n.], 2010. v.6268, p.47-58.

ROSA, R. S.; SANTOS, R. H. S.; GUIMARÃES, K. S. Accurate Prediction of Error in Haplotype Inference Methods through Neural Networks. In: IEEE IJCNN 2012. Proceedings... [S.1.: s.n.], 2012. p.1-8.

ROSA, R. S.; SANTOS, R. H. S.; GUIMARÃES, K. S. Associating Genotype Sequence Properties to Haplotype Inference Errors. In: ADVANCES IN BIOINFORMATICS AND COMPUTATIONAL BIOLOGY - 7TH BRAZILIAN SYMPOSIUM ON BIOINFORMATICS, BSB 2012, CAMPO GRANDE, BRAZIL, AUGUST 15-17, 2012. PROCEEDINGS. Anais... [S.1.: s.n.], 2012. p.132-143.

SCHEET, P.; STEPHENS, M. A fast and flexible statistical model for large-scale population genotype data: applications to inferring missing genotypes and haplotypic phase. American Journal of Human Genetics, [S.1.], v.78, n.4, p.629-44, 2006.

SIEVERS, F. et al. Fast, scalable generation of high-quality protein multiple sequence alignments using Clustal Omega. Molecular Systems Biology, [S.1.], v.7, 2011.

SMITH-MILES, K. A. Cross-disciplinary Perspectives on Meta-learning for Algorithm Selection. ACM Computing Surveys, New York, NY, USA, v.41, n.1, p.6:1-6:25, Jan. 2009.

STEPHENS, M.; DONNELLY, P. A Comparison of Bayesian methods for haplotype reconstruction from population genotype data. American Journal of Human Genetics, [S.1.], v.73, p.1162-1169, 2003.

STEPHENS, M.; SMITH, N.; DONNELLY, P. A new statistical method for haplotype reconstruction from population data. American Journal of Human Genetics, [S.1.], v.68, p.978-989, 2001.

SUN, S.; GREENWOOD, C. M.; NEAL, R. M. Haplotype inference using a Bayesian Hidden Markov model. genetic epidemiology, [S.1.], v.31, p.937-948, 2007.

The International HapMap Consortium. The International HapMap Consortium. Nature, [S.1.], v.426, p.789-796, 2003.

TINININI, L. et al. CollHaps: a heuristic approach to haplotype inference by parsimony. IEEE/ACM Transactions on Computational Biology and Bioinformatics, [S.1.], v.7, p.511-523, 2010.

WU, L.; ZANG, J.; CHAN, R. Improved approach for haplotype inference based on Markov chain. In: LECTURE NOTES IN OPERATIONS RESEARCH. Anais... [S.l.: s.n.], 2008. p.204-215.

$\mathrm{XU}, \mathrm{H}$. et al. Comparison of haplotype inference methods using genotypic data from unrelated individuals. human heredity, [S.1.], v.2, n.58, p.63-68, 2004.

XU, H.-T. et al. The Effect of Mutations at Position E138 in HIV-1 Reverse Transcriptase and their Interactions with the M184I Mutation in Defining Patterns of Resistance to the Non-Nucleoside Reverse Transcriptase Inhibitors Rilpivirine and Etravirine. Antimicrob Agents Chemother, [S.1.], 2013. 
ZAKARIA, Z.; ISA, N.; SUANDI, S. A Study on Neural Network Training Algorithm for Multiface Detection in Static Images. International Conference on Computer, Electrical, Systems Science and Engineering. In: INTERNATIONAL CONFERENCE ON COMPUTER, ELECTRICAL, SYSTEMS SCIENCE AND ENGINEERING. Anais... [S.1.: s.n.], 2010. v.62, p.170-173.

ZAZZI, M. et al. Prediction of response to antiretroviral therapy by human experts and by the EuResist data-driven expert system (the EVE study). HIV Med, [S.1.], v.12, n.4, p.211-8, 2011.

ZAZZI, M. et al. Predicting response to antiretroviral treatment by machine learning: the euresist project. Intervirology, [S.1.], v.55, n.2, p.123-7, 2012.

ZHANG, J.-H. et al. A fast haplotype inference method for large population genotype data. Computational Statistics and Data Analysis, [S.1.], v.52, p.4891-4902, 2008. 